\title{
LOOP SPACES AND CONNECTIONS
}

\author{
DAVID BEN-ZVI AND DAVID NADLER
}

\begin{abstract}
We examine the geometry of loop spaces in derived algebraic geometry and extend in several directions the well known connection between rotation of loops and the de Rham differential. Our main result, a categorification of the geometric description of cyclic homology, relates $S^{1}$-equivariant quasicoherent sheaves on the loop space of a smooth scheme or geometric stack $X$ in characteristic zero with sheaves on $X$ with flat connection, or equivalently $\mathcal{D}_{X}$-modules. By deducing the Hodge filtration on de Rham modules from the formality of cochains on the circle, we are able to recover $\mathcal{D}_{X}$-modules precisely rather than a periodic version. More generally, we consider the rotated Hopf fibration $\Omega S^{3} \rightarrow \Omega S^{2} \rightarrow S^{1}$, and relate $\Omega S^{2}$-equivariant sheaves on the loop space with sheaves on $X$ with arbitrary connection, with curvature given by their $\Omega S^{3}$-equivariance.
\end{abstract}

\section{Contents}

1. Introduction

1.1. Loop spaces and odd tangent bundles

1.2. Setting of derived algebraic geometry

1.3. Loops in derived schemes

1.4. Rotating loops and the de Rham differential

1.5. Sheaves on loop spaces

1.6. Koszul dual interpretation: relation to $\mathcal{D}$-modules 10

1.7. Interpretation: categorified cyclic homology 13

1.8. Loops in derived stacks 14

2. Preliminaries 18

2.1. $\infty$-categories 18

2.2. Derived stacks 19

2.3. Graded modules 21

3. Affinization 24

3.1. Background 24

3.2. Affine derived stacks 25

3.3. Affinization of groups $\quad 26$

3.4. Application: circle actions 28

3.5. Unwinding higher coherences $\quad 29$

4. Loops in derived schemes 31

4.1. Loops and tangents 31

4.2. Equivariant sheaves and the de Rham differential 33

4.3. Holomorphic curvature $\quad 39$

5. Koszul dual description $\quad 40$

5.1. Graded Koszul duality 40

5.2. Twisted $\mathcal{D}$-modules 42

6. Loops in geometric stacks 44

6.1. Loops, unipotent loops, and formal loops 44 
6.2. Descent for differential forms

6.3. Formal loops and odd tangents 48

6.4. Equivariant sheaves on formal loop spaces 50

References

\section{INTRODUCTION}

This is the first of a two paper series 1 It contains general results on loop spaces in derived algebraic geometry. It establishes the intimate relation between equivariant sheaves on the free loop space of a stack and sheaves with connection on the stack itself. One can view this as a categorification of the familiar relation from topology and algebra between equivariant homology of free loop spaces (cyclic homology) and topological cohomology of spaces (singular or de Rham cohomology). The second paper BN2 of the series contains an application of this theory to flag varieties of reductive groups. In particular, we deduce consequences for the representation theory of Hecke algebras and Lie groups.

There is a surprising aspect to the relation between the theory developed in this first paper and the applications undertaken in the second. It is well known that flat connections in the form of $\mathcal{D}$-modules play a central role in representation theory. We show here that arbitrary connections can be understood in terms of the geometry of so called "small loops", or more precisely, loops in the formal neighborhood of constant loops (for schemes, all loops are small, but the distinction becomes important for stacks). Thus it is not surprising that small loops provide an alternative language to discuss geometric constructions of representations. But what is striking is that "large loops" naturally arise in the dual Langlands parametrization of representations. For example, we will see in the second paper that the notion of loop space organizes much of the seeming cacophany of spaces parametrizing representations of Lie groups.

This paper is organized as follows. In the remainder of the Introduction, we summarize our results (roughly following the overall structure of the paper). In Section 2 , we review the basic notions of $\infty$-categories and derived algebraic geometry which provide the context of our arguments. In Section 3 we develop the derived analogue of affinization and study group actions on affine derived schemes. In Section 4, we study equivariant sheaves on loop spaces of schemes. In Section 5, we provide a Koszul dual description of the previous results. Finally, in Section [6] we generalize the preceding results to loop spaces of geometric stacks.

1.1. Loop spaces and odd tangent bundles. The free loop space $\mathcal{L} X=\operatorname{Map}\left(S^{1}, X\right)$ of a topological space $X$ comes equipped with many fascinating structures. Perhaps the most fundamental is the $S^{1}$-action by loop rotation which recovers the constant loops $X \subset \mathcal{L} X$ as its fixed points. In this setting, the theory of equivariant localization for $S^{1}$-actions, relating the topology of a space with $S^{1}$-action to that of its fixed points, has been applied to spectacular effect by Witten and many others. Our aim is to contribute a categorified variation on this theme in the setting of sheaves in algebraic geometry.

To approach our results, let's first review some well known facts about functions and $S^{1}$ equivariant functions on loop spaces (for which an excellent reference is [LO], see also references therein). The universal paradigm is that Hochschild homology and cyclic homology of functions on a space provide a purely algebraic or categorical approach to functions and $S^{1}$-invariant functions respectively on its free loop space. For example, for a topological space $X$, Hochschild homology and cyclic homology of cochains on $X$ calculate the cohomology and $S^{1}$-equivariant

\footnotetext{
${ }^{1}$ This paper is a much expanded version of half of the preprint BN1].
} 
cohomology of $\mathcal{L} X[\mathrm{~J}]$. When applied to a commutative ring or more generally a scheme $X$, Hochschild homology gives the Dolbeault cohomology of differential forms on $X$ while cyclic homology gives the de Rham cohomology of $X$. Thus we can view the de Rham cohomology of a scheme $X$ as a realization of the $S^{1}$-invariant functions on some kind of free loop space of $X$.

The above interpretation of de Rham cohomology is quite familiar in the $\mathbf{Z} / 2 \mathbf{Z}$-graded supergeometry of mathematical physics. Namely, consider the complex cohomology of the circle $H^{*}\left(S^{1}, \mathbf{C}\right) \simeq \mathbf{C}[\eta] /\left(\eta^{2}\right)$, with $\eta$ of degree 1 , as the $\mathbf{Z} / 2 \mathbf{Z}$-graded supercommutative ring of functions on the odd line $\mathbf{C}^{0 \mid 1}$. For a smooth manifold $X$, the mapping space $\operatorname{Map}\left(\mathbf{C}^{0 \mid 1}, X\right)$ is the odd tangent supermanifold $\mathbb{T}_{X}[-1]$ with functions the $\mathbf{Z} / 2 \mathbf{Z}$-graded algebra of differential forms $\Omega_{X}^{-\bullet}=\operatorname{Sym}_{\mathcal{C}_{X}^{\infty}}^{\bullet}\left(\Omega_{X}[1]\right)$. Thus we see that $\mathbb{T}_{X}[-1]$ is a linear analogue of the free loop space: it parametrizes maps from a linear analogue of the circle, and its functions are the Hochschild homology of smooth functions on $X$. Furthermore, the linear analogue of the $S^{1}$-action of loop rotation is translation along the odd line $\mathbf{C}^{0 \mid 1}$. The de Rham differential $d$, thought of as an odd, square zero vector field on $\mathbb{T}_{X}[-1]$, is an infinitesimal generator of this action. Thus we see that the linear analogue of $S^{1}$-equivariant functions on the loop space is the cyclic homology of functions on $X$ in the form of its de Rham complex.

Similarly, in Z-graded super-algebraic geometry, we can consider the spectrum of the cohomology $H^{*}\left(S^{1}, \mathbf{C}\right) \simeq \mathbf{C}[\eta] /\left(\eta^{2}\right)$, with $\eta$ of degree 1 . Note that the cohomology is the free supercommutative $\mathbf{C}$-algebra on $\eta$, and so its spectrum can be thought of as a shifted version $\mathbb{A}^{1}[1]$ of the affine line. In other words, the cohomology is the $\mathbf{Z}$-graded supercommutative ring of functions on an infinitesimal of degree -1 . To emphasize the group structure on $\mathbb{A}^{1}[1]$, we will also often refer to it as the classifying stack $B \mathbb{G}_{a}$ (just as one uses the alternative notation $\mathbb{G}_{a}$ to emphasize the group structure on the affine line $\mathbb{A}^{1}$ ). For a smooth scheme $X$, the mapping space $\operatorname{Map}\left(B \mathbb{G}_{a}, X\right)$ is the shifted tangent bundle $\mathbb{T}_{X}[-1]$ with functions differential forms $\Omega_{X}^{-\bullet}=\operatorname{Sym}_{\mathcal{O}_{X}}^{\bullet}\left(\Omega_{X}[1]\right)$ placed in negative (or homological) degrees, and the linear analogue of loop rotation is again the degree -1 , square zero vector field given by the de Rham differential $d$.

In algebraic geometry, there is another more traditional path to the relation between free loop spaces and shifted tangent bundles. Let's model the circle as a simplicial complex with two points connected by two line segments, and try to interpret concretely what maps from such an object to a smooth scheme $X$ should be. First, mapping two points to $X$ defines the product $X \times X$. One of the line segments connecting the points says the points are equal: we should impose the equation $x=y$ that defines the diagonal $\Delta \subset X \times X$. Then the other line segment says the points are equal again, so we should impose the equation $x=y$ again, or in other words, take the self-intersection $\Delta \cap \Delta \subset X \times X$. Of course, we could interpret this naively as being a copy of $X$ again, however the intersection is far from transverse: indeed the tensor product

$$
\mathcal{O}_{\Delta \cap \Delta}=\mathcal{O}_{\Delta} \otimes_{\mathcal{O}_{X \times X}} \mathcal{O}_{\Delta}
$$

needs to be derived since there are higher Tor terms. The derived version of this tensor product is in fact the definition of the Hochschild homology of $\mathcal{O}_{X}$. We can use the Koszul complex to arrive at the homological Hochschild-Kostant-Rosenberg isomorphism

$$
\mathcal{O}_{\Delta} \otimes_{\mathcal{O}_{X \times X}}^{\mathbb{L}} \mathcal{O}_{\Delta} \simeq \Omega_{X}^{-\bullet} \text {. }
$$

Thus we again find that the shifted tangent bundle $\mathbb{T}_{X}[-1]$ whose functions are differential forms $\Omega_{X}^{-\bullet}$ plays the role of the free loop space of $X$.

1.2. Setting of derived algebraic geometry. We would like to generalize the ideas in the above discussion to a setting for algebraic geometry where the target $X$ could not only be a scheme but also perhaps a scheme with symmetry in the form of a stack. Thus we seek a 
theoretical framework that encompasses both stacks and the sophisticated derived intersections involved in calculating loop spaces.

Derived algebraic geometry, whose foundations have been developed recently by Toën and Vezzosi [HAG1, HAG2] and Lurie [L1, L2, L3], provides a natural setting in which we can apply constructions from algebraic topology - such as homotopy colimits, homotopy limits, and mapping spaces - to the classical objects of algebraic geometry.

Derived algebraic geometry generalizes classical algebraic geometry simultaneously in two directions: it encompasses the theory of stacks, allowing for derived versions of quotients (and more general gluings or colimits) of schemes, and also the theory of differential graded schemes, allowing for derived versions of intersections (and more general equations or limits). In both cases, passing to the derived or corrected versions of the operation guarantees that no meaningful geometric information is lost. For example, if we were to work within the theory of stacks alone, the mapping object $\operatorname{Map}\left(S^{1}, X\right)$ would make sense, but it would only recover $X$ not its odd tangent bundle when $X$ is a smooth scheme.

In broad outline, to arrive at derived algebraic geometry from classical algebraic geometry, one takes the following steps.

First, one replaces commutative rings by a notion of derived commutative rings. There are many choices with different geometric and homotopical flavors, but for our applications we will work over a fixed ground $\mathbf{Q}$-algebra $k$. By a derived ring, we will mean a commutative differential graded $k$-algebra which is cohomologically trivial in positive degrees. In other words, a derived ring is a commutative ring object in non-positively graded cochain complexes over $k$. Following usual conventions, we will use the term affine derived scheme to mean an object of the opposite category of derived rings. One can view a general derived scheme as an ordinary scheme over $k$ equipped with a sheaf of derived rings whose zeroth cohomology is identified with the structure sheaf of the scheme, and whose other cohomology sheaves are quasicoherent. Introducing derived rings allows for derived intersections where we replace the tensor product of rings with its derived functor.

Second, one considers functors of points on derived rings that take values not only in sets but in simplicial sets or equivalently topological spaces. (For example, derived rings naturally give such functors via the Dold-Kan correspondence.) This allows for derived quotients by keeping track of gluings in the enriched theory of spaces. Roughly speaking, a derived stack is a functor from derived rings to topological spaces satisfying an appropriate sheaf axiom with respect to the étale topology on derived rings. Examples include all of the schemes of classical algebraic geometry, stacks of modern algebraic geometry, and topological spaces of homotopy theory (in the form of locally constant stacks). From this perspective, an affine derived scheme is simply a representable functor. Any derived stack has an "underlying" underived stack, obtained by restricting its functor of points to ordinary rings. In fact, derived stacks can be viewed as formal thickenings of underived stacks, just as supermanifolds are infinitesimal thickenings of manifolds.

One of the complicated aspects of the above theory is that the domain of derived rings, target of topological spaces, and functors of points themselves must be treated with the correct enriched homotopical understanding. We recommend Toën's excellent survey To2 for more details and references. It was our introduction to many of the notions of derived algebraic geometry, in particular derived loop spaces. Of particular relevance to this paper are the closed monoidal structure on derived stacks (the Cartesian product and mapping space functors on topological spaces induce analogous functors on derived stacks), and the theory of cotangent complexes for Artin derived stacks [HAG2, L2]. The reader will find in Section 2 a very brief 
synopsis of the theory of $\infty$-categories which provides perhaps the most succinct categorical foundations.

1.2.1. Free loop space. The homotopy theory of topological spaces naturally maps to the world of derived algebraic geometry via (the sheafification of) constant functors from derived rings to topological spaces. Any topological space defines a derived stack (in fact, with trivial derived structure) which we will refer to by the same name. So in particular, the integers $\mathbf{Z}$ define an abelian group stack, as does its classifying space the circle $S^{1}=B \mathbf{Z}$.

With the circle in hand, we define the free loop space of a derived stack $X$ to be the derived mapping stack

$$
\mathcal{L} X=\operatorname{Map}\left(S^{1}, X\right) .
$$

To $S$ a test derived ring, $\mathcal{L} X$ assigns the space of maps Spec $S \times S^{1} \rightarrow X$ between sheaves of topological spaces on the étale topology of derived rings.

It follows that for $X=\operatorname{Spec} R$ the spectrum of a derived ring, the loop space $\mathcal{L} X=$ $\operatorname{Map}\left(S^{1}, X\right)$ is equivalent to the spectrum of the Hochschild chain complex

$$
H C(R)=R \otimes S^{1}=R \otimes_{R \otimes R} R
$$

of the derived ring $R$. In the case that $R$ is an ordinary ring and regular, so that $X$ is an underived smooth affine scheme, we can use the Koszul resolution to calculate its loop space and arrive at the odd tangent bundle

$$
\mathcal{L} X \simeq \mathbb{T}_{X}[-1]=\operatorname{Spec}_{\operatorname{Sym}_{\mathcal{O}_{X}}}^{\bullet}\left(\Omega_{X}[1]\right)
$$

in agreement with our discussion above. In particular, $\mathcal{L} X$ is a derived thickening of $X$ itself. We will see below that this picture extends to arbitrary derived schemes.

On the other hand, for a stack $X$, it is easy to see that its loop space $\mathcal{L} X$ is a derived thickening of the inertia stack of $X$ parametrizing points of $X$ equipped with automorphisms. For example, the loop space $\mathcal{L}(B G)$ of the classifying space $B G=p t / G$ of a group is the quotient $G / G$ by the conjugation action. We discuss this example in more detail in Section 1.8 . In [BN2, we study in detail a collection of targets associated with quotients of flag varieties.

1.3. Loops in derived schemes. We first focus on loop spaces of derived schemes, and discuss generalizations to stacks below. By a derived scheme, we will always mean a quasi-compact derived scheme with affine diagonal over a base $\mathbf{Q}$-algebra $k$. The basic building blocks of derived algebraic geometry are affine derived schemes, and all of our results for schemes and stacks are built up from this base case via descent along gluing constructions.

We show that the identification of loop spaces with odd tangent bundles extends to arbitrary derived schemes, once we replace the tangent bundle with the tangent complex, as developed in the present context in [HAG2, 1.4] and [L2, 7.4] following Quillen and Illusie. The tangent complex $\mathbb{T}_{X}$ is the natural derived analogue of the tangent bundle, for example in the sense that $\mathbb{T}_{X}$ can be identified with the derived mapping stack from the dual numbers Spec $k[\epsilon] /\left(\epsilon^{2}\right)$ to the derived stack $X$. The tangent complex of an affine derived scheme is concentrated in non-negative degrees, degrees) while the tangent complex of an underived smooth stack lies in non-positive degrees.

We will take repeated advantage of the notion of affinization in the sense of Toën To1 (reviewed and extended in Section (3). The affinization $\operatorname{Aff}(X)$ of a derived stack $X$ is defined by the universal property that any map $X \rightarrow$ Spec $R$ to an affine derived scheme factors through a canonical map $X \rightarrow \operatorname{Aff}(X)$. The affinization of a topological space $S$ over a ground ring $k$ captures the topology of $S$ seen by its cochains over $k$. In particular, since we take $k$ to be a Q-algebra, this construction is (the $k$-linear) part of the rational homotopy theory of $S$. (See To1] for applications of affinization to the algebro-geometric study of homotopy types.) 
In the case of $S^{1}=B \mathbf{Z}$, the affinization map is given by the canonical map

$$
S^{1}=B \mathbf{Z} \longrightarrow B \mathbb{G}_{a}
$$

induced by the canonical map from $\mathbf{Z}$ to the affine line $\mathbb{A}^{1} \simeq \mathbb{G}_{a}$ (we use the notation $\mathbb{G}_{a}$ to emphasize the group structure on the affine line). In other words, the induced pullback on functions

$$
k[\eta] /\left(\eta^{2}\right)=\mathcal{O}\left(B \mathbb{G}_{a}\right) \longrightarrow \mathcal{O}\left(S^{1}\right) \simeq H^{*}\left(S^{1}, k\right), \quad \text { with } \eta \text { of degree } 1,
$$

is an equivalence.

It is easy to see that loop spaces are local objects in the Zariski topology (see Lemma4.2). As a result, many results on loops in affine derived schemes carry over without change to arbitrary derived schemes (which we always assume to have affine diagonal). (On the other hand, loops are not local in the étale topology. We will turn to the more complicated situation of loops in stacks in Section 1.8 of the introduction.) Hence as a consequence of affinization, we are able to establish (as Proposition 4.4) the following form of the homological Hochschild-KostantRosenberg theorem for loops in derived schemes.

Proposition 1.1. Fix a derived scheme $X$ over $k$, and consider its cotangent complex $\Omega_{X}$.

(1) The mapping derived stack $\operatorname{Map}\left(B \mathbb{G}_{a}, X\right)$ is equivalent to the odd tangent bundle $\mathbb{T}_{X}[-1]=$ $\operatorname{Spec} \operatorname{Sym}_{\mathcal{O}_{X}}\left(\Omega_{X}[1]\right)$.

(2) The affinization map $S^{1} \rightarrow B \mathbb{G}_{a}$ induces an equivalence

$$
\operatorname{Map}\left(B \mathbb{G}_{a}, X\right)=\mathbb{T}_{X}[-1] \stackrel{\sim}{\longrightarrow} \mathcal{L} X=\operatorname{Map}\left(S^{1}, X\right) .
$$

Thus we have a canonical Hochschild-Kostant-Rosenberg equivalence for Hochschild chains

$$
H C\left(\mathcal{O}_{X}\right) \simeq \operatorname{Sym}_{\mathcal{O}_{X}}\left(\Omega_{X}[1]\right)
$$

1.4. Rotating loops and the de Rham differential. Next we turn to the $S^{1}$-action on the loop space $\mathcal{L} X=\operatorname{Map}\left(S^{1}, X\right)$ of a derived scheme by loop rotations, or in other words, the action induced by the regular $S^{1}$-action on the domain $S^{1}$.

Let us first discuss the general notion of group actions on stacks. The action of a group derived stack $G$ on a derived stack $X$ involves an action map $G \times X \rightarrow X$ together with associativity and higher coherences. When $G=S^{1}$, the identification $S^{1}=B \mathbf{Z}$ implies the action map $S^{1} \times X \rightarrow X$ is the same as an automorphism (or self-homotopy) of the identity map of $X$. When $X$ is a derived scheme and $G=B \mathbb{G}_{a}$, the action map $B \mathbb{G}_{a} \times X \rightarrow X$ is the same as a section of the odd tangent bundle $\mathbb{T}_{X}[-1]$, or in other words, a vector field of degree -1 . The associativity constraint for a $B \mathbb{G}_{a}$-action is equivalent to requiring the square of this vector field to be homotopic to zero. In general, there are infinitely many higher coherences that need to be specified to give an action. We will see below that in the case of loop spaces, all of the higher coherences are encoded in these initial data.

As another consequence of affinization, we are able to identify $S^{1}$-actions on affine derived schemes with $B \mathbb{G}_{a}$-actions (see Corollary 3.15 below, or ToVe5] for a model categorical version). The result can be viewed as a nonlinear analogue of the classical identification (see Kas, Lo, DK, or Corollary 3.14 below) between $k$-modules with an $S^{1}$-action (cyclic modules) and $k$ modules with a square zero endomorphism of degree -1 (mixed complexes). When we apply this to the canonical $S^{1}$-action on the loop space $\mathcal{L} X$ of a derived scheme, we obtain the following (as Proposition 4.7).

Proposition 1.2. Let $X$ be a derived scheme over $k$. Under the identification $\mathcal{L} X \simeq \mathbb{T}_{X}[-1]$, the rotation $S^{1}$-action on $\mathcal{L} X$ corresponds to the translation $B \mathbb{G}_{a}$-action on $\mathbb{T}_{X}[-1]$. In turn, 
the vector field of degree -1 coming from the $B \mathbb{G}_{a}$-action on $\mathbb{T}_{X}[-1]$ is given by the de Rham differential.

The natural $\mathbb{G}_{m}$-action on $\mathbb{G}_{a}$, and hence $B \mathbb{G}_{a}$, induces a corresponding $\mathbb{G}_{m}$-action on the mapping space $\operatorname{Map}\left(B \mathbb{G}_{a}, X\right)$, and thus on the loop space $\mathcal{L} X$. From the perspective of the affinization identification $B \mathbb{G}_{a} \simeq \operatorname{Aff}\left(S^{1}\right)$, the $\mathbb{G}_{m}$-action on $B \mathbb{G}_{a}$ results from the formality $\mathcal{O}\left(S^{1}\right) \simeq H^{*}\left(S^{1}, k\right)$. Under the identification $\mathbb{T}_{X}[-1] \simeq \operatorname{Map}\left(B \mathbb{G}_{a}, X\right)$, the $\mathbb{G}_{m}$-action on $\mathbb{T}_{X}[-1]$ coincides with the natural dilation $\mathbb{G}_{m}$-action. Equivalently, it recovers the grading on the algebra $\operatorname{Sym}_{\mathcal{O}_{X}}\left(\Omega_{X}[1]\right)$, and hence the Hodge filtration on the de Rham complex. Keeping track of this $\mathbb{G}_{m}$-action leads to formality statements that significantly simplify the equivariant geometry of loop spaces. In particular, we deduce the following uniqueness statement in Section 4.2.1 from an examination of the dilation $\mathbb{G}_{m}$-weights on $\Omega_{X}^{-\bullet}$ (see also Remark 1.9).

Theorem 1.3. For $X$ a smooth scheme over $k$, the tautological $B \mathbb{G}_{a}$-action on $\mathbb{T}_{X}[-1]=$ $\operatorname{Map}\left(B \mathbb{G}_{a}, X\right)$ is the unique such action (up to equivalence) extending the de Rham differential and compatible with $\mathbb{G}_{m}$-actions.

Remark 1.4. Let us remind the reader that local notions of morphisms of schemes naturally extend to derived schemes in the following way. Given a map of derived rings $R \rightarrow S$, one can ask that the induced map of discrete rings $\pi_{0}(R) \rightarrow \pi_{0}(S)$ satisfies the property of interest, and that the higher homotopy groups of $S$ are simply base changes of those of $R$ along the morphism $\pi_{0}(R) \rightarrow \pi_{0}(S)$.

As a special case, a derived scheme $X \rightarrow \operatorname{Spec} k$ is smooth if and only if it is a smooth underived scheme.

1.5. Sheaves on loop spaces. Now we will consider the interaction of loop rotation with quasicoherent sheaves on loop spaces. Quasicoherent sheaves on a derived stack $X$ form a stable $\infty$-category defined as follows.

For $X=\operatorname{Spec} R$ an affine derived scheme, we set $\mathrm{QC}(X)=\operatorname{Mod}_{R}$, the $\infty$-category of $R$ modules. Objects of $\operatorname{Mod}_{R}$ are differential graded modules over the differential graded $k$-algebra $R$, and morphisms are module maps localized with respect to quasi-isomorphisms. Given a map $f: \operatorname{Spec} S \rightarrow \operatorname{Spec} R$, the pullback $f^{*}: \mathrm{QC}(\operatorname{Spec} R) \rightarrow \mathrm{QC}(\operatorname{Spec} S)$ is the usual base change functor $f^{*}(M)=M \otimes_{R} S$.

We can tautologically write any derived stack as a colimit of affine derived schemes, and then extend the above assignment to a colimit-preserving functor QC from the $\infty$-category of derived stacks to the opposite of the $\infty$-category of stable $\infty$-categories. So informally speaking, a quasicoherent sheaf on a derived stack $X$ is a compatible collection of modules on all affine derived schemes over $X$.

1.5.1. $S^{1}$-equivariant sheaves. An $S^{1}$-action on a derived stack $Z$ induces an automorphism of the identity functor of $\mathrm{QC}(Z)$. For any quasicoherent sheaf $\mathcal{F} \in \mathrm{QC}(Z)$, the resulting automorphism of $\mathcal{F}$ is given by the monodromy of $\mathcal{F}$ along the $S^{1}$-orbits. To equip $\mathcal{F}$ with an $S^{1}$-equivariant structure is to trivialize this automorphism, or equivalently, to kill the variation (the difference between the automorphism and the identity). In what follows, we write $\mathrm{QC}(X)^{S^{1}}$ for the stable $\infty$-category of equivariant quasicoherent sheaves.

In the case of the loop space $\mathcal{L} X \simeq \mathbb{T}_{X}[-1]$ of a derived scheme $X$, its functions are the de Rham algebra $\Omega_{X}^{-\bullet}=\operatorname{Sym}_{\mathcal{O}_{X}}^{\bullet}\left(\Omega_{X}[1]\right)$, and loop rotation corresponds to the vector field of degree -1 given by the de Rham differential. The following theorem demonstrates that in the smooth and underived setting, these data fully account for $S^{1}$-equivariant sheaves. Throughout what follows, we keep track of the additional natural $\mathbb{G}_{m}$-weight grading to that $\Omega_{X}^{-\bullet}$ sits along the 
diagonal in the cohomological degree and $\mathbb{G}_{m}$-weight bigrading. In particular, the generating one-forms $\Omega_{X}[1] \subset \Omega_{X}^{-\bullet}$ sit in cohomological degree -1 and $\mathbb{G}_{m}$-weight grading -1 .

Let $\Omega_{X}^{-\bullet}[d]$ be the $\mathbf{Z}$-graded sheaf of differential graded $k$-algebras on $X$ obtained from the de Rham algebra by adjoining the de Rham differential in cohomological degree -1 and $\mathbb{G}_{m}$-weight grading -1 , so that $[d, \omega]=d \omega$, for $\omega \in \Omega_{X}^{-\bullet}$, and $d^{2}=0$. We write $\Omega_{X}^{-\bullet}[d]$-mod for the stable $\infty$-category of quasicoherent sheaves on $X$ equipped with a compatible structure of differential graded $\Omega_{X}^{-} \cdot[d]$-module. We also append the subscript $\mathbf{Z}$ to the notation to denote differential graded modules with an additional compatible $\mathbb{G}_{m}$-weight grading.

Theorem 1.5. For a smooth scheme $X$ over $k$, there are canonical equivalences

$$
\begin{gathered}
\mathrm{QC}(\mathcal{L} X)^{S^{1}} \simeq \Omega_{X}^{-\bullet}[d]-\bmod \\
\mathrm{QC}(\mathcal{L} X)^{B \mathbb{G}_{a} \rtimes \mathbb{G}_{m}} \simeq \Omega_{X}^{-\bullet}[d]-\bmod _{\mathbf{z}}
\end{gathered}
$$

Remark 1.6. The finiteness and formality of the cochain algebra $C^{*}\left(S^{1}\right)$ are the primary technical inputs to the theorem.

Remark 1.7. For $X$ an arbitrary derived scheme with affine diagonal, we obtain a version of Theorem 1.5 describing $S^{1}$-equivariant sheaves on $\mathcal{L} X$ as modules over $\Omega_{X}^{-\bullet}$ adjoined chains on $S^{1}$, whose action is related via Proposition 1.2 with the de Rham differential. In the absence of a more naive description, such as given by the formality of Theorem 1.3, we may interpret this as a definition of de Rham modules on $X$.

Remark 1.8. Later in the introduction, we will interpret the graded equivalence of the theorem as providing the Hodge filtration on the $\infty$-category of de Rham modules on $X$.

Remark 1.9. Theorem 1.5 and the results preceding it are sketched in the preprint BN1. An alternative approach to the necessary foundations, in particular the formality of Theorem 1.3 (in fact, a model categorical refinement thereof) is developed in the recent work ToVe5, and applied in [ToVe4].

In differential geometry, there is a familiar relationship between quasicoherent sheaves $\mathcal{E}$ with flat connection $\nabla$ on $X$ and modules for $\Omega_{X}^{-\bullet}[d]$. Namely, the connection $\nabla: \mathcal{E} \rightarrow \mathcal{E} \otimes \Omega_{X}$ naturally extends to an $\Omega_{X}^{-\bullet}[d]$-module structure on the pullback

$$
\pi^{*} \mathcal{E}=\mathcal{E} \otimes_{\mathcal{O}_{X}} \Omega_{X}^{-\bullet}
$$

The action of the distinguished element $d$ is given by the formula

$$
d(\sigma \otimes \omega)=\nabla(\sigma) \wedge \omega+\sigma \otimes d \omega, \quad \sigma \otimes \omega \in \mathcal{E} \otimes_{\mathcal{O}_{X}} \Omega_{X}^{-\bullet} .
$$

The fact that $\nabla$ is flat leads directly to the identity $d^{2}=0$. To further refine this to an equivalence, we will consider a suitable form of Koszul duality below.

1.5.2. Hopf fibration. We now examine what happens when we replace equivariance for circle actions by a rougher notion of invariance under rotation. In the case of sheaves on loop spaces, we will find that this precisely corresponds to allowing curvature.

Recall that the action of a monoid $G$, with identity $e \in G$, on a scheme or stack $X$ consists of a pointed action map $G \times X \rightarrow X$, so that $\{e\} \times X \rightarrow X$ is homotopic to the identity, together with an associativity constraint and a collection of higher coherences. We can begin to unwind this data by first considering what information is contained in the pointed action map alone. When we take $G$ to be the circle $S^{1}$ or its affinization $B \mathbb{G}_{a}$, we are asking to what extent we can understand actions from automorphisms of the identity or odd vector fields respectively. 
For any pointed derived stack $Y_{*}$, we can apply a variant of the James construction in topology and introduce the free monoid on $Y_{*}$ via based loops in the suspension

$$
F\left(Y_{*}\right)=\Omega \Sigma Y_{*} .
$$

Then a pointed map $Y_{*} \times X \rightarrow X$ is equivalent to an action of the monoid $F\left(Y_{*}\right)$ on $X$. If $Y_{*}$ itself has a monoid structure then we also have a canonical homomorphism $F\left(Y_{*}\right) \rightarrow Y_{*}$, and we can measure obstructions for an $F\left(Y_{*}\right)$-action to descend to a $Y_{*}$-action.

Now let's focus on the case $Y_{*}=S^{1}$ pointed by the identity so that

$$
F\left(S^{1}\right) \simeq \Omega \Sigma S^{1} \simeq \Omega S^{2} .
$$

The canonical map $\Omega S^{2} \rightarrow S^{1}$ determined by the monoidal structure on $S^{1}$ is given by applying $\Omega$ to the standard inclusion

$$
S^{2} \simeq \mathbf{C P}^{1} \longrightarrow B S^{1} \simeq \mathbf{C P}^{\infty} .
$$

In turn, this map is the classifying map for the Hopf fibration

$$
S^{1} \longrightarrow S^{3} \longrightarrow S^{2} \text {. }
$$

Now we have the following variation on Theorem 1.5. In fact, it is a simpler homotopical statement and our arguments do not require that $X$ be smooth or underived. In agreement with our previous conventions, we will work with the cohomology

$$
\mathcal{O}\left(S^{2}\right) \simeq C^{*}\left(S^{2}, k\right) \simeq H^{*}\left(S^{2}, k\right) \simeq k[u] /\left(u^{2}\right)
$$

as a graded algebra with $u$ in cohomological degree 2 and $\mathbb{G}_{m}$-weight grading 1 . Consider the $\mathbf{Z}$-graded sheaf of differential graded $k$-algebras on $X$ given by the tensor product

$$
\Omega_{X, S^{2}}^{-\bullet}=\Omega_{X}^{-\bullet} \otimes \mathcal{O}\left(S^{2}\right) \simeq \Omega_{X}^{-\bullet}[u] /\left(u^{2}\right)
$$

with differential

$$
\delta(\omega \otimes 1)=d \omega \otimes u \quad \delta(\omega \otimes u)=0
$$

where as usual $d$ denotes the de Rham differential. One arrives at $\Omega_{X, S^{2}}^{-\bullet}$ by taking functions along the fibers of the map

$$
\mathcal{L} X / \Omega S^{2} \longrightarrow S^{2} \longrightarrow p t .
$$

Our particular presentation comes from thinking of the $E_{2}$-page of the spectral sequence for the first map in the above sequence.

We write $\Omega_{X, S^{2}}^{-\bullet}$ mod for the stable $\infty$-category of quasicoherent sheaves on $X$ equipped with a compatible structure of differential graded $\Omega_{X, S^{2}}^{-\bullet}$-module. As before, we also append the subscript $\mathbf{Z}$ to the notation to denote differential graded modules with an additional compatible weight grading.

Theorem 1.10. For a derived scheme $X$ over $k$, there are canonical equivalences

$$
\begin{gathered}
\mathrm{QC}(\mathcal{L} X)^{\Omega S^{2}} \simeq \Omega_{X, S^{2}}^{-\bullet} \text { mod } \\
\mathrm{QC}(\mathcal{L} X)^{\operatorname{Aff}\left(\Omega S^{2}\right) \rtimes \mathbb{G}_{m}} \simeq \Omega_{X, S^{2}}^{-\bullet}-\bmod _{\mathbf{z}}
\end{gathered}
$$

Remark 1.11. The finiteness and formality of the cochain algebra $C^{*}\left(S^{2}\right)$ are the primary technical inputs to the theorem.

We will interpret the theorem in terms of sheaves with not necessarily flat connection in the section immediately following. 
1.6. Koszul dual interpretation: relation to $\mathcal{D}$-modules. Throughout the discussion here, we assume that $X$ is a smooth underived scheme.

As discussed above, modules over the de Rham complex are intimately related to sheaves with flat connection or $\mathcal{D}$-modules. In fact, as explained by [Ka, $\mathrm{BD}$, there is a natural Koszul equivalence between appropriate derived categories of modules over the de Rham complex and $\mathcal{D}$-modules. This is a noncommutative version of the familiar pattern relating sheaves on an odd tangent bundle (modules over an exterior algebra) and sheaves on an even cotangent bundle (modules over a symmetric algebra). More specifically, it is a case of the nonhomogeneous quadratic duality studied in $[\mathrm{P}, \mathrm{PP}$, which exchanges differential graded quadratic algebras (in particular, the de Rham complex and its curved versions) and filtered quadratic algebras. In our setting, Koszul duality arises from $S^{1}$-equivariance, as in [GKM]: the exterior algebra arises as the chains on $S^{1}$, and its Koszul dual as the cochains on $B S^{1}$.

In Section 2.3 below, we discuss the general yoga of categories of graded modules over graded algebras and their independence of shears of grading, which accounts for the different conventions of [BGS] and GKM], as well as the notion of periodic localization (as it appears in cyclic homology theory). We apply these notions in Section 5, where we discuss Koszul duality for de Rham modules. In our setting, the Koszul dual to the de Rham algebra $\Omega_{X}^{-\bullet}=\operatorname{Sym}_{\mathcal{O}_{X}}\left(\Omega_{X}[1]\right)$ is the bigraded symmetric algebra $\operatorname{Sym}_{\mathcal{O}_{X}}\left(\mathbb{T}_{X}[-2]\right)$. Turning on the the de Rham differential is Koszul dual to a noncommutative deformation: the Koszul dual to the extended algebra $\Omega_{X}^{-\bullet}[d]$ is the corresponding cohomologically graded Rees algebra $\mathcal{R}_{X}$ built out of the canonical filtration of the algebra of differential operators. Furthermore, the algebras have natural additional weight gradings coming from dilations, and are Koszul dual as bigraded algebras. Thus appropriate derived categories of graded modules over the algebras are equivalent.

Remark 1.12. Any complications in the statements below stem from the familiar issue in Koszul duality that one must impose some finiteness on sheaves to obtain equivalences of categories. Geometrically speaking, we need to impose such conditions in order for the augmentation section to see all of the structure of the sheaves.

1.6.1. Flat modules. First, we can complete the cotangent bundle along the zero section, and consider complete modules over the corresponding complete graded Rees algebra $\widehat{\mathcal{R}}_{X}$. We write $\widehat{\mathcal{R}}_{X}$-cmod for the stable $\infty$-category of quasicoherent sheaves on $X$ equipped with the structure of complete $\widehat{\mathcal{R}}_{X}$-module. The following results from Theorem 1.5 by considering the natural

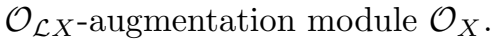

Corollary 1.13. For a smooth underived scheme $X$, there are canonical equivalences of stable $\infty$-categories

$$
\begin{gathered}
\mathrm{QC}(\mathcal{L} X)^{B \mathbb{G}_{a} \rtimes \mathbb{G}_{m}} \simeq \widehat{\mathcal{R}}_{X-\operatorname{cmod}} \mathbf{z} \\
\mathrm{QC}(\mathcal{L} X)^{B \mathbb{G}_{a}} \simeq \widehat{\mathcal{R}}_{X}-\operatorname{cmod}
\end{gathered}
$$

Alternatively, we can restrict to stable $\infty$-categories of suitably finite modules. Let us write $\operatorname{Perf}_{X}(\mathcal{L} X)$ for the small, stable $\infty$-subcategory of $\mathrm{QC}(\mathcal{L} X)$ of quasicoherent sheaves whose pushforward along the canonical map $\mathcal{L} X \rightarrow X$ are perfect. Note that the structure sheaf $\mathcal{O}_{\mathcal{L} X} \simeq \operatorname{Sym}_{\mathcal{O}_{X}}^{\bullet}\left(\Omega_{X}[1]\right)$ is perfect over $\mathcal{O}_{X}$, and the $\mathcal{O}_{\mathcal{L} X}$-augmentation module $\mathcal{O}_{X}$ is obviously perfect over $\mathcal{O}_{X}$ (though not over $\mathcal{O}_{\mathcal{L} X}$ ). We write $\mathcal{R}_{X}$-perf for the stable $\infty$-category of quasicoherent sheaves on $X$ equipped with a compatible structure of perfect $\mathcal{R}_{X}$-module.

Corollary 1.14. For a smooth underived scheme $X$, there are canonical equivalences of small, stable $\infty$-categories

$$
\begin{gathered}
\operatorname{Perf}_{X}(\mathcal{L} X)^{B \mathbb{G}_{a} \rtimes \mathbb{G}_{m}} \simeq \mathcal{R}_{X} \text {-perf } \\
\operatorname{Perf}_{X}(\mathcal{L} X)^{B \mathbb{G}_{a}} \simeq \mathcal{R}_{X} \text {-perf. }
\end{gathered}
$$


1.6.2. Periodic localization. To recover $\mathcal{D}_{X}$-modules from $\mathcal{R}_{X}$-modules, we must pass to periodic modules in the equivalence of Corollary 1.14. All of the categories are compatibly linear over the ring $H^{*}\left(B S^{1}\right) \simeq k[u]$, with $u$ of cohomological degree 2 and $\mathbb{G}_{m}$-weight grading 1 . On the one hand, any category of $S^{1}$ or $B \mathbb{G}_{a}$-equivariant sheaves is linear over the equivariant cohomology ring $\mathcal{O}\left(B \mathbb{G}_{a}\right) \simeq H^{*}\left(B S^{1}\right)$. On the other hand, by construction, $k[u]$ maps to the graded Rees algebra $\mathcal{R}_{X}$ as a central subalgebra.

When we invert $u$ and localize the categories, we will denote the result by the subscript per. In general, since $u$ is of the cohomological degree 2 , a periodic category is only $\mathbf{Z} / 2 \mathbf{Z}$-graded. But when we localize a mixed category compatibly graded by $\mathbb{G}_{m}$-weight, the periodic category maintains a usual cohomological degree. Using the shear equivalence of Section 2.3, we find that the $\infty$-category of $B \mathbb{G}_{a} \rtimes \mathbb{G}_{m}$-equivariant sheaves on the loop space naturally has the structure of $\mathbb{G}_{m}$-equivariant category over the ungraded line $\mathbb{A}^{1}$. We can then simply realize periodic localization as restriction to the open $\mathbb{G}_{m}$-orbit $\mathbb{G}_{m} \subset \mathbb{A}^{1}$.

Corollary 1.15. There are canonical equivalences of small, stable $\infty$-categories

$$
\begin{gathered}
\operatorname{Perf}_{X}(\mathcal{L} X)_{\text {per }}^{B \mathbb{G}_{a} \rtimes \mathbb{G}_{m}} \simeq \mathcal{D}_{X} \text {-perf. } \\
\operatorname{Perf}_{X}(\mathcal{L} X)_{\text {per }}^{B \mathbb{G}_{a}} \simeq \mathcal{D}_{X} \text {-perf } \otimes_{k} k\left[u, u^{-1}\right] .
\end{gathered}
$$

The second equivalence is of $\mathbf{Z} / 2 \mathbf{Z}$-periodic stable $\infty$-categories.

Thus we think of loop spaces and their circle action as a useful geometric counterpart to cotangent bundles and their quantization. Our original motivation for this story came from the observation that in applications to representation theory, it is often easier to identify the de Rham differential rather than the ring of differential operators.

1.6.3. The Hodge filtration. As we discussed above, the $\infty$-category $\mathrm{QC}(\mathcal{L} X)^{B \mathbb{G}_{a} \rtimes \mathbb{G}_{m}}$ of graded de Rham modules naturally localizes over the stack $\mathbb{A}^{1} / \mathbb{G}_{m}$, where the coordinate on the line is identified (after shear of grading) with the generator for the cohomology of $B S^{1}$. Recall that by the general yoga of the Rees construction, objects over $\mathbb{A}^{1} / \mathbb{G}_{m}$ should be thought of as filtrations on their restrictions to $p t=\mathbb{G}_{m} / \mathbb{G}_{m} \subset \mathbb{A}^{1} / \mathbb{G}_{m}$. In particular, the Hodge filtration on nonabelian de Rham cohomology of a scheme $X$ was defined by Simpson $[\underline{\mathrm{S}}$ ] in this fashion, replacing local systems or $\mathcal{D}_{X}$-modules by their filtered versions as Rees modules. (See also [ST] for the related development of the general theory of $\mathcal{D}$-modules on stacks.) The Koszul duality of de Rham modules and Rees modules above identifies Simpson's Hodge filtration with our filtration on de Rham modules. We thus are led to the following interpretation of our results:

Corollary 1.16. The $\infty$-category $\operatorname{Perf}_{X}(\mathcal{L} X)^{B \mathbb{G}_{a} \rtimes \mathbb{G}_{m}}$ over $\mathbb{A}^{1} / \mathbb{G}_{m}$ coincides with Simpson's Hodge filtration on nonabelian de Rham cohomology (specifically, of the $\infty$-category of perfect $\mathcal{D}_{X}$-modules).

1.6.4. Curved modules. We have seen that $S^{1}$-equivariant sheaves on the loop space $\mathcal{L} X$ are equivalent to suitable modules for the graded Rees algebra $\mathcal{R}_{X}$. To arrive at this, we first described all $S^{1}$-equivariant sheaves in terms of the extended de Rham algebra $\left.\Omega_{X}^{-} \bullet d\right]$, and then applied Koszul duality with respect to the $\Omega_{X}^{-\bullet}[d]$-augmentation module $\mathcal{O}_{X}$.

For $\Omega S^{2}$-equivariant sheaves, we have a more direct path. Following $[\mathrm{BB}$, we define the complete subprincipal graded Rees algebra to be the base change

$$
\widehat{\mathcal{R}}_{X}^{s p}=\widehat{\mathcal{R}}_{X} \otimes_{\mathcal{O}\left(B S^{1}\right)} \mathcal{O}\left(S^{2}\right) \simeq \widehat{\mathcal{R}}_{X} \otimes_{k[u]} k[u] /\left(u^{2}\right)
$$

over the central parameter. We write $\widehat{\mathcal{R}}_{X}^{s p}$-cmod for the stable $\infty$-category of quasicoherent sheaves on $X$ equipped with a compatible structure of complees $\mathcal{R}_{X}^{s p}$-module. As before, we also 
append the subscript $\mathbf{Z}$ to the notation to denote compatibly $\mathbf{Z}$-graded modules. The following results from Theorem 1.10 by considering the natural $\Omega_{X, S^{2}}^{-\bullet}$-augmentation module $\mathcal{O}_{X} \otimes \mathcal{O}\left(S^{2}\right)$.

Corollary 1.17. For a smooth underived scheme $X$, there are canonical equivalences

$$
\begin{gathered}
\mathrm{QC}(\mathcal{L} X)^{\Omega S^{2}} \simeq \widehat{\mathcal{R}}_{X}^{s p} \text {-mod } \\
\mathrm{QC}(\mathcal{L} X)^{\mathrm{Aff}\left(\Omega S^{2}\right) \rtimes \mathbb{G}_{m}} \simeq \widehat{\mathcal{R}}_{X}^{s p}-\bmod _{\mathbf{z}}
\end{gathered}
$$

Alternatively, we can consider the natural $\Omega_{X, S^{2}}^{-\bullet}$-augmentation module $\Omega_{X}^{-\bullet}$. Let $\Omega_{X}^{-\bullet}\langle d\rangle$ be the $\mathbf{Z}$-graded sheaf of differential graded $k$-algebras on $X$ obtained from the de Rham algebra by adjoining an element $d$ of degree -1 with $[d, \omega]=d \omega$, for $\omega \in \Omega_{X}^{-\bullet}$, but with no equation on powers of $d$. We write $\Omega_{X}^{-\bullet}\langle d\rangle$-cmod for the stable $\infty$-category of quasicoherent sheaves on $X$ equipped with a compatible structure of complete differential graded $\Omega_{X}^{-\bullet}\langle d\rangle$-module. As before, we also append the subscript $\mathbf{Z}$ to the notation to denote compatibly $\mathbf{Z}$-graded modules.

Corollary 1.18. For a smooth underived scheme $X$, there are canonical equivalences

$$
\begin{gathered}
\mathrm{QC}(\mathcal{L} X)^{\mathrm{Aff}\left(\Omega S^{2}\right)} \simeq \Omega_{X}^{-\bullet}\langle d\rangle-\operatorname{cmod} \\
\mathrm{QC}(\mathcal{L} X)^{\mathrm{Aff}\left(\Omega S^{2}\right) \rtimes \mathbb{G}_{m}} \simeq \Omega_{X}^{-\bullet}\langle d\rangle-\operatorname{cmod}_{\mathbf{Z}}
\end{gathered}
$$

Recall the discussed relationship between quasicoherent sheaves $\mathcal{E}$ with flat connection $\nabla$ on $X$ and modules for $\Omega_{X}^{-\bullet}[d]$. It generalizes to a similar relationship between quasicoherent sheaves $\mathcal{E}$ with not necessarily flat connection $\nabla$ and modules for $\Omega_{X}^{-\bullet}\langle d\rangle$. Namely, the connection $\nabla: \mathcal{E} \rightarrow \mathcal{E} \otimes \Omega_{X}$ naturally extends to an $\Omega_{X}^{-\bullet}\langle d\rangle$-module structure on the pullback

$$
\pi^{*} \mathcal{E}=\mathcal{E} \otimes_{\mathcal{O}_{X}} \Omega_{X}^{-\bullet}
$$

The action of the distinguished element $d$ is given by the formula

$$
d(\sigma \otimes \omega)=\nabla(\sigma) \wedge \omega+\sigma \otimes d \omega, \quad \sigma \otimes \omega \in \mathcal{E} \otimes_{\mathcal{O}_{X}} \Omega_{X}^{-\bullet} .
$$

Here we do not require that $\nabla$ is flat nor correspondingly that the square of $d$ vanishes. The square of $d$ is given by the curvature $R=\nabla^{2}: \mathcal{E} \rightarrow \mathcal{E} \otimes \Omega_{X}^{\wedge}$.

1.6.5. Curvature. Before continuing on, we pause here to informally interpret the curvature of connections from the viewpoint of loop spaces. We will place ourselves in the context of the above discussed Koszul duality, and in particular only have in mind sufficiently finite modules.

Let us return to the Hopf fibration $S^{1} \rightarrow S^{3} \rightarrow S^{2}$ and consider its rotation

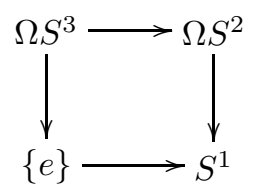

Since the functor $\Omega$ preserves limits, the above is a pullback square of monoids.

On the one hand, by restriction, an $\Omega S^{2}$-equivariant sheaf $\mathcal{F}$ on $\mathcal{L} X$ may be considered as an $\Omega S^{3}$-equivariant sheaf. Note that the $\Omega S^{3}$-action on the loop space is trivial, since the $\Omega S^{2}$-action descends to $S^{1}$. Thus the $\Omega S^{3}$-equivariant structure on $\mathcal{F}$ is in fact $\mathcal{O}_{\mathcal{L X}}$-linear. Moreover, since $\Omega S^{3} \simeq F\left(S^{2}\right)$ is free, the $\Omega S^{3}$-equivariant structure is nothing more than a degree 2 endomorphism of $\mathcal{F}$. When $\mathcal{F}$ is the pullback of a sheaf $\mathcal{E}$ on $X$ with $\Omega S^{2}$-equivariant structure given by a connection $\nabla$ on $\mathcal{E}$, it is immediate that the endomorphism is precisely multiplication by the curvature form of $\nabla$.

On the other hand, the rotated Hopf fibration is not a pushout diagram of monoids. In fact, a resolution of $S^{1}$ by free monoids has infinite length, since $B S^{1}=\mathbf{C P}^{\infty}$ is not a finite 
complex. Given a space with $\Omega S^{2}$-action, or in other words, a space over $B\left(\Omega S^{2}\right)=\mathbf{C P}^{1}$, the induced $\Omega S^{3}$-action is the obstruction to extending to a space over the projective plane $\mathbf{C P}^{2}$. After this, there still remain infinitely many obstructions to overcome to extend to a space over $B S^{1}=\mathbf{C P}^{\infty}$, or in other words, a space with $S^{1}$-action. (These obstructions are related to the Massey products studied in $[\mathrm{St}$.) Nevertheless, in our setting a strong formality statement holds, resulting in the contractibility of all higher data. Namely, in proving Theorem 1.5 we show that an $\Omega S^{2}$-equivariant sheaf on $\mathcal{L} X$ equipped with a trivialization of the equivariant structure of the kernel $\Omega S^{3}$ canonically descends to an $S^{1}$-equivariant sheaf. In down to earth terms, a flat connection is simply a connection with vanishing curvature form.

Now consider a quasicoherent sheaf $\mathcal{E}$ with connection $\nabla$ on $X$. Assume that the curvature $R=\nabla^{2}: \mathcal{E} \rightarrow \mathcal{E} \otimes \Omega_{X}^{\wedge}$ is central (or scalar) in the sense that it factors as a tensor product $R=\operatorname{id}_{\mathcal{E}} \otimes \omega$, for some $\omega \in \Omega_{X}^{\wedge 2}$. Then the $\Omega_{X}^{-\bullet}\langle d\rangle$-module structure on the pullback

$$
\pi^{*} \mathcal{E}=\mathcal{E} \otimes_{\mathcal{O}_{X}} \Omega_{X}^{-\bullet}
$$

descends to the localization where $d^{2}$ acts by multiplication by $\omega$. Thus if rather than trivializing the $\Omega S^{3}$-equivariant structure, we insist that it factors through a specified character, then we obtain sheaves with connection with specified central curvature.

To summarize, sheaves with connection on $X$ naturally give $\Omega S^{2}$-equivariant sheaves on $\mathcal{L} X$ with curvature given by the $\Omega S^{3}$-equivariance. When the curvature vanishes, or in other words, the connection is flat, they descend to $S^{1}$-equivariant sheaves. When the curvature is central, they can be understood as $\Omega S^{2}$-equivariant sheaves on which $\Omega S^{3}$ acts by a character.

1.7. Interpretation: categorified cyclic homology. For the moment, let us suspend the assumption that we are working over a fixed $\mathbf{Q}$-algebra $k$. The definitions and results quoted immediately below hold over any derived ring (and ultimately over the sphere spectrum).

The $\infty$-category $\mathrm{QC}(\mathcal{L} X)$ of quasicoherent sheaves on a loop space $\mathcal{L} X$ carries a rich collection of structures in addition to loop rotation. In a joint paper with J. Francis [BFN], we studied the structures on $\mathrm{QC}(\mathcal{L} X)$ induced by the tensor product on $\mathrm{QC}(X)$ and the composition of loops $\mathcal{L} X \times_{X} \mathcal{L} X \rightarrow \mathcal{L} X$. We introduced the class of perfect stacks which includes all (quasicompact, quasiseparated) schemes, and most commonly arising stacks in characteristic zero. We then established the following characterization of $\mathrm{QC}(\mathcal{L} X)$ for a perfect stack $X$, as an object of the symmetric monoidal $\infty$-category $\mathcal{S} t$ of presentable stable $\infty$-categories.

Theorem 1.19 ([BFN]). Let $X$ be a perfect stack. The $\infty$-category $\mathrm{QC}(X)$ is self-dual as an object of $\mathcal{S}$. The $\infty$-category $\mathrm{QC}(\mathcal{L} X)$ is naturally equivalent both to the Hochschild cohomology category (or Drinfeld center) and the Hochschild homology category of $\mathrm{QC}(X)$ as an algebra in $\mathcal{S}$ t. As a Drinfeld center, $\mathrm{QC}(\mathcal{L} X)$ is endowed with a canonical $\mathcal{E}_{2}$ (or braided tensor) structure, and this structure agrees with the $\mathcal{E}_{2}$-structure induced by the composition of loops.

Thus in the context of the above theorem, we can view Theorem 1.5 as a statement about the categorified version of the cyclic structure on Hochschild chains discovered by Connes [Co].

To explain this, let $\mathcal{C}$ denote a symmetric monoidal $(\infty, 1)$-category and $A \in \mathcal{C}$ an algebra object. The Hochschild homology $H H(A)$ of $A$ is derived abelianization of $A$, i.e., the pairing of $A$ with itself, considered as left and right module over its enveloping algebra $A \otimes A^{o p}$ :

$$
H H(A)=A \otimes A \otimes A^{o p} A,
$$

or explicitly as the geometric realization of the cyclic bar construction of $A$. For $A$ commutative this has the alternative description $H H(A)=S^{1} \otimes A$ in terms of the tensoring of commutative algebras over simplicial sets, in particular revealing an $S^{1}$ action on Hochschild homology. This $S^{1}$ action can be extended to arbitrary associative $A$ through the cyclic symmetry of the cyclic 
bar construction, or geometrically thanks to the theory of topological chiral homology (an aspect of topological field theory), which realizes $H H(A)$ as an integration $H H(A)=\int_{S^{1}} A$ of $A$ over $S^{1}$, see [L2, Theorem 5.3.3.11].

Definition 1.20. The cyclic homology of $A$ is the $S^{1}$-invariants $H H(A)^{S^{1}}$.

Now let us specialize the above to the context of this paper, namely where we work over a fixed $\mathbf{Q}$-algebra $k$. Then the results of this paper and [BFN] combine to describe the cyclic homology of $\mathrm{QC}(X)$ thought of as an algebra object in the $\infty$-category $\mathcal{S} t_{k}$ of presentable stable $k$-linear $\infty$-categories.

Corollary 1.21. For $X$ a quasicompact quasiseparated derived scheme over $k$, the $k$-linear cyclic homology of $\mathrm{QC}(X)$ is canonically equivalent to the $\infty$-category $\Omega_{X}^{-\bullet}[d]-\bmod$ of de Rham modules on $X$.

Our results in Section 1.6 .3 show that $\Omega_{X, d^{-}}^{-\boldsymbol{e}}$-modules, or $\mathcal{D}_{X}$-modules, are an analogue of periodic cyclic homology, and moreover, endow it with a Hodge filtration given by the $\infty$ category of $B \mathbb{G}_{a} \rtimes \mathbb{G}_{m}$ equivariant sheaves on the loop space, or Rees modules.

We can also view the cyclic homology of $\mathrm{QC}(X)$ as an invariant of its $\infty$-category of module categories, which are "derived categorical sheaves" over $X$. In particular, it plays a central role as the natural recipient for the Chern character from the secondary K-theory of $X$ in the theory developed by Toën and Vezzosi, see ToVe3, ToVe4].

1.8. Loops in derived stacks. We now consider the generalizations of our results from schemes to stacks. We will only consider geometric stacks $X$, by which we connote an Artin derived stack with affine diagonal. (We refer the reader to the survey To2 for an overview of Artin stacks and [HAG2] for detailed foundations, in particular the theory of tangent complexes.) Concretely, such stacks have an affine cover $U$ which is smooth and affine over $X$. Thus $X$ can be written as the geometric realization $X \simeq\left|U_{\bullet}\right|$ of the associated Cech cover, the simplicial affine scheme with $k$-simplices given by $k+1$-fold fiber products $U \times_{X} \cdots \times_{X} U$ and with smooth structure maps. (One could in fact use the existence of such presentations as the definition of a geometric stack.) This allows us to reduce questions on $X$ to questions on affine schemes which are local in the smooth topology.

The fundamental difference between loops in schemes and stacks is a consequence of the well known fact that loops in a topological space $X$ are non-local objects: a loop in $X$ does not always come from a loop in a cover of $X$, since a "big" loop can not be expressed in terms of "small" loops. Similarly, loops in a derived stack $X$ are not local objects with respect to the étale topology (not to mention smooth or flat topologies). In other words, one does not expect to be able to recover the loop space $\mathcal{L} X$ from the loop space $\mathcal{L} U$ of a cover $U \rightarrow X$, and the assignment of loops $U \mapsto \mathcal{L} U$, for étale maps $U \rightarrow X$, does not in general form a cosheaf. This makes the theory of loops in stacks much more intricate than that of schemes.

If we restrict to "small" loops, then it is not unreasonable to think that we can recover small loops in $X$ from small loops in a cover. While this statement is not true on the level of points, we will show that locality holds for small loops on the level of functions and sheaves.

Let's illustrate the basic phenomena with the example $X=B G=p t / G$ of the classifying stack of an algebraic group $G$. In this case, it is easy to see that the loop space is the adjoint quotient

$$
\mathcal{L} B G=G / G
$$

This is the stack of $G$-local systems on the circle (for example, rank $n$ local systems for $G=$ $\left.G L_{n}\right)$. As an illustration of the non-local behavior of loops, note that loops $\mathcal{L}(p t)=p t$ in the cover $p t \rightarrow B G$ can not see nontrivial conjugacy classes in $\mathcal{L} X \simeq G / G$. 
Similarly, for a general geometric stack $X$, with underlying underived stack $\underline{X}$, the loop space $\mathcal{L} X$ is a derived thickening of the inertia stack $I \underline{X}$. The inertia stack is an affine group scheme over $\underline{X}$, parametrizing points of $\underline{X}$ together with automorphisms.

Unlike for schemes, the loop space of a geometric stack is also quite different from its odd tangent bundle. For example, in the case $X=B G$, the latter is the adjoint quotient of the Lie algebra

$$
\mathbb{T}_{B G}[-1]=\mathfrak{g} / G
$$

To interpolate between loops and odd tangents on geometric stacks, we introduce the space of formal loops.

Definition 1.22. The formal loop space $\widehat{\mathcal{L}} X$ of a derived stack $X$ is the formal completion $\widehat{\mathcal{L X}}_{X}$ of the loop space along the constant loops $X \rightarrow \mathcal{L} X$.

To continue the above example, the formal completion of $\mathcal{L} B G$ along the constant loops is the adjoint quotient of the formal group

$$
\widehat{\mathcal{L}} B G \simeq \widehat{G} / G .
$$

Furthermore, the exponential map provides a canonical identification

$$
\widehat{\mathfrak{g}} / G \stackrel{\sim}{\longrightarrow} \widehat{G} / G
$$

with the formal completion of the shifted tangent bundle along its zero section.

We show in Theorem 6.9 that this picture extends to arbitrary geometric stacks.

Theorem 1.23. There is a canonical equivalence

$$
\exp : \widehat{\mathbb{T}}_{X}[-1] \longrightarrow \widehat{\mathcal{L}} X
$$

from the formal odd tangent bundle to the formal loop space of $X$.

There is also an intermediate notion of loop space suggested by rational homotopy theory. The algebra of $k$-linear cochains $C^{*}(M, k)$ on a topological space (which is the algebra of functions $\mathcal{O}(M)$ on the locally constant stack $M$, and hence on its affinization $\operatorname{Aff}(M)$ ) recovers the $k$-homotopy type of $M$ for simply connected $M$. For general $M$, this algebra only sees the pro-unipotent completion of the fundamental group. Namely, $C^{*}(M, k)=\mathcal{O}(M)$ can be described as the self-Exts of the constant sheaf (the trivial local system) on $M$. Thus its modules describe pro-unipotent local systems on $M$. With this picture in mind for $M=S^{1}$, we make the following definition.

Definition 1.24. The unipotent loop space $\mathcal{L}^{u} X=\operatorname{Map}\left(B \mathbb{G}_{a}, X\right)$ is the space of loops which factor through the affinization $\operatorname{Aff}\left(S^{1}\right) \simeq B \mathbb{G}_{a}$ of the circle.

The $S^{1}$-action on the unipotent loop space factors through a $B \mathbb{G}_{a}$-action. Moreover, we have a $\mathbb{G}_{m}$-action coming from rotation of $B \mathbb{G}_{a}$, and the two combine to an action of $B \mathbb{G}_{a} \rtimes \mathbb{G}_{m}$.

In the example $X=B G$, the unipotent loop space classifies group homomorphisms $\mathbb{G}_{a} \rightarrow G$, or in other words, algebraic one-parameter subgroups, up to conjugation. (For $G=G L_{n}$ these are unipotent rank $n$ local systems on the circle.) Thus we find

$$
\mathcal{L}^{u} B G \simeq G^{u} / G
$$

where $G^{u}$ is the formal neighborhood of the unipotent cone of $G$ (so the reduced points of $G^{u}$ are unipotent elements of $G$ ). Since any Lie algebra element formally exponentiates to a one-parameter subgroup, we have a canonical inclusion

$$
\widehat{\mathcal{L}} B G \subset \mathcal{L}^{u} B G .
$$


(In particular, note that the unipotent loop space for $B G$ is not reduced!) We find in Theorem 6.9 that this picture too extends to all geometric stacks:

Theorem 1.25. Formal loops are unipotent: the canonical inclusion $\widehat{\mathcal{L}} X \rightarrow \mathcal{L} X$ factors through the canonical map $\mathcal{L}^{u} X \rightarrow \mathcal{L} X$ into a sequence

$$
\widehat{\mathcal{L}} X \longrightarrow \mathcal{L}^{u} X \longrightarrow \mathcal{L} X .
$$

In particular, $\widehat{\mathcal{L}} X$ inherits a $B \mathbb{G}_{a} \rtimes \mathbb{G}_{m}$-action from $\mathcal{L}^{u} X$.

1.8.1. Descent for formal loops. None of our three notions of loop space is truly local. Nevertheless, in Section 6.2 we find that the algebra of functions on formal loops is a local object. Namely, we first show that Hochschild chains (or equivalently, differential forms) on a derived ring satisfy smooth descent. (The assertion of étale descent for the Hochschild chain complex is a theorem of Weibel and Geller WG.)

Proposition 1.26. The assignment of Hochschild chains

$$
R \longmapsto H C(R)=R \otimes S^{1}=R \otimes_{R \otimes R} R \simeq \operatorname{Sym}^{\bullet}\left(\Omega_{R}[1]\right)
$$

satisfies smooth descent: it forms a sheaf with respect to the smooth topology on derived rings.

To extend this to a sheaf on geometric stacks, we need to observe that for a derived ring $R$, Hochschild chains $H C(R) \simeq \operatorname{Sym}^{\bullet}\left(\Omega_{R}[1]\right)$ are automatically complete. Equivalently, the loop space $\mathcal{L} \operatorname{Spec} R$ of an affine derived scheme is a formal thickening of $\operatorname{Spec} R$. As can be seen from the example $B G$, this is no longer true for general stacks. Instead, the sheaf extending Hochschild chains on affines is given by the completed symmetric algebra of one-forms. We will refer to the formal completion of the odd tangent bundle along the zero section as the formal odd tangent bundle

$$
\widehat{\mathbb{T}}_{X}[-1]=\operatorname{colim}_{n \rightarrow \infty} \operatorname{Spec}_{X} \operatorname{Sym}^{\bullet}\left(\Omega_{X}[1]\right) / \operatorname{Sym}^{>n}\left(\Omega_{X}[1]\right) .
$$

We establish the following descent results for completed algebras of forms.

Theorem 1.27. Fix a geometric stack $X$.

(1) The assignment of the complete graded algebra of differential forms

$$
U \longmapsto \lim _{n \rightarrow \infty} \operatorname{Sym}^{\bullet}\left(\Omega_{U}[1]\right) / \operatorname{Sym}^{>n}\left(\Omega_{U}[1]\right)
$$

defines a sheaf on the smooth site of $X$.

(2) The assignment of complete compatibly graded modules

$$
U \longmapsto \mathrm{QC}\left(\widehat{\mathbb{T}}_{U}[-1]\right)^{\mathbb{G}_{m}}
$$

forms a sheaf of $\infty$-categories with $B \mathbb{G}_{a}$-action on the smooth site of $X$.

Theorems 1.23 and 1.27 together establish that the formal loop space behaves like a local construction from the point of view of functions and sheaves. For a smooth cover $U \rightarrow X$, with corresponding Cech simplicial scheme $U_{\bullet}$, we have the local-to-global identities

$$
\mathcal{O}_{\widehat{\mathcal{L}} X} \simeq \lim \mathcal{O}\left(\widehat{\mathcal{L}} U_{\bullet}\right) \quad \mathrm{QC}(\widehat{\mathcal{L}} X)^{\mathbb{G}_{m}} \simeq \lim \mathrm{QC}\left(\widehat{\mathcal{L}} U_{\bullet}\right)^{\mathbb{G}_{m}}
$$

compatible in the first case with the de Rham vector field, and in the second case with the resulting $B \mathbb{G}_{a}$-action.

With the latter identity in hand, our results on loop spaces of derived schemes, in particular Theorems 1.5 and 1.10 and their corollaries, extend to formal loop spaces of smooth underived 
geometric stacks. For example, we have the identification of $S^{1}$-equivariant sheaves on formal loop spaces

$$
\begin{gathered}
\mathrm{QC}(\widehat{\mathcal{L}} X)^{S^{1}} \simeq \Omega_{X}^{-\bullet}[d]-\bmod \\
\mathrm{QC}(\widehat{\mathcal{L}} X)^{B \mathbb{G}_{a} \rtimes \mathbb{G}_{m}} \simeq \Omega_{X}^{-\bullet}[d]-\bmod _{\mathbf{z}}
\end{gathered}
$$

and the identification of $\Omega S^{2}$-equivariant sheaves on formal loop spaces

$$
\begin{gathered}
\mathrm{QC}(\widehat{\mathcal{L}} X)^{\Omega S^{2}} \simeq \Omega_{X}^{-\bullet}\langle d\rangle-\operatorname{cmod} \\
\mathrm{QC}(\widehat{\mathcal{L}} X)^{\mathrm{Aff}\left(\Omega S^{2}\right) \rtimes \mathbb{G}_{m}} \simeq \Omega_{X}^{-\bullet}\langle d\rangle-\operatorname{cmod}_{\mathbf{Z}}
\end{gathered}
$$

Furthermore, we have the Koszul dual formulation for $S^{1}$-equivariant sheaves

$$
\begin{gathered}
\mathrm{QC}(\widehat{\mathcal{L}} X)^{B \mathbb{G}_{a}} \simeq \widehat{\mathcal{R}}_{X-\operatorname{cmod}} \\
\mathrm{QC}(\widehat{\mathcal{L}} X)^{B \mathbb{G}_{a} \rtimes \mathbb{G}_{m}} \simeq \widehat{\mathcal{R}}_{X^{-}-\operatorname{cmod}_{\mathbf{Z}}}
\end{gathered}
$$

and similarly for $\Omega S^{2}$-equivariant sheaves

$$
\begin{gathered}
\mathrm{QC}(\widehat{\mathcal{L}} X)^{\Omega S^{2}} \simeq \widehat{\mathcal{R}}_{X}^{s p} \text {-cmod } \\
\mathrm{QC}(\widehat{\mathcal{L}} X)^{\mathrm{Aff}\left(\Omega S^{2}\right) \rtimes \mathbb{G}_{m}} \simeq \widehat{\mathcal{R}}_{X}^{s p}-\operatorname{cmod}_{\mathbf{z}}
\end{gathered}
$$

Finally, to state an analogue of Corollary 1.14 for perfect modules, for simplicity let us restrict to a situation tailored to many applications. Suppose that $\mathcal{L} X$ turns out to be underived in the sense that its structure sheaf is discrete. Let us write $\operatorname{Perf}_{X}(\widehat{\mathcal{L}} X)$ for the small, stable $\infty$-category of quasicoherent sheaves whose restriction along the canonical map $X \rightarrow \widehat{\mathcal{L}} X$ are perfect. (We caution the reader that this is not the same as the previously introduced small, stable $\infty$-category $\operatorname{Perf}_{X}(\mathcal{L} X)$ of quasicoherent sheaves whose pushforward along the canonical map $\mathcal{L} X \rightarrow X$ are perfect.) As usual, we write $\mathcal{R}_{X}$-perf for the stable $\infty$-category of quasicoherent sheaves on $X$ equipped with a compatible structure of perfect $\mathcal{R}_{X}$-module. Under the assumption that $\mathcal{L} X$ is underived, we have the further identifications

$$
\begin{gathered}
\operatorname{Perf}_{X}(\widehat{\mathcal{L}} X)^{B \mathbb{G}_{a} \rtimes \mathbb{G}_{m}} \simeq \mathcal{R}_{X} \text {-perf }_{\mathbf{Z}} \\
\operatorname{Perf}_{X}(\widehat{\mathcal{L}} X)^{B \mathbb{G}_{a}} \simeq \mathcal{R}_{X} \text {-perf }
\end{gathered}
$$

1.8.2. Sheaves on unipotent loops. Finally, there is a useful version of the above story for unipotent loops (in place of formal loops). Let us continue with the assumption that $\mathcal{L} X$ is underived in the sense that its structure sheaf is discrete.

Let $\operatorname{Perf}_{X}\left(\mathcal{L}^{u} X\right)$ denote the subquotient $\infty$-category of $\mathrm{QC}\left(\mathcal{L}^{u} X\right)$ where we kill the kernel of the restriction along the canonical map $X \rightarrow \mathcal{L}^{u} X$, and require that the restriction produces perfect sheaves on $X$. Then there is a canonical equivalence

$$
\operatorname{Perf}_{X}(\widehat{\mathcal{L}} X) \simeq \operatorname{Perf}_{X}\left(\mathcal{L}^{u} X\right)
$$

Note that objects on the two sides admit the same characterization since there is no kernel to restriction along the canonical map $X \rightarrow \widehat{\mathcal{L}} X$.

Putting this together with our previous results provides the following.

Theorem 1.28. For a smooth geometric stack $X$ such that $\mathcal{L} X$ is underived, there are canonical equivalences

$$
\begin{gathered}
\operatorname{Perf}_{X}\left(\mathcal{L}^{u} X\right)^{B \mathbb{G}_{a} \rtimes \mathbb{G}_{m}} \simeq \mathcal{R}_{X}-\operatorname{perf}_{\mathbf{Z}} \\
\operatorname{Perf}_{X}\left(\mathcal{L}^{u} X\right)^{B \mathbb{G}_{a}} \simeq \mathcal{R}_{X} \text {-perf }
\end{gathered}
$$


As a consequence, our results on sheaves on formal loop spaces generalize to sheaves on unipotent loop spaces once we insist on $\mathbb{G}_{m}$-equivariance and bounded weights. This result will be applied in [BN2] to situations of interest in representation theory (such as equivariant Steinberg varieties).

Acknowledgments We have benefited tremendously from many conversations on the subjects of loop spaces, cyclic homology and homotopy theory. We would like to thank Kevin Costello, Ian Grojnowski, David Kazhdan, Mike Mandell, Tom Nevins, Tony Pantev, Brooke Shipley, Dima Tamarkin, Constantin Teleman, Boris Tsygan, Amnon Yekutieli, and Eric Zaslow for their many useful comments. We would like to especially thank Andrew Blumberg for his explanations of homotopical algebra, and Jacob Lurie and Bertrand Toën for introducing us to the beautiful world of derived algebraic geometry and patiently answering our many questions. Finally, we are very grateful to a conscientious referee who provided detailed comments on a first submitted version.

The first author was partially supported by NSF grant DMS-0449830, and the second author by NSF grant DMS-0600909. Both authors would also like to acknowledge the support of DARPA grant HR0011-04-1-0031.

\section{Preliminaries}

2.1. $\infty$-categories. Roughly speaking, an $\infty$-category (or synonymously $(\infty, 1)$-category) encodes the notion of a category whose morphisms form topological spaces and whose compositions and associativity properties are defined up to coherent homotopies. They offer a context for homotopy theory which lies between the coarse world of homotopy categories and the fine world of model categories. Ordinary categories fit into this formalism as discrete $\infty$-categories.

The theory of $\infty$-categories has many alternative formulations (as topological categories, Segal categories, quasicategories, etc; see $\overline{B e r}$ for a comparison between the different versions). We will follow the conventions of [L1], which is based on Joyal's quasi-categories [Jo. Namely, an $\infty$-category is a simplicial set satisfying a weak version of the Kan condition guaranteeing the fillability of inner horns. The underlying vertices play the role of the set of objects while the fillable inner horns correspond to sequences of composable morphisms. The book [L1] presents a detailed study of $\infty$-categories, developing analogues of many of the common notions of category theory. An overview of the $\infty$-categorical language, including limits and colimits, appears in L1, Chapter 1.2]. $\infty$-categories and the more traditional settings of model categories or homotopy categories is that coherent homotopies are naturally built into the definitions. Thus for example, all functors are derived and the natural notions of limits and colimits correspond to homotopy limits and colimits.

To pursue homotopical algebra within this formalism, one needs a theory of monoidal and symmetric monoidal $\infty$-categories. Such a theory is developed in [L2, 2,4] including appropriate notions of algebra objects, module categories over algebra objects, and so on. The setting of stable $\infty$-categories, as developed in [L2, 1], provides an analogue of the additive setting of homological algebra. A stable $\infty$-category can be defined as an $\infty$-category with a zeroobject, closed under finite limits and colimits, and in which pushouts and pullbacks coincide [L2, 1.1.3]. A key result of [L2, 1.1.2] is that stable $\infty$-categories are enhanced versions of triangulated categories, in the sense that their homotopy categories are canonically triangulated. A representative example is the $\infty$-categorical enhancement of the derived category of modules over a ring.

Given a triangulated category which is linear over a commutative $\mathbf{Q}$-algebra $k$, we may consider enhancing its structure in three equivalent ways, promoting it to (1) a differential graded (dg) category, (2) an $A_{\infty}$-category, or (3) an $\infty$-category. (Among the many excellent 
references for dg and $A_{\infty}$-categories, we recommend the survey [Ke.) In particular, $k$-linear stable $\infty$-categories are equivalent to $k$-linear pre-triangulated $\mathrm{dg}$ categories (that is, those whose homotopy category is triangulated) and the reader may substitute the latter for the former throughout the present paper. (See [BGT, Theorem 3.19] for a general equivalence between stable linear categories and spectral categories which reduces to the above assertion for the Eilenberg-Mac Lane spectrum of a $\mathbf{Q}$-algebra $k$.)

The collection $\mathcal{S} t_{k}$ of stable presentable $k$-linear $\infty$-categories itself forms a symmetric monoidal $\infty$-category. To see this, recall that the $\infty$-category of stable presentable $\infty$-categories is symmetric monoidal [L2, 6.3.2]. It contains the $\mathcal{E}_{\infty}$-algebra object $\operatorname{Mod}_{k}$ of modules over the commutative ring $k$. By definition, the $\infty$-category $\mathcal{S} t_{k}$ is the module category $\operatorname{Mod}_{M^{\prime} d_{k}}(\mathcal{S} t)$ of $\operatorname{Mod}_{k}$-modules inside of $\mathcal{S} t$. Thus $\mathcal{S t}_{k}$ inherits a symmetric monoidal structure given by tensoring over the $\mathcal{E}_{\infty}$-algebra object $\operatorname{Mod}_{k}$.

Note as well that the forgetful functor $\mathcal{S} t_{k} \rightarrow \mathcal{S} t$ is conservative: a $k$-linear functor $F$ : $\mathcal{C} \rightarrow \mathcal{D}$ is an equivalence if and only if it is an equivalence as a functor of stable $\infty$-categories. Furthermore, in the $\infty$-setting, stability is a property not a structure, so it suffices in turn to ask if $F$ is an equivalence on plain $\infty$-categories.

2.2. Derived stacks. Derived algebraic geometry provides a useful language to discuss various objects and constructions of representation theory and topological field theory. The basic objects are derived stacks which are built out of affine derived schemes in the same way that higher stacks are built out of ordinary affine schemes. In this paper, we are primarily interested in stable $\infty$-categories of quasicoherent sheaves on derived stacks. We will discuss below what we mean by affine derived scheme, and then briefly review the notion of derived stack and quasicoherent sheaf.

For more details on derived algebraic geometry, we refer the reader to Toën's extremely useful survey [To2, and to the papers of Toën-Vezzosi [HAG1, HAG2] and Lurie [L1, L2, L3]. In particular, we were introduced to derived loop spaces by [To2].

2.2.1. Derived rings. Derived algebraic geometry is a form of algebraic geometry in which the usual notion of discrete commutative ring is replaced by a homotopical generalization. There is not a single choice of generalization, but a variety of notions suited to different applications. All the possible versions of commutative rings we might consider can be interpreted as commutative ring objects (satisfying some further conditions) in some symmetric monoidal $\infty$-category $\mathcal{C}$.

For our intended applications in representation theory and topological field theory, the most relevant choice is to fix a discrete commutative algebra $k$ over the rational numbers, and take $\mathcal{C}$ to be the stable symmetric monoidal $\infty$-category $\operatorname{Mod}_{k}$ of cochain complexes of $k$-modules (localized with respect to quasi-isomorphisms). Thanks to [L2, Theorem 4.4.4.7], one can regard a commutative ring object in $\operatorname{Mod}_{k}$ as nothing more than a commutative dg $k$-algebra. We will further insist that all such rings be connective in the sense that they are cohomologically trivial in positive degrees.

Definition 2.1. By a derived $k$-algebra, we mean a connective commutative dg $k$-algebra, where $k$ is a rational commutative algebra. We write $D G A^{-}$for the $\infty$-category of derived $k$-algebras.

We will also write $D G A_{k}$ for the corresponding $\infty$-category of arbitrary (not necessarily connective) commutative dg $k$-algebras.

Remark 2.2. If $k$ is a discrete commutative algebra over the rational numbers, then the stable symmetric monoidal $\infty$-categories of cochain complexes of $k$-modules, simplicial $k$-modules, and 
$k$-modules in spectra are all equivalent. This devolves from an underlying Quillen equivalence of model categories. Thus there is a single common notion of derived $k$-algebra.

2.2.2. Derived stacks. Fix once and for all a discrete commutative rational algebra $k$.

We begin with a reminder of the functor of points for higher stacks. Let $\mathcal{S}$ denote the $\infty$ category of simplicial sets, or equivalently (compactly generated Hausdorff) topological spaces. Let $A l g_{k}$ denote the category of discrete commutative $k$-algebras. By definition, the category of affine schemes over $k$ is the opposite category $A l g_{k}^{o p}$. A stack over $k$ is a functor

$$
X: \operatorname{Alg}_{k} \rightarrow \mathcal{S}
$$

which is a sheaf in the étale topology. This means that $X$ preserves those colimits corresponding to étale covers of affine schemes. We write $S t a c k_{k}$ for the $\infty$-category of stacks over $k$.

Familiar examples of stacks include ordinary schemes and Deligne-Mumford or Artin stacks in groupoids. At the other extreme, topological spaces $K \in \mathcal{S}$ naturally define stacks, as the sheafification of constant functors $K: A l g_{k} \rightarrow \mathcal{S}$. One can choose a simplicial presentation of $K$ (for example, that given by singular chains) and consider $K$ as a functor to simplicial sets. Of course, any two simplicial presentations lead to equivalent stacks. The inclusion of schemes into stacks valued in discrete sets preserves limits, such as fiber products, but alters colimits, such as group quotients.

Definition 2.3. The $\infty$-category of affine derived schemes over $k$ is the opposite $\infty$-category $\left(D G A_{k}^{-}\right)^{o p}$ to derived $k$-algebras.

The Yoneda embedding gives a fully faithful embedding of $\left(D G A_{k}^{-}\right)^{o p}$ into the $\infty$-category of functors $D G A_{k}^{-} \rightarrow \mathcal{S}$. We will be interested in gluing together affine derived schemes into more general functors.

Definition 2.4. A derived stack over $k$ is a functor

$$
X: D G A_{k}^{-} \rightarrow \mathcal{S}
$$

which is a sheaf in the étale topology, so in other words, $X$ preserves those colimits corresponding to étale covers of affine derived schemes. We write $D S t_{k}$ for the $\infty$-category of derived stacks over $k$.

Note that $A l g_{k}$ sits as a full subcategory of $D G A_{k}^{-}$, and its inclusion preserves limits (though not colimits). Equivalently, ordinary affine schemes sit as a full subcategory of affine derived schemes, and their inclusion preserves colimits (though not limits). This enables us to relate derived and underived stacks by adjoint functors:

$$
i: S t a c k_{k} \longleftrightarrow D S t_{k}: r
$$

where $i$ is fully faithful. The functor $r$ takes a derived stack and restricts its domain from $D G A_{k}^{-}$to $A l g_{k}$. It admits a left adjoint $i$ which extends the embedding of affine schemes into derived stacks by continuity (that is, to preserve colimits). In other words, given a stack $X$, we write $X$ as a colimit of ordinary affine schemes Spec $R_{\alpha}$, and then $i(X)$ is the colimit of the same affine schemes Spec $R_{\alpha}$ but now considered as derived stacks. Thus considering stacks as derived stacks preserves the notion of colimit but typically alters the notion of limit. For instance, the fiber product of affine schemes

$$
\operatorname{Spec} R_{1} \times_{\operatorname{Spec}} R_{0} \operatorname{Spec} R_{2} \in D S t_{k}
$$

considered as derived stacks is given by the spectrum of the derived tensor (or Tor) algebra

$$
R_{1} \otimes_{R_{0}} R_{2} \in D G A_{k}^{-} \text {. }
$$


Another important difference between stacks and derived stacks is apparent in the calculation of mapping spaces. The $\infty$-category of derived stacks is naturally a closed symmetric monoidal $\infty$-category, with monoidal structure given by the point-wise Cartesian product of functors. The inner Homs (closed monoidal structure) are given by the sheaf Homs, which are the derived mapping stacks $\operatorname{Map}(X, Y)$. Note that colimits (in particular gluings) in the first factor are converted to limits (in particular fiber products) in the mapping space. Thus when $X=K$ is a simplicial set, built concretely out of discrete sets with identifications, we can think of $\operatorname{Map}(K, Y)$ as a collection of equations imposed on products of copies of $Y$. Even for $Y$ affine this construction may lead to interesting derived structure.

Finally, we will often restrict to the following reasonable class of derived stacks.

Definition 2.5. We use the term geometric stack to refer to an Artin derived stack with affine diagonal.

We refer the reader to the survey [To2 for an overview of Artin stacks. The definition of Artin derived stack is inductive. A -1-Artin derived stack is an affine derived scheme; a morphism of derived stacks is -1-Artin if its fibers over affines are -1-Artin derived stacks. In general, a derived stack $X$ is $n$-Artin if there exists a disjoint union of affine derived schemes $U$ and a smooth $(n-1)$-representable and surjective morphism $U \rightarrow X$; a morphism of derived stacks is called $n$-representable if its fibers over affines are $n$-Artin derived stacks. An Artin derived stack is an $n$-Artin derived stack for some $n$.

2.2.3. Quasicoherent sheaves. To any derived stack $Z \in D S t_{k}$, there is assigned a stable presentable $k$-linear $\infty$-category $\mathrm{QC}(Z)$ called the $\infty$-category of quasicoherent sheaves on $Z$ (see [To2, p. 36], and [L4 for a detailed study). Let us briefly recall its construction.

First, for a derived $k$-algebra $A$, we define $\mathrm{QC}(\operatorname{Spec} A)$ to be the stable $\infty$-category of $A$ modules $\operatorname{Mod}_{A}$. Equivalently, we can take $\operatorname{Mod}_{A}$ to be the dg category of $\operatorname{dg} A$-modules localized with respect to quasi-isomorphisms. Given a map $p: \operatorname{Spec} A \rightarrow \operatorname{Spec} B$, we have the usual pullback functor

$$
p^{*}: \mathrm{QC}(\operatorname{Spec} B) \rightarrow \mathrm{QC}(\operatorname{Spec} A) \quad p^{*}(M)=M \otimes_{B} A
$$

Now in general, for tautological reasons, any derived stack $Z$ is the colimit of the diagram $Z$. of all affine derived schemes over $Z$. We define $\mathrm{QC}(Z)$ to be the limit (in the $\infty$-category $\mathcal{S} t_{k}$ of stable presentable $k$-linear $\infty$-categories) of the corresponding diagram $\mathrm{QC}(Z \bullet)$ with respect to pullback. Thus we can interpret the functor

$$
\mathrm{QC}: D S t_{k} \rightarrow \mathcal{S} t_{k}^{o p}
$$

as the unique colimit-preserving extension of the functor

$$
\text { Mod : }\left(D G A_{k}^{-}\right)^{o p} \rightarrow \mathcal{S} t_{k}^{o p}
$$

that assigns modules to the spectrum of a derived $k$-algebra. In other words, we can view quasicoherent sheaves as the continuous linearization of derived stacks.

2.3. Graded modules. This section contains some technical material needed later in the paper. The reader should probably skip it until needed. We record here some useful structural properties of modules over $\mathbf{Z}$-graded $k$-algebras.

Given a (not necessarily commutative) dg $k$-algebra $R$, we write $R$-mod for the stable $\infty$ category of $\operatorname{dg} R$-modules. Given a $\mathbf{Z}$-graded $\operatorname{dg} k$-algebra $R$, we write $R$ - $\bmod \mathbf{z}$ for the stable $\infty$-category of compatibly $\mathbf{Z}$-graded dg $R$-modules. By descent, working with $\mathbf{Z}$-graded algebras and modules is equivalent to working over the classifying stack $B \mathbb{G}_{m}$. 
We will always use the term degree to refer to cohomological degree, and the term weight to refer to the $\mathbf{Z}$-grading on a $\mathbf{Z}$-graded dg algebra and its $\mathbf{Z}$-graded dg modules. For a $\mathbf{Z}$-graded dg module $M$, we will denote by $M[n]$ the customary degree shift by $-n$, and by $M\langle m\rangle$ the weight shift by $m$. So for example, if $M$ is concentrated in degree 0 and weight 0 , then $M[n]\langle m\rangle$ is concentrated in degree $-n$ and weight $m$.

Suppose $R$ is a $\mathbf{Z}$-graded dg $k$-algebra concentrated in nonnegative weights. We will write $\widehat{R}$ for the completion of $R$ with respect to the positive weight direction. To be precise, for $k>0$, let $R_{>k} \subset R$ denote the ideal of positive weight spaces greater than $k$. We have a natural diagram of dg algebras

$$
R / R_{>0} \longleftarrow R / R_{>1} \longleftarrow R / R_{>2} \longleftarrow \cdots
$$

We take $\widehat{R}$ to be the limit dg algebra of this diagram. We will write $\widehat{R}-\operatorname{cmod}_{\mathbf{z}}$ for the stable $\infty$-category of compatibly $\mathbf{Z}$-graded complete dg $\widehat{R}$-modules. To be precise, the above diagram of $d g$ algebras provides a diagram of stable $\infty$-categories of graded modules

$$
R / R_{>0}-\bmod _{\mathbf{z}} \longrightarrow R / R_{>1}-\bmod _{\mathbf{z}} \longrightarrow R / R_{>2}-\bmod _{\mathbf{Z}} \longrightarrow \cdots
$$

We take $\widehat{R}$-cmodz to be the limit stable $\infty$-category of this diagram.

Finally, given a $\mathbf{Z}$-graded stable $\infty$-category $\mathcal{C}$, we will write $\mathcal{C}_{+} \subset \mathcal{C}$, respectively $\mathcal{C}_{-} \subset \mathcal{C}$, for the full $\infty$-subcategory of objects with weights bounded below, respectively bounded above.

2.3.1. Shear shift. It is useful to recognize that $R-\bmod _{\mathbf{z}}$ is independent of certain shifts. For $i \in \mathbf{Z}$, let $R^{i} \subset R$ denote the summand of weight $i$. For $n \in \mathbf{Z}$, we will write $R_{[n]}$ for the $\operatorname{dg}$ $k$-algebra defined by the shear shifting

$$
R_{[n]}=\oplus_{i \in \mathbf{Z}} R^{i}[n i] .
$$

Of course when $n=0$, we recover our original algebra $R_{[0]}=R$. It is worth commenting that in general if $R$ is commutative, then only the even shifted versions $R_{[2 n]}$ are commutative as well. For example, if $R=k[t]$ with $t$ of degree 0 and weight 1 , then $R_{[2 n]}$ is free commutative, but $R_{[2 n+1]}$ is not commutative.

Now we have the following useful independence of shifts.

Proposition 2.6. For $n \in \mathbf{Z}$, there is a canonical weight-preserving equivalence of stable $\infty$ categories

$$
R-\bmod _{\mathbf{Z}} \simeq R_{[n]}-\bmod _{\mathbf{z}} .
$$

Proof. We will send the free module $R$ to the free module $R_{[n]}$, and then extend preserving colimits and shifts by weight and cohomological degree. To have this make sense, we need only check that the endomorphisms of $R$ and $R_{[n]}$ agree. Considered as plain algebras, $R$ and $R_{[n]}$ of course have endomorphisms $R$ and $R_{[n]}$ themselves respectively. Those endomorphism that survive in the $\mathbf{Z}$-graded categories are the invariants of weight 0 . In both cases, the invariants are simply the weight 0 summand $R^{0}$.

2.3.2. Periodic localization. Consider the $\mathbf{Z}$-graded polynomial algebra $k[t]$ with $t$ of degree 0 and weight 1 . In geometric language, we have $\mathbb{A}^{1}=\operatorname{Spec} k[t]$ equipped with the standard $\mathbb{G}_{m}$-action. Consider as well the $\mathbf{Z}$-graded commutative $k[t]$-algebra $k\left[t, t^{-1}\right]$. In geometric language, we have $\mathbb{G}_{m}=\operatorname{Spec} k\left[t, t^{-1}\right]$ equipped with the standard $\mathbb{G}_{m}$-action. The algebra map $k[t] \rightarrow k\left[t, t^{-1}\right]$ corresponds to the standard $\mathbb{G}_{m}$-equivariant open embedding $\mathbb{G}_{m} \rightarrow \mathbb{A}^{1}$.

Now let $R$ be an arbitrary algebra object in $k[t]-\bmod _{\mathbf{z}}$, so that $R-\bmod _{\mathbf{z}}$ consists of $R$ module objects in $k[t]-\bmod _{\mathbf{z}}$. In geometric language, we have that $R$ is an algebra object in $\mathrm{QC}\left(\mathbb{A}^{1} / \mathbb{G}_{m}\right)$, and $R$-modz consists of $R$-module objects in $\mathrm{QC}\left(\mathbb{A}^{1} / \mathbb{G}_{m}\right)$. 
Definition 2.7. The periodic localization $R$ - $\bmod _{p e r}$ is the stable $\infty$-category defined by the following equivalent constructions.

(1) Geometric formulation: let $p t=\mathbb{G}_{m} / \mathbb{G}_{m} \subset \mathbb{A}^{1} / \mathbb{G}_{m}$ be the standard open embedding. We define $R$-mod $p e r$ to be the base change

$$
R-\bmod _{p e r}=R-\bmod _{\mathbf{z}} \otimes_{\mathrm{QC}\left(\mathbb{A}^{1} / \mathbb{G}_{m}\right)} \mathrm{QC}(p t) .
$$

(2) Algebraic formulation: in the language of $\mathbf{Z}$-graded rings, the above formulation (1) translates to the base change

$$
R-\bmod _{p e r}=R-\bmod _{\mathbf{z}} \otimes_{k[t]-\bmod _{\mathbf{z}}} k\left[t, t^{-1}\right]-\bmod _{\mathbf{z}} .
$$

(3) Formulation in terms of generator: standard adjunctions show that the above formulations (1) and (2) are equivalent to the stable $\infty$-category of modules for the localized algebra

$$
R-\bmod _{p e r}=R \otimes_{k[t]} k\left[t, t^{-1}\right]-\bmod _{\mathbf{z}} .
$$

In the definition, the tensor products in formulations (1) and (2) are calculated in the $\infty$ category of stable, presentable $\infty$-categories. Note that the periodic localization is no longer $\mathbf{Z}$-graded since it results from the base change to a point.

For $R$ an arbitrary algebra object in $k[t]-\bmod _{\mathbf{z}}$, it will also be useful to speak of the periodic localization for the shear shifted algebra $R_{[2 n]}$. Note that $R_{[2 n]}$ is naturally an algebra object in $k[t]_{[2 n]}-\bmod _{\mathbf{z}}$, and that $k[t]_{[2 n]}=k[v]$ where $v$ has degree $-2 n$ and weight 1 . We can then form the periodic localization

$$
R_{[n]}-\bmod _{p e r}=R-\bmod _{\mathbf{z}} \otimes_{k[v]-\bmod _{\mathbf{z}}} k\left[v, v^{-1}\right]-\bmod _{\mathbf{z}}
$$

and the equivalence of Proposition 2.6 induces an equivalence

$$
R_{[n]}-\bmod _{p e r} \simeq R-\bmod _{p e r} .
$$

2.3.3. Sheafified version. It is worthwhile to explain a version of the preceding discussion in a sheafified context.

Fix a derived stack $X$ with stable $\infty$-category $\mathrm{QC}(X)$ of quasicoherent sheaves. Suppose $R_{X}$ is a $\mathbf{Z}$-graded algebra object in the monoidal $\infty$-category of endofunctors of $\mathrm{QC}(X)$. Then we have the following sheafified versions of the preceding constructions.

We write $R_{X}$ - $\bmod _{\mathbf{z}}$ for the stable $\infty$-category of $\mathbf{Z}$-graded $R_{X}$-modules in $\mathrm{QC}(X)$. When $R_{X}$ is concentrated in nonnegative weights, we write $\widehat{R}_{X}$ for the completion of $R_{X}$ with respect to the positive weight direction. We write $\widehat{R}_{X}-\operatorname{cmod}_{\mathbf{z}}$ for the stable $\infty$-category of compatibly $\mathbf{Z}$-graded complete $\widehat{R}_{X}$-modules.

For $n \in \mathbf{Z}$, we write $R_{X,[n]}$ for the algebra defined by the shear shifting

$$
R_{X,[n]}=\oplus_{i \in \mathbf{Z}} R_{X}^{i}[n i] .
$$

There is a canonical weight-preserving equivalence of stable $\infty$-categories

$$
R_{X}-\bmod _{\mathbf{z}} \simeq R_{X,[n]}-\bmod _{\mathbf{z}} .
$$

Finally, consider once again the $\mathbf{Z}$-graded polynomial algebra $k[t]$ with $t$ of degree 0 and weight 1 so that we have the affine line $\mathbb{A}^{1}=\operatorname{Spec} k[t]$ with the standard $\mathbb{G}_{m}$-action. Via the correspondence $X \times\left(\mathbb{A}^{1} / \mathbb{G}_{m}\right) \leftarrow X \times \mathbb{A}^{1} \rightarrow X$, we can view the stable $\infty$-category $\mathrm{QC}(X \times$ $\left.\left(\mathbb{A}^{1} / \mathbb{G}_{m}\right)\right)$ as $\mathbf{Z}$-graded modules in $\mathrm{QC}(X)$ over the constant $\mathbf{Z}$-graded algebra object $\mathcal{O}_{X \times \mathbb{A}^{1}} \simeq$ $\mathcal{O}_{X} \otimes_{k} k[t]$. In turn, we can view $\mathcal{O}_{X} \otimes_{k} k[t]$ as a $\mathbf{Z}$-graded algebra object in the monoidal $\infty$-category of endofunctors of $\mathrm{QC}(X)$. 
Now suppose $R_{X}$ is a $\mathbf{Z}$-graded $\mathcal{O}_{X} \otimes_{k} k[t]$-algebra object in the monoidal $\infty$-category of endofunctors of $\mathrm{QC}\left(X \times \mathbb{A}^{1}\right)$. Then we have the periodic localization of $R_{X}-\bmod _{\mathbf{Z}}$ defined by the base change

$$
R_{X}-\bmod _{\text {per }}=R_{X}-\bmod _{\mathbf{z}} \otimes_{k[t]-\bmod _{\mathbf{z}}} k\left[t, t^{-1}\right]-\bmod _{\mathbf{z}} .
$$

It also admits the two alternative formulations discussed above. Similarly, we have the periodic localization for the shear shifted algebra

$$
R_{X,[n]}-\bmod _{p e r}=R_{X}-\bmod _{\mathbf{z}} \otimes_{k[v]-\bmod \mathbf{z}} k\left[v, v^{-1}\right]-\bmod _{\mathbf{z}}
$$

where $v$ has degree $-2 n$ and weight 1 , and it satisfies the compatibility

$$
R_{X,[n]}-\bmod _{p e r} \simeq R_{X}-\bmod _{p e r} .
$$

\section{Affinization}

In this section, we study the relationship between a derived stack $X$ and its commutative ring of derived global functions $\mathcal{O}(X)$. We continue with $k$ a fixed discrete commutative rational algebra.

3.1. Background. Recall that $A l g_{k}$ denotes the category of discrete commutative $k$-algebras, so its opposite category $\mathrm{Alg}_{k}^{o p}$ is the category of affine schemes over $k$. Let $S c h e m e_{k}$ denote the category of all schemes over $k$. Recall the standard adjoint pair of functors

$$
\mathcal{O}: \text { Scheme }_{k} \longleftrightarrow \text { Alg }_{k}^{o p}: \text { Spec }
$$

where $\mathcal{O}$ is the global functions functor, and Spec is the inclusion of affine schemes into all schemes. The functor of affinization is the monad

$$
\text { Aff }=\operatorname{Spec} \mathcal{O}: \text { Scheme }_{k} \rightarrow \text { Scheme }_{k}
$$

that sends a scheme $X$ to the spectrum of its ring of global functions $\mathcal{O}(X)$. In particular, for any scheme $X$, there is an affine scheme $\operatorname{Aff}(X)$ and a universal map $X \rightarrow \operatorname{Aff}(X)$ through which we can factor any map from $X$ to an affine scheme.

Now let $D G A_{k}^{+}$denote the $\infty$-category of coconnective commutative dg $k$-algebras. topological space $K$, we can assign its $k$-valued cochains $C^{*}(K, k)$ as an object of $D G A_{k}^{+}$. More generally, to any derived stack $X$, we can assign its global functions $\mathcal{O}(X)$, also known as the coherent cohomology of the structure sheaf, as a (not necessarily connective or coconnective) commutative algebra

$$
X=\operatorname{colim} \operatorname{Spec} R_{\alpha}, \quad R_{\alpha} \in D G A_{k}^{-}, \quad \mathcal{O}(X)=\lim R_{\alpha} \in D G A_{k} .
$$

(More precisely, we are glossing over set theoretic issues, which can be dealt with by requiring standard smallness conditions on our stacks, see e.g. HAG1.) If $X$ is an ordinary stack (for example, an ordinary scheme), then $\mathcal{O}(X) \in D G A_{k}^{+}$, while if $X$ is an affine derived scheme, then $\mathcal{O}(X) \in D G A_{k}^{-}$.

In [To1], Toën introduces affine stacks as the full subcategory of underived stacks represented by the opposite category $\left(D G A_{k}^{+}\right)^{o p}$ of coconnective commutative $k$-algebras 2 In particular, the affine stack corresponding to $C^{*}(K, k)$ is the algebraic avatar of the $k$-linear homotopy theory of $K$.

\footnotetext{
${ }^{2} \mathrm{~A}$ word of caution: the embedding of $\left(D G A_{k}^{+}\right)^{o p}$ into stacks does not preserve colimits, so it is not recommended to think of affine stacks as simplicial affine schemes as one often thinks of stacks.
} 
3.2. Affine derived stacks. We will be interested in the analogue of affinization for derived stacks, and thus we would like to consider the larger class of "affine derived stacks" corresponding to all (not neccessarily coconnective) commutative dg $k$-algebras. To do so, we introduce the functor

$$
\text { Spec : } D G A_{k}^{o p} \rightarrow D S t, \quad \operatorname{Spec}(R)(A)=\operatorname{Hom}_{D G A_{k}}(R, A), \quad A \in D G A_{k}^{-} .
$$

In other words, Spec assigns to $R \in D G A_{k}^{o p}$ the functor it represents when restricted to connective commutative dg $k$-algebras (which is automatically a stack, since it distributes over limits in $\left.D G A_{k}^{-}\right)$.

Proposition 3.1. There is a natural adjunction

$$
\mathcal{O}: D S t_{k} \longleftrightarrow D G A_{k}^{o p}: \mathrm{Spec}
$$

Proof. Note first that $\mathcal{O}$ is colimit preserving, and so by the adjoint functor theorem possesses a right adjoint. We will identify this adjoint with Spec by testing maps into Spec. Let $R \in$ $D G A_{k}$, and let $A \in D G A_{k}^{-}$. By definition of the functor $\operatorname{Spec} R$, maps of derived stacks Spec $A \rightarrow \operatorname{Spec} R$ are given by maps of $\operatorname{dg} k$-algebras $R \rightarrow A$. Using this, we establish the desired adjunction as follows. Consider any $X \in D S t$, and write $X=\operatorname{colim} \operatorname{Spec} A_{i}$. Then we have equivalences

$$
\begin{aligned}
\operatorname{Hom}_{D S t}(X, \operatorname{Spec} R) & \simeq \operatorname{Hom}_{D S t}\left(\operatorname{colim} \operatorname{Spec} A_{i}, \operatorname{Spec} R\right) \\
& \simeq \lim \operatorname{Hom}_{D S t}\left(\operatorname{Spec} A_{i}, \operatorname{Spec} R\right) \\
& \simeq \lim \operatorname{Hom}_{D G A_{k}}\left(R, A_{i}\right) \\
& \simeq \operatorname{Hom}_{D G A_{k}}\left(R, \lim A_{i}\right) \\
& \simeq \operatorname{Hom}_{D G A_{k}^{o p}}(\mathcal{O}(X), R) .
\end{aligned}
$$

Definition 3.2. We call the endofunctor

$$
\text { Aff : } D S t_{k} \rightarrow D S t_{k} \quad \operatorname{Aff}(X)=\operatorname{Spec} \mathcal{O}(X)
$$

the affinization functor. We refer to derived stacks $X$ for which the canonical morphism $X \rightarrow$ $\operatorname{Aff}(X)$ is an equivalence as affine derived stacks.

Note that Aff has a canonical monad structure, and any morphism from $X$ to an affine derived stack factors through the canonical morphism $X \rightarrow \operatorname{Aff}(X)$.

We will only use the above constructions in the following specific setting. We will consider morphisms $Y \rightarrow \operatorname{Spec} A$, where $Y$ is a topological space, and $A \in D G A_{k}^{-}$so that $\operatorname{Spec} A$ is an affine derived scheme. Then by adjunction, the original morphism canonically factors into morphisms

$$
Y \longrightarrow \operatorname{Aff}(Y)=\operatorname{Spec} \mathcal{O}(Y) \longrightarrow \operatorname{Spec}(\mathcal{O}(\operatorname{Spec} A)) \simeq \operatorname{Spec} A .
$$

Note that we need the generality of affine derived stacks introduced above since we need the adjunction simultaneously for $\mathcal{O}(Y) \in D G A_{k}^{+}$as well as $A \in D G A_{k}^{-}$.

Remark 3.3. We would like to thank Toën for pointing out that the derived version of Spec is not faithful. For example, Spec of the formal commutative dg alegbra $k\left[u, u^{-1}\right]$, with $|u|=2$, defines the empty stack: there are no morphisms from $k\left[u, u^{-1}\right]$ to a connective commutative dg algebra since $u$ is invertible but would need to vanish under such a morphism. 
3.3. Affinization of groups. We would like to understand the impact of affinization on group derived stacks and their actions on other derived stacks. We will develop a minimum of theory to apply to the group stack $S^{1}$. All we will use is that $S^{1}$ is a finite CW complex so that we can appeal to the following immediate lemma.

Lemma 3.4. Let $\pi: X \rightarrow$ Spec $k$ be a finite $C W$ complex regarded as a derived stack. The global sections functor $\pi_{*}: \mathrm{QC}(X) \rightarrow \operatorname{Mod}_{k}$ preserves colimits (the structure sheaf $\mathcal{O}_{X}$ is a compact object), and the pullback functor $\pi^{*}: \operatorname{Mod}_{k} \rightarrow \mathrm{QC}(X)$ preserves limits (global functions $\mathcal{O}(X)$ form a dualizable $k$-module). The same assertions hold for the affinization $\pi^{\prime}: \operatorname{Aff}(X) \rightarrow \operatorname{Spec} k$.

Proof. For $X$, both assertions follow from the fact that $X$ is a finite colimit of copies of Spec $k$. For $\operatorname{Aff}(X)$, consider the natural morphism $\alpha: X \rightarrow \operatorname{Aff}(X)$, and observe that $\pi_{*}^{\prime} \simeq \pi_{*} \alpha^{*}$ and $\pi^{\prime *} \simeq \alpha_{*} \pi^{*}$.

Definition 3.5. We define the full $\infty$-subcategory $D S t_{k}^{f c} \subset D S t_{k}$ of functionally compact derived stacks to consist of derived stacks $X$ whose structure sheaf $\mathcal{O}_{X}$ is a compact object of $\mathrm{QC}(X)$, or in other words, for which the functor of global sections $\Gamma: \mathrm{QC}(X) \rightarrow \operatorname{Mod}_{k}$ preserves colimits.

Thus the above lemma shows that finite $\mathrm{CW}$ complexes are functionally compact. The following technical assertion motivates the first conclusion of the lemma (considerations of descent will motivate the second conclusion).

Proposition 3.6. BFN, Proposition 3.10, see also Remark 3.11] Suppose $X, Y \rightarrow$ Spec $k$ are functionally compact stacks. Then morphisms $f: X \rightarrow Y$ satisfy the projection formula:

$$
f_{*}\left(f^{*} M \otimes_{\mathcal{O}_{X}} N\right) \simeq M \otimes_{\mathcal{O}_{Y}} f_{*} N, \quad M \in \mathrm{QC}(Y), N \in \mathrm{QC}(X) .
$$

Furthermore, if $g: \tilde{Y} \rightarrow Y$ is any map of derived stacks, the resulting base change map $g^{*} f_{*} \rightarrow$ $\tilde{f}_{*} \tilde{g}^{*}$ is an equivalence.

Proposition 3.7. The restriction of the affinization functor Aff to functionally compact stacks is naturally monoidal.

Proof. For $X, Y$ functionally compact, the projection formula provides a canonical equivalence

$$
\mathcal{O}(X \times Y) \simeq \mathcal{O}(X) \otimes \mathcal{O}(Y)
$$

Thus affinization commutes with finite products of functionally compact stacks, and hence by [L2, Corollary 2.4.1.9], it naturally defines a monoidal functor.

Corollary 3.8. For a group $G$ in finite $C W$ complexes, its affinization $\operatorname{Aff}(G)$ inherits a canonical structure of group derived stack, and the affinization map $G \rightarrow \operatorname{Aff}(G)$ inherits the structure of a group homomorphism.

Proof. Group objects are defined by diagrams involving solely the monoidal structure.

In what remains of this section, we consider the role of affinization in understanding $G$-actions on affine derived schemes. We begin with the linear case of $G$-actions on plain modules.

3.3.1. G-actions on modules. Given a group derived stack $G$, we have the natural derived stack $B G$ given by the usual classifying space construction. As usual, it comes equipped with a universal $G$-bundle $\pi$ : Spec $k=E G \rightarrow B G$.

Definition 3.9. We define the $\infty$-category of $k$-modules with $G$-action to be the $\infty$-category of quasicoherent sheaves $\mathrm{QC}(B G)$. We say that a $k$-module $M$ is equipped with a $G$-action if we are given $\mathcal{M} \in \mathrm{QC}(B G)$ with an identification $\pi^{*} \mathcal{M} \simeq M$. 
Lemma 3.10. Let $G$ be a group in finite $C W$ complexes. There is a canonical $k$-linear equivalence

$$
\mathrm{QC}(B G) \simeq \mathcal{O}(G) \text {-comod }
$$

identifying the structures of $G$-action and $\mathcal{O}(G)$-comodule on $k$-modules. The same assertion holds for the affinization $\operatorname{Aff}(G)$.

Proof. The assertion is a standard descent argument. Namely, consider the usual $k$-linear adjunction

$$
\pi^{*}: \mathrm{QC}(B G) \longleftrightarrow \mathrm{QC}(\operatorname{Spec} k): \pi_{*}
$$

associated to the map $\pi: \operatorname{Spec} k=E G \rightarrow B G$. It provides a natural $k$-linear morphism

$$
\tilde{\pi}^{*}: \mathrm{QC}(B G) \longrightarrow \pi^{*} \pi_{*} \text {-comod }
$$

which we seek to show is a $k$-linear equivalence. Recall that a $k$-linear equivalence is nothing more than a $k$-linear functor which is an equivalence on the underlying plain $\infty$-categories. Thus we can forget the $k$-linear structure and simply show that $\tilde{\pi}^{*}$ is an equivalence.

Observe that the above adjunction satisfies the criteria of the comonadic form of Lurie's Barr-Beck theorem. Namely, $\pi^{*}$ is conservative, and preserves limits by Lemma 3.4. Moreover, we may invoke Proposition 3.6, and by repeated base change in the diagram

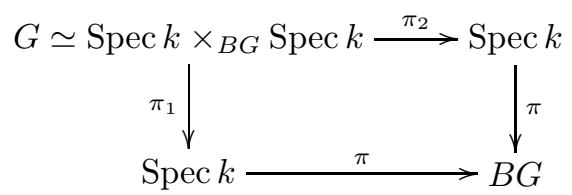

see that the comonad $\pi^{*} \pi_{*}$ is equivalent to tensoring with the coalgebra

$$
\pi^{*} \pi_{*} \mathcal{O}_{\text {Spec } k} \simeq \pi_{2 *} \pi_{1}^{*} \mathcal{O}_{\text {Spec } k} \simeq \mathcal{O}(G) .
$$

The proof for $\operatorname{Aff}(G)$ is exactly the same.

Recall that the canonical morphism $G \rightarrow \operatorname{Aff}(G)$ is a group homomorphism, and hence the induced identification $\mathcal{O}(G) \simeq \mathcal{O}(\operatorname{Aff}(G))$ respects coalgebra structures.

Corollary 3.11. Let $G$ be a group in finite $C W$ complexes. Then pullback along the canonical map $G \rightarrow \operatorname{Aff}(G)$ provides an equivalence from the $\infty$-category of $k$-modules with $\operatorname{Aff}(G)$-action to that of $k$-modules with $G$-action.

3.3.2. G-actions on affine derived schemes. Now we consider the nonlinear setting of $G$-actions on affine derived schemes.

Proposition 3.12. Let $G$ be a functionally compact group derived stack. Then pullback along the canonical map $G \rightarrow \operatorname{Aff}(G)$ provides an equivalence from the $\infty$-category of affine derived schemes with $\operatorname{Aff}(G)$-action to that of affine derived schemes with $G$-action.

Proof. Note that the structure sheaf of an affine derived scheme is a compact object. Since the affinization functor Aff restricted to the $\infty$-category of derived stacks with compact structure sheaf is monoidal, it takes module objects for $G$ to module objects for $\operatorname{Aff}(G)$. For $X$ an affine derived scheme with $G$-action, it follows that $X=\operatorname{Aff}(X)$ carries a canonical $\operatorname{Aff}(G)$-action. Moreover, the identity map $X \rightarrow \operatorname{Aff}(X)=X$ intertwines the $G$ and $\operatorname{Aff}(G)$-actions on $X$. Thus the $G$-action on $X$ factors through the corresponding $\operatorname{Aff}(G)$-action (equivalently, the action of the kernel of $G \rightarrow \operatorname{Aff}(G)$ is trivialized). This provides an inverse construction to pullback along the map $G \rightarrow \operatorname{Aff}(G)$. 
It is worth noting that we can transport via Lemma 3.10 the symmetric monoidal structure on the $\infty$-category $\mathrm{QC}(B G)$ to one on the $\infty$-category $\mathcal{O}(G)$-comod. With this in mind, we can restate the above proposition as saying that there is a canonical equivalence between the $\infty$-category of affine derived schemes with $G$-action and the opposite of the $\infty$-category of connective commutative ring objects in $\mathcal{O}(G)$-comodules.

3.4. Application: circle actions. Let's apply the preceding to the circle $S^{1}=B \mathbf{Z}=$ $K(\mathbf{Z}, 1) \in \mathcal{S}$ considered as a derived stack. It is a group object [L1, 7.2.2.1] in spaces, and thus also in derived stacks. Its algebra of cochains is given explicitly as follows:

$$
\mathcal{O}\left(S^{1}\right)=C^{*}\left(S^{1}, k\right) \simeq H^{*}\left(S^{1}, k\right)=k[\eta] /\left(\eta^{2}\right), \quad|\eta|=1 .
$$

We can think of this algebra as a graded version of the dual numbers, and so think of maps out of the affinization $\operatorname{Aff}\left(S^{1}\right)$ as giving "tangents" of degree -1 . The ring $\mathcal{O}\left(S^{1}\right)$ is the free commutative $k$-algebra (in the graded sense) on a single generator of degree one (recall that we always work rationally). Thus its spectrum $\operatorname{Aff}\left(S^{1}\right)$ is the classifying stack of the additive group or shifted affine line.

Lemma 3.13. The affinization morphism is an equivalence of group derived stacks

$$
\operatorname{Aff}\left(S^{1}\right) \stackrel{\sim}{\longrightarrow} B \mathbb{G}_{a}=K\left(\mathbb{G}_{a}, 1\right)=\mathbb{A}^{1}[1] .
$$

Proof. The canonical homomorphism $\mathbf{Z} \rightarrow \mathbb{G}_{a}$ induces a homomorphism of group stacks $B \mathbf{Z} \rightarrow$ $B \mathbb{G}_{a}$. The corresponding pullback on cochains is an equivalence, and so the induced group homomorphism $\operatorname{Aff}(B \mathbf{Z}) \rightarrow B \mathbb{G}_{a}$ is an equivalence. Hence the morphism $B \mathbf{Z} \rightarrow B \mathbb{G}_{a}$ agrees with the affinization morphism for $S^{1} \simeq B \mathbf{Z}$.

The formality of the cochain algebra $\mathcal{O}\left(S^{1}\right) \simeq H^{*}\left(S^{1}, k\right)$ implies that $\operatorname{Aff}\left(S^{1}\right) \simeq B \mathbb{G}_{a}$ inherits a canonical action of the multiplicative group $\mathbb{G}_{m}$ induced by its grading action on $H^{*}\left(S^{1}, k\right)$. This will be a crucial structure in our analysis of sheaves on loop spaces. We fix conventions so that $\mathbb{G}_{m}$ acts trivially on the subspace $H^{0}\left(S^{1}, k\right)$, and rescales the subspace $H^{1}\left(S^{1}, k\right)$ with weight one. Note that under the identification $\operatorname{Aff}\left(S^{1}\right) \simeq B \mathbb{G}_{a}$, the $\mathbb{G}_{m}$-action determined by formality is concretely given by the usual scaling $\mathbb{G}_{m}$-action on $\mathbb{G}_{a}$.

3.4.1. Circle actions on modules and mixed complexes. We will denote the homology, or "group algebra", of the circle by

$$
\boldsymbol{\Lambda}=H_{-*}\left(S^{1}, k\right)=k[\lambda] /\left(\lambda^{2}\right), \quad|\lambda|=-1 .
$$

With our conventions, $H_{-1}\left(S^{1}, k\right)$ has $\mathbb{G}_{m}$-weight grading -1 . Convolution equips $\boldsymbol{\Lambda}$ with a commutative algebra structure dual to the coalgebra structure on $\mathcal{O}\left(S^{1}\right)=H^{*}\left(S^{1}, k\right)$. Thus $\mathcal{O}\left(S^{1}\right)$-comodules are the same thing as $\boldsymbol{\Lambda}$-modules. Note that the differential graded notion or more relaxed $A_{\infty}$-notion of $\boldsymbol{\Lambda}$-module are equivalent, and so there is no risk of ambiguity in our terminology. Thus Corollary 3.11 identifies circle actions on modules with square zero endomorphisms of degree -1 , or so-called mixed complexes.

Corollary 3.14. The $\infty$-category of $k$-modules with $S^{1}$-action is equivalent to that of differential graded $\boldsymbol{\Lambda}$-modules (so-called mixed complexes).

This is a well known story in other contexts, see [Lo, Kas, DK]. The Borel homotopy theory of $S^{1}$-actions (or homotopy theory over $B S^{1}$ ) is often modelled using the theory of cyclic objects. In particular, the $\infty$-category of $k$-modules with $S^{1}$-action is the Dwyer-Kan simplicial localization of the model category of cyclic $k$-modules. The above corollary is the (localized) $\infty$-categorical version of the result of Dwyer-Kan [DK] giving a Quillen equivalence of model categories between $\boldsymbol{\Lambda}$-modules and cyclic $k$-modules. 
3.4.2. Circle actions on affine derived schemes. We have seen that group actions on affine derived schemes are the same thing as actions of their affinizations. We have also seen that the latter are equivalent to connective commutative ring objects in comodules over the group coalgebra. Applying this to the circle, and using the duality of the the group coalgebra with the group algebra, we have the following.

Corollary 3.15. The $\infty$-category of affine derived schemes with $S^{1}$-action is equivalent to that of affine derived schemes with $B \mathbb{G}_{a}$-action.

It is worth noting that we can transport via Lemma 3.10 the symmetric monoidal structure on the $\infty$-category $\mathrm{QC}\left(B S^{1}\right)$ to one on the $\infty$-category of differential graded $\boldsymbol{\Lambda}$-modules. With this in mind, we can restate the above corollary as saying that there is a canonical equivalence between the $\infty$-category of affine derived schemes with $S^{1}$-action and the opposite of the $\infty$ category of connective commutative ring objects in mixed complexes.

Remark 3.16 (Hopf algebras and formality). An important result of Toën and Vezzosi [ToVe5] establishes the formality of $\mathcal{O}\left(S^{1}\right)$ as a differential graded Hopf algebra. Such formality implies that the symmetric monoidal $\infty$-category of mixed complexes can be modelled concretely by the obvious tensor product structure on cochain complexes with a square zero endomorphism of degree -1 . In particular, algebra objects therein can be modelled concretely by algebras with a square zero derivation of degree -1 . We will see that when it comes to the loop space of an affine derived scheme, using the additional structure of weights, one can easily arrive at such a description of the $S^{1}$-action on the algebra of functions.

3.5. Unwinding higher coherences. Let us return to the general setting of a group derived stack $G$ acting on a derived stack $X$. Recall that this involves an action map $G \times X \rightarrow X$ along with higher coherent structures reflecting that it is a group action. We would like to investigate what information is contained in the action map alone, and what is contained in the higher coherent structures.

Let $\mathcal{S}_{*}, D S t_{*}$ denote pointed spaces and pointed derived stacks, respectively. Recall the familiar suspension-loop adjunction

$$
\Sigma: \mathcal{S}_{*} \longleftrightarrow \mathcal{S}_{*}: \Omega
$$

We continue to use the same notation for its (target-wise) extension to $D S t_{*}$.

To any $Y \in \mathcal{S}_{*}$, we have the free monoid monad

$$
F(Y)=\Omega \Sigma Y \in \mathcal{S}_{*},
$$

(which is in fact a derived group object) along with a canonical map $Y \rightarrow F(Y)$. (This is equivalent to the familiar James construction of the free monoid on a space.)

We now consider $Y$ and $F(Y)$ as pointed derived stacks. By adjunction, any candidate action map $\alpha_{Y}: Y \times X \rightarrow X$ in $D S t_{*}$ canonically extends to a $F(Y)$-action on $X$ such that the restriction of the action map $\alpha_{F(Y)}: F(Y) \times X \rightarrow X$ along the canonical map $Y \rightarrow$ $F(Y)$ recovers the original map $\alpha_{Y}$. In other words, any map of derived stacks $Y \rightarrow \operatorname{Aut}(X)$ canonically extends to a group homomorphism $F(Y) \rightarrow \operatorname{Aut}(X)$ from the free group such that the composition $Y \rightarrow F(Y) \rightarrow \operatorname{Aut}(X)$ is the original map. Conversely, any $F(Y)$-action on $X$ is determined by the restriction of its action map $\alpha_{F(Y)}: F(Y) \times X \rightarrow X$ along the canonical map $Y \rightarrow F(Y)$. In other words, any group homomorphism $F(Y) \rightarrow \operatorname{Aut}(X)$ from the free group is determined by the composition $Y \rightarrow F(Y) \rightarrow \operatorname{Aut}(X)$.

Now suppose we apply the above constructions when $Y$ is a group derived stack $G$ pointed by the identity $p t \rightarrow G$. Then we not only have the canonical map $G \rightarrow F(G)$, but also a canonical group homomorphism $\pi: F(G) \rightarrow G$ realizing $G$ as a "quotient" of a free group. Let 
us denote by $K$ the kernel (or homotopy fiber above the identity) of the homomorphism $\pi$ so that we have a pullback diagram of groups

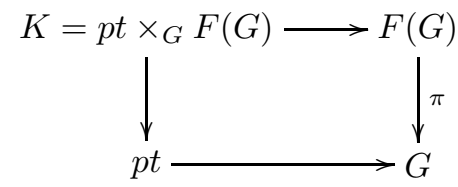

Suppose further that we have a $G$-action on a derived stack $X$. So in particular, by restriction along $\pi$, we have a $F(G)$-action on $X$. The $F(G)$-action on $X$ is equivalent to the action map $G \times X \rightarrow X$ alone, while $K$ automatically acts trivially. Conversely, given an $F(G)$-action, the action of $K$ is the first obstruction for the action to descend to $G$. In the classical setting of group actions on sets, this is the only obstruction, measuring the failure of the action map on $G$ to satisfy associativity. In the homotopical setting, however, there are higher obstructions (since the above diagram is not necessarily a pushout diagram).

3.5.1. Unwinding circle actions. Next let's specialize to the case when $G$ is the circle $S^{1}$. Applying the above constructions, and the identity $\Sigma S^{1} \simeq S^{2}$ so that $F\left(S^{1}\right) \simeq \Omega S^{2}$, we obtain the pullback diagram of groups

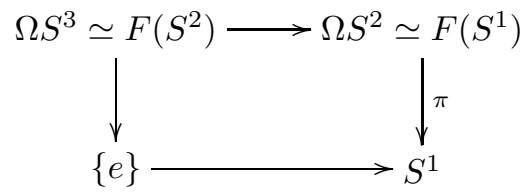

This is nothing more than a rotation of the familiar Hopf fibration $S^{3} \rightarrow S^{2}$. More precisely, we can obtain the above diagram by applying the limit preserving functor $\Omega$ to the classifying map of the Hopf fibration

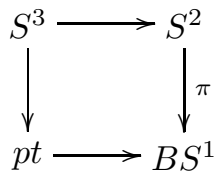

Recall that an action of the free group $\Omega S^{2} \simeq F\left(S^{1}\right)$ on a space $X$ amounts to a pointed map $S^{1} \times X \rightarrow X$. Similarly, since the kernel $\Omega S^{3} \simeq F\left(S^{2}\right)$ is a free group as well, its action on a space $X$ amounts to a pointed map $S^{2} \times X \rightarrow X$.

It is also clear from this picture that an $\Omega S^{2}$-action with a trivialization of the induced $\Omega S^{3}$-action does not amount to an $S^{1}$-action. More precisely, an $\Omega S^{2}$-space is a space over $S^{2} \simeq \mathbf{C P}^{1} \subset \mathbf{C P}^{\infty} \simeq B S^{1}$, and trivializing the induced $\Omega S^{3}$-action means extending the map to only $\mathbf{C P}^{2} \subset \mathbf{C P}{ }^{\infty}$ with an infinite sequence of higher obstructions remaining to overcome.

3.5.2. Loop space actions. Finally, we give an algebraic description of $\Omega X$-actions. In preceding sections, we have made technical use of when a group $G$ is a finite $\mathrm{CW}$ complex. Now we will make use of the assumption that the space $X$ in which we consider loops is a finite CW complex.

Lemma 3.17. Let $X$ be a finite, simply-connected $C W$ complex. There is a canonical $k$-linear equivalence

$$
\mathrm{QC}(X) \simeq \mathcal{O}(X)-\bmod
$$

identifying sheaves with modules over global functions. The same assertion holds for the affinization $\operatorname{Aff}(X)$. 
Proof. The assertion is a standard ascent argument. Namely, consider the usual $k$-linear adjunction

$$
\pi^{*}: \mathrm{QC}(\operatorname{Spec} k) \longleftrightarrow \mathrm{QC}(X): \pi_{*}
$$

associated to the map $\pi: X \rightarrow \operatorname{Spec} k$. It provides a natural $k$-linear morphism

$$
\tilde{\pi}_{*}: \mathrm{QC}(X) \longrightarrow \pi_{*} \pi^{*}-\bmod
$$

which we seek to show is a $k$-linear equivalence. Recall that a $k$-linear equivalence is nothing more than a $k$-linear functor which is an equivalence on the underlying plain $\infty$-categories. Thus we can forget the $k$-linear structure and simply show that $\tilde{\pi}^{*}$ is an equivalence.

Observe that the above adjunction satisfies the criteria of the monadic form of Lurie's BarrBeck theorem. Namely, $\pi_{*}$ is conservative (here we use that $X$ is simply connected), and preserves colimits by Lemma 3.4. Moreover, we may invoke Proposition 3.6. and by repeated use of the projection formula see that the monad $\pi_{*} \pi^{*}$ is equivalent to tensoring with the algebra

$$
\pi_{*} \pi^{*} \mathcal{O}_{\text {Spec } k} \simeq \mathcal{O}(X) .
$$

The proof for $\operatorname{Aff}(X)$ is exactly the same.

Recall that an $\Omega X$-module is simply an object of $\mathrm{QC}(B \Omega X)$, and that when $X$ is connected, there is an equivalence $B \Omega X \simeq X$.

Corollary 3.18. Let $X$ be a finite, connected, simply-connected $C W$ complex.

(1) The $\infty$-category of $k$-modules with $\Omega X$-action is equivalent to that of $\mathcal{O}(X)$-modules.

(2) The $\infty$-category of affine derived schemes with $\Omega X$-action is equivalent to the opposite of the $\infty$-category of connective commutative ring objects in the symmetric monoidal $\infty$-category of $\mathcal{O}(X)$-modules.

\section{LOOPS IN DERIVED SCHEMES}

Now we turn to loop spaces, in particular loop spaces of derived schemes. We continue to work over a fixed commutative $\mathbf{Q}$-algebra $k$. By a derived scheme, we will always mean a quasi-compact derived scheme with affine diagonal. In particular, one could work with the assumption that the derived scheme is quasi-compact and separated.

4.1. Loops and tangents. Recall that we think of the circle $S^{1}$ as a derived stack, and we have identified its affinization with the classifying stack $B \mathbb{G}_{a}$ of the additive group.

Definition 4.1. The loop space of a derived stack $X$ is the derived mapping stack

$$
\mathcal{L} X=\operatorname{Map}_{D S t}\left(S^{1}, X\right) .
$$

If we realize $S^{1}$ by two 0 -simplices connected by two 1 -simplices, we obtain a concrete model for the loop space as a fiber product of derived stacks along diagonal maps

$$
\mathcal{L} X \simeq X \times_{X \times X} X \text {. }
$$

Informally speaking, $\mathcal{L} X$ consists of pairs of points of $X$ which are equal and then equal again. It follows immediately that if $X$ has affine diagonal (in particular for quasicompact quasiseparated derived schemes), then $\mathcal{L} X$ is the relative spectrum of Hochschild chains

$$
\mathcal{L} X \simeq \operatorname{Spec}_{\mathcal{O}_{X}}\left(\mathcal{O}_{X} \otimes S^{1}\right)=\operatorname{Spec}_{\mathcal{O}_{X}}\left(\mathcal{O}_{X} \otimes_{\mathcal{O}_{X} \otimes \mathcal{O}_{X}} \mathcal{O}_{X}\right) .
$$

Thanks to the following simple observation, the case of derived schemes (always quasicompact with affine diagonal) is no more difficult than that of affine derived schemes. 
Lemma 4.2. For $X$ a derived scheme, and $U \rightarrow X$ a Zariski open, the induced map $\mathcal{L} U \rightarrow \mathcal{L} X$ is also Zariski open. The assignment of loops

$$
U \longmapsto \mathcal{L} U
$$

forms a cosheaf on the Zariski site of $X$.

Proof. Recall that $\mathcal{L} X$ is the relative spectrum of Hochschild chains of $X$. For $u: U \rightarrow X$ a Zariski open affine, thanks to the projection formula and the obvious adjunction identity

$$
u^{*} u_{*} \mathcal{O}_{U} \simeq \mathcal{O}_{U} \otimes \mathcal{O}_{X} \mathcal{O}_{U} \stackrel{\sim}{\longrightarrow} \mathcal{O}_{U},
$$

we have an equivalence of $\mathcal{O}_{X}$-algebras

$$
u^{*} \mathcal{O}_{\mathcal{L} X}=\mathcal{O}_{\mathcal{L} X} \otimes_{\mathcal{O}_{X}} \mathcal{O}_{U} \simeq\left(\mathcal{O}_{X} \otimes_{\mathcal{O}_{X} \otimes \mathcal{O}_{X}} \mathcal{O}_{X}\right) \otimes_{\mathcal{O}_{X}} \mathcal{O}_{U} \stackrel{\sim}{\longrightarrow} \mathcal{O}_{U} \otimes_{\mathcal{O}_{U} \otimes \mathcal{O}_{U}} \mathcal{O}_{U} \simeq \mathcal{O}_{\mathcal{L} U}
$$

In other words, the natural maps form a Cartesian square

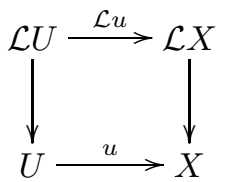

and since $u$ is Zariski open affine, $\mathcal{L} u$ is as well.

The cosheaf assertion follows immediately.

For an affine derived scheme $X=\operatorname{Spec} R$, since maps $S^{1} \rightarrow X$ factor through the affinization $S^{1} \rightarrow B \mathbb{G}_{a}$, we have an equivalence

$$
\mathcal{L} X \simeq \operatorname{Map}_{D S t}\left(B \mathbb{G}_{a}, X\right)
$$

intertwining the natural actions of $S^{1}$ and $B \mathbb{G}_{a}$. By Lemma 4.2, we see that the same holds true for any derived scheme.

Our immediate aim is to establish a more linear model for $\mathcal{L} X$. Recall that the cotangent complex of a derived $k$-algebra is a connective complex [L2, Corollary 7.4.3.6], and thus its shifted symmetric algebra $\Omega^{-\bullet}=\operatorname{Sym}^{\bullet} \Omega_{X}^{1}[1]$ is again connective. Thus we can consider $\Omega^{-\bullet}$ as a derived $\mathcal{O}_{X}$-algebra.

Definition 4.3. The odd tangent bundle of a derived stack $X$ is the linear derived stack

$$
\mathbb{T}_{X}[-1]=\operatorname{Spec}_{\mathcal{O}_{X}} \operatorname{Sym}^{\bullet}\left(\Omega_{X}[1]\right)
$$

where $\Omega_{X}$ is the cotangent complex of $X$.

When $X$ is an underived smooth scheme, we can use the Koszul resolution of the diagonal to calculate the Hochschild chain complex. The following is a generalization of the resulting Hochschild-Kostant-Rosenberg isomorphism [HKR] to arbitrary derived schemes.

Proposition 4.4. For $X$ a derived scheme, the loop space $\mathcal{L} X$ is identified with the odd tangent bundle $\mathbb{T}_{X}[-1]$ such that constant loops correspond to the zero section. Equivalently, we have an equivalence of $\mathcal{O}_{X}$-algebras

$$
\mathcal{O}_{X} \otimes_{\mathcal{O}_{X} \otimes \mathcal{O}_{X}} \mathcal{O}_{X} \simeq \Omega_{X}^{-\bullet} .
$$

Proof. By Lemma 4.2, it suffices to assume $X=\operatorname{Spec} R$ is affine. Then maps $S^{1} \rightarrow X$ factor through the affinization $S^{1} \rightarrow B \mathbb{G}_{a}$, and thus we have an equivalence

$$
\mathcal{L} X \simeq \operatorname{Map}_{D S t}\left(B \mathbb{G}_{a}, X\right) .
$$


Recall that for $S \in D G A_{k}^{-}$, the $S$-points of the derived stack $\operatorname{Map}_{D S t}\left(B \mathbb{G}_{a}, X\right)$ are the mapping space

$$
\operatorname{Map}_{D S t}\left(\operatorname{Spec} S \times B \mathbb{G}_{a}, X\right) \simeq \operatorname{Map}_{D S t}(\operatorname{Spec}(S \oplus S[-1]), X) \simeq \operatorname{Hom}_{D G A_{k}}(R, S \oplus S[-1]) .
$$

In general, for any $S$-module $M$, we denote by $S \oplus M$ the corresponding split square zero extension.

Next recall (see [HAG2, 1.4.1], L2, 7.4.1]) the universal property of the cotangent complex $\Omega_{R} \in \operatorname{Mod}_{R}$ as representing the functor of derivations. For any $S$-point $x: \operatorname{Spec} S \rightarrow X$, and any connective $S$-module $M$, we have a pullback diagram

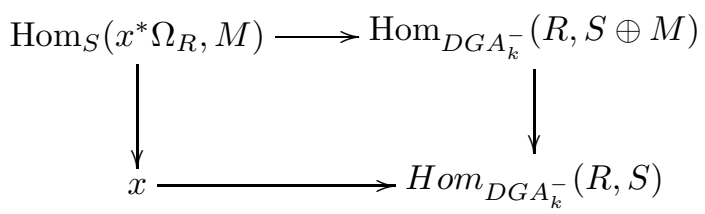

or in other words, an identification

$$
\operatorname{Hom}_{S}\left(x^{*} \Omega_{R}, M\right) \simeq \operatorname{Der}_{R}(S, M) .
$$

In fact this universal property of the cotangent complex holds without any connectivity assumptions, but we can impose connectivity as above thanks to the connectivity estimates of [L2, 7.4.3] (see also Remark 4.5). Thus we can take $M=S[-1]$ (although $S \oplus S[-1]$ is no longer connective), and obtain a pullback diagram

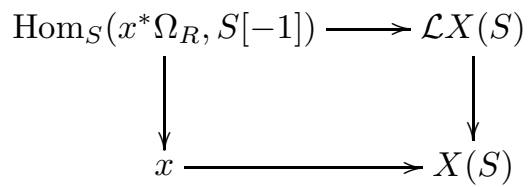

The upper left hand corner is the fiber of $\mathcal{L} X(S)$ over $x \in X(S)$. It can be identified with the fiber $x^{*} \mathbb{T}_{X}[-1]$ of the odd tangent bundle via the adjunction

$$
\operatorname{Hom}_{S}\left(x^{*} \Omega_{R}[1], S\right)=\operatorname{Hom}_{D G A_{S}^{-}}\left(x^{*} \operatorname{Sym} \bullet \Omega_{R}[1], S\right) .
$$

Thus we have an equivalence $\mathcal{L} X \simeq \mathbb{T}_{X}[-1]$ of derived schemes over $X$.

Remark 4.5. An alternate point of view on the nonconnective setting is given by [HAG2. Considering the connective $R \in D G A_{k}^{-}$as a not necessarily connective algebra, i.e., an object of $D G A_{k}$, it corresponds to an affine derived scheme in the complicial HAG theory of [HAG2]. Its cotangent complex considered in the complicial setting agrees with its cotangent complex in the connective setting since it satisfies the same universal property on a more general class of test rings.

Remark 4.6. Given a map of derived schemes $X \rightarrow X^{+}$we say that $X^{+}$is a derived thickening of $X$ if the induced map $\pi_{0}\left(\mathcal{O}_{X^{+}}\right) \rightarrow \pi_{0}\left(\mathcal{O}_{X}\right)$ is an isomorphism. We have seen above that when $X$ is a scheme, the loop space $\mathcal{L} X$ is a derived thickening of the constant loops $X$.

4.2. Equivariant sheaves and the de Rham differential. We now turn to equivariant quasicoherent sheaves on loop spaces, and relate them to the de Rham differential. We continue to assume $X$ is a quasicompact scheme with affine diagonal.

Our aim here is to describe the stable $\infty$-category of $S^{1}$-equivariant quasicoherent sheaves on the loop space $\mathcal{L} X$. Thanks to Corollary 3.15 and the canonical identification $\mathcal{L} X \simeq \mathbb{T}_{X}[-1]$ of Proposition 4.4 it suffices to describe the stable $\infty$-category of $B \mathbb{G}_{a}$-equivariant quasicoherent 
sheaves on $\mathbb{T}_{X}[-1]$. We will in fact take advantage of the evident extra symmetries and describe the stable $\infty$-category of $B \mathbb{G}_{a} \rtimes \mathbb{G}_{m}$-equivariant quasicoherent sheaves on $\mathbb{T}_{X}[-1]$.

To start, recall that we have the $\mathcal{O}_{X}$-algebra of differential forms $\Omega_{X}^{-\bullet}=\operatorname{Sym}^{\bullet}\left(\Omega_{X}[1]\right)$, and the odd tangent bundle $\mathbb{T}_{X}[-1]$ is its relative spectrum

$$
\mathbb{T}_{X}[-1]=\operatorname{Spec}_{\mathcal{O}_{X}} \Omega_{X}^{-\bullet}
$$

so that we have an equivalence

$$
\mathrm{QC}\left(\mathbb{T}_{X}[-1]\right) \simeq \Omega_{X}^{-\bullet}-\bmod .
$$

Recall that the differential graded notion or more relaxed $A_{\infty}$-notion of module are equivalent, and so there is no risk of ambiguity in our notation.

Recall as well that since $B \mathbb{G}_{a}$ is the affinization of the circle $S^{1}$, its group algebra is the formal commutative ring $H_{-*}\left(S^{1}, k\right)$. The $\mathbb{G}_{m}$-action on $B \mathbb{G}_{a}$ induces an action on $\mathbb{T}_{X}[-1]$, leading by our conventions to a Z $\mathbf{Z}$-grading $\Omega_{X}^{-\bullet}$ such that $\Omega_{X}[1]$ is of weight -1 . Thus in conclusion, we have equivalences of stable $\infty$-categories

$$
\begin{gathered}
\mathrm{QC}\left(\mathbb{T}_{X}[-1] / \mathbb{G}_{m}\right) \simeq \Omega_{X}^{-\bullet}-\bmod _{\mathbf{z}} . \\
\mathrm{QC}\left(p t /\left(B \mathbb{G}_{a} \rtimes \mathbb{G}_{m}\right)\right) \simeq H_{-*}\left(S^{1}, k\right)-\bmod _{\mathbf{z}} .
\end{gathered}
$$

We will write $\mathcal{A}_{X}$ for the sheaf of $\mathbf{Z}$-graded $k$-algebras governing quasicoherent sheaves on the quotient $\mathbb{T}_{X}[-1] /\left(B \mathbb{G}_{a} \rtimes \mathbb{G}_{m}\right)$, or in other words, $B \mathbb{G}_{a} \rtimes \mathbb{G}_{m}$-equivariant sheaves on $\mathbb{T}_{X}[-1]$. To continue in an $\mathcal{O}_{X}$-linear context, one could rather think of $\mathcal{A}_{X}$ as a $\mathbf{Z}$-graded algebra in endofunctors of $\mathrm{QC}(X)$. Informally speaking, $\mathcal{A}_{X}$ is the "semidirect product" of the algebra $\Omega_{X}^{-\bullet}$ of functions on $\mathbb{T}_{X}[-1]$ with the group algebra $H_{-*}\left(S^{1}, k\right)$ of distributions on $B \mathbb{G}_{a}$ assembled from the $B \mathbb{G}_{a}$-action on $\mathbb{T}_{X}[-1]$. It will provide us the concrete realization

$$
\mathcal{A}_{X}-\bmod \mathbf{z} \simeq \mathrm{QC}\left(\mathbb{T}_{X}[-1] /\left(B \mathbb{G}_{a} \rtimes \mathbb{G}_{m}\right)\right) .
$$

Here as elsewhere we only consider modules in the $\infty$-category of quasicoherent sheaves. So strictly speaking, an object of $\mathcal{A}_{X}-\bmod \mathbf{z}$ consists of a $\mathbf{Z}$-graded object of $\mathrm{QC}(X)$ together with module structure over $\mathcal{A}_{X}$ thought of as a $\mathbf{Z}$-graded algebra in endofunctors of $\mathrm{QC}(X)$.

Let us spell out the construction of $\mathcal{A}_{X}$ more precisely. We will describe it for $X$ affine and it will evidently form a Zariski sheaf. Consider the diagram

$$
\mathbb{T}_{X}[-1] / B \mathbb{G}_{a} \longleftrightarrow \mathbb{T}_{X}[-1] \stackrel{p}{\longrightarrow} \operatorname{Spec} k=p t
$$

First, the quotient map $q: \mathbb{T}_{X}[-1] \rightarrow \mathbb{T}_{X}[-1] / B \mathbb{G}_{a}$ provides an adjunction

$$
q^{*}: \mathrm{QC}\left(\mathbb{T}_{X}[-1] / B \mathbb{G}_{a}\right) \longleftrightarrow \mathrm{QC}\left(\mathbb{T}_{X}[-1]\right) \simeq \Omega_{X}^{-\bullet}-\bmod : q_{*}
$$

and hence a comonad $q^{*} q_{*}$ acting on $\mathrm{QC}\left(\mathbb{T}_{X}[-1]\right)$. Following the same argument as Lemma 3.10 , we see explicitly that the comonad $q^{*} q_{*}$ is given by tensoring over $k$ with the group coalgebra $\mathcal{O}\left(B \mathbb{G}_{a}\right) \simeq H^{*}\left(S^{1}, k\right)$. Furthermore, a similar invoking of the Barr-Beck theorem provides a $k$-linear equivalence

$$
\mathrm{QC}\left(\mathbb{T}_{X}[-1] / B \mathbb{G}_{a}\right) \simeq \operatorname{Comod}_{q^{*} q_{*}}\left(\mathrm{QC}\left(\mathbb{T}_{X}[-1]\right)\right) \simeq \operatorname{Comod}_{\mathcal{O}\left(B \mathbb{G}_{a}\right)}\left(\Omega_{X}^{-\bullet}-\bmod \right)
$$

Dualizing the group coalgebra $\mathcal{O}\left(B \mathbb{G}_{a}\right) \simeq H^{*}\left(S^{1}, k\right)$ gives the group algebra $\mathcal{O}\left(B \mathbb{G}_{a}\right)^{\vee} \simeq$ $H_{-*}\left(S^{1}, k\right)$, and a further $k$-linear equivalence

$$
\mathrm{QC}\left(\mathbb{T}_{X}[-1] / B \mathbb{G}_{a}\right) \simeq \operatorname{Mod}_{\mathcal{O}\left(B \mathbb{G}_{a}\right) \vee}\left(\Omega_{X}^{-\bullet}-\bmod \right) .
$$

Finally, the projection $p: \mathbb{T}_{X}[-1] \rightarrow$ Spec $k=p t$ provides an adjunction

$$
p^{*}: k-\bmod \simeq \mathrm{QC}(\operatorname{Spec} k) \longleftrightarrow \mathrm{QC}\left(\mathbb{T}_{X}[-1]\right) \simeq \Omega_{X}^{-\bullet}-\bmod : p_{*}
$$


for which $p_{*}$ is both conservative and preserves colimits since it is an affine pushforward. Hence it induces an adjunction

$$
\tilde{p}^{*}: k-\bmod \simeq \mathrm{QC}(\operatorname{Spec} k) \longleftrightarrow \operatorname{Mod}_{\mathcal{O}\left(B \mathbb{G}_{a}\right)^{\vee}}\left(\Omega_{X}^{-\bullet}-\bmod \right): \tilde{p}_{*}
$$

which satisfies the monadic form of the Barr-Beck theorem. Namely, $\tilde{p}_{*}$ is conservative and preserves colimits since it is the composition of such functors: the functor which forgets $\mathcal{O}\left(B \mathbb{G}_{a}\right)^{\vee}$-module structure and the affine pushforward $p_{*}$. Following the same argument as in Lemma 3.17, we see that the resulting monad $\tilde{p}_{*} \tilde{p}^{*}$ is given by tensoring with a $k$-algebra which we take to be $\mathcal{A}_{X}$.

All of the above constructions are $\mathbb{G}_{m}$-equivariant, and hence $\mathcal{A}_{X}$ is naturally $\mathbf{Z}$-graded. As a $\mathbf{Z}$-graded sheaf of $k$-algebras, $\mathcal{A}_{X}$ is an extension

$$
\Omega_{X}^{-\bullet} \longrightarrow \mathcal{A}_{X} \longrightarrow H_{-*}\left(S^{1}, k\right) .
$$

By construction, as a $\mathbf{Z}$-graded sheaf of $k$-modules, the extension naturally splits

$$
\mathcal{A}_{X} \simeq \Omega_{X}^{-\bullet} \otimes_{k} H_{-*}\left(S^{1}, k\right) .
$$

To see this, observe that functions $\Omega_{X}^{-\bullet}$ form a natural $\mathcal{A}_{X}$-module with canonical cyclic vector given by the unit $1 \in \mathcal{O}_{X} \subset \Omega_{X}^{-\bullet}$. Acting on the unit vector and taking the kernel provides a canonical splitting $H_{-*}\left(S^{1}, k\right) \rightarrow \mathcal{A}_{X}$.

4.2.1. The de Rham differential and formality. Now we will calculate the algebra structure on $\mathcal{A}_{X}$. By construction, the subalgebras $\Omega_{X}^{-\bullet}$ and $H_{-*}\left(S^{1}, k\right)$ appear with their usual relations. We wish to identify the relations between them in terms of the de Rham differential, which we can interpret in this general setting as the tautological vector field of degree -1 on the shifted tangent bundle $\mathbb{T}_{X}[-1]$.

Recall that the $S^{1}$-action on $\mathcal{L} X$ corresponds to the $B \mathbb{G}_{a}$-action on $\mathbb{T}_{X}[-1]$. Focusing on the action map and ignoring higher coherences, this provides a vector field of degree -1 .

Proposition 4.7. The $B \mathbb{G}_{a}$-action map on $\mathbb{T}_{X}[-1]$ is given by the canonical degree -1 vector field represented by the de Rham differential.

Proof. For any group $G$, and space $X$, consider the $G$-action on the mapping space $X^{G}=$ $\operatorname{Map}(G, X)$. The action map $a: G \times X^{G} \rightarrow X^{G}$ is evidently $G \times G$-equivariant, and hence determined by the evaluation map

$$
e v: G \times X^{G} \stackrel{a}{\longrightarrow} X^{G} \stackrel{e v_{e}}{\longrightarrow} X
$$

given by composition with evaluation at the identity $e v_{e}: X^{G} \rightarrow X$. Under adjunction, the evaluation $e v: G \times X^{G} \rightarrow X$ corresponds to the identity id : $X^{G} \rightarrow X^{G}$.

Our task is to unwind the above in the case $G=B \mathbb{G}_{a}$ and $X$ a smooth scheme. Passing to functions, we must understand the ring map

$$
\delta: \mathcal{O}_{X} \longrightarrow \Omega_{X}^{-\bullet} \otimes_{k} H^{*}\left(S^{1}, k\right) \simeq \Omega_{X}^{-\bullet}[\eta] /\left(\eta^{2}\right), \quad|\eta|=1,
$$

corresponding to the identity map $\Omega_{X}^{-\bullet} \rightarrow \Omega_{X}^{-\bullet}$. Such a map is induced by a map

$$
\delta: \mathcal{O}_{X} \longrightarrow \mathcal{O}_{X} \oplus\left(\Omega_{X}^{1}[1] \cdot \eta\right) \simeq \mathcal{O}_{X} \oplus \Omega_{X}^{1} \quad \delta(f)=\left(f, \delta_{1}(f)\right)
$$

such that $\delta_{1}$ is a derivation.

Now given any $\mathcal{O}_{X}$-module $\mathcal{M}$, we can form the linear space $M=\operatorname{Spec}_{\mathcal{O}_{X}}(\mathcal{M}[1])$ which comes equipped with a natural map $M \rightarrow \mathbb{T}_{X}[-1]$. Furthermore, given a derivation $\delta_{\mathcal{M}}: \mathcal{O}_{X} \rightarrow \mathcal{M}$, 
we obtain a corresponding $B \mathbb{G}_{a}$-action on $M$ such that the natural map $M \rightarrow \mathbb{T}_{X}[-1]$ is $B \mathbb{G}_{a}$-equivariant. Returning to action maps, this provides a factorization of the derivation

$$
\delta_{\mathcal{M}}: \mathcal{O}_{X} \stackrel{\delta_{1}}{\longrightarrow} \Omega_{X}^{1} \longrightarrow \mathcal{M}
$$

Thus $\delta_{1}$ satisfies the universal property of the de Rham differential.

In the present setting of arbitrary derived schemes over the rationals, it seems reasonable to adopt the tautological translation action of the odd line $B \mathbb{G}_{a}=\mathbb{A}^{1}[1]$ on functions on $\mathbb{T}_{X}[-1]$ as the definition of the de Rham complex. However, in the familiar and concrete setting of underived smooth schemes, there already is a standard notion of de Rham complex. Using a rigid model such as strictly commutative differential graded algebras, we can adjoin the de Rham differential $d$ to the algebra of differential forms $\Omega_{X}^{-\bullet}$ with the usual relations. In the work ToVe5, Toën and Vezzosi prove in a model categorical setting that these two notions coincide. In the present $\infty$-categorical setting, this can be seen directly by a simple weight argument which we now present.

For the rest of this section, we restrict our attention to $X$ a quasi-compact smooth underived scheme with affine diagonal over a fixed commutative rational algebra $k$.

First, we check that the classical action of the de Rham differential $d$ on the de Rham algebra $\Omega_{X}^{-\bullet}$ canonically extends to an $S^{1}$-action. We will then check that this $S^{1}$-action coincides with the rotation $S^{1}$-action on functions on the loop space.

To begin, recall that since $\Omega S^{2}$ is the free group on $S^{1}$, the action of $d$ presents $\Omega_{X}^{-}$as a sheaf of algebras over $S^{2}$ which we denote by $\mathcal{B}_{1}$. Our aim is to show that $\mathcal{B}_{1}$ extends canonically from $S^{2} \simeq \mathbf{C P}^{1}$ to a sheaf of algebras $\mathcal{B}$ over all of $B S^{1} \simeq \mathbf{C P}^{\infty}$. We will proceed by induction via the standard gluing diagram

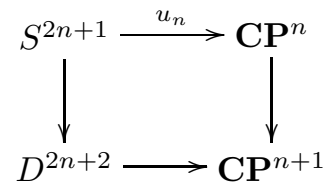

As a first step, when $n=1$, to confirm that $\mathcal{B}_{1}$ extends from $\mathbf{C P}^{1}$ to a sheaf of algebras $\mathcal{B}_{2}$ over $\mathbf{C P}^{2}$, we must trivialize the pullback $u_{1}^{*} \mathcal{B}_{1}$. The pullback $u_{1}^{*} \mathcal{B}_{1}$ is governed by its $\Omega S^{3}$-action, which since $\Omega S^{3}$ is the free group on $S^{2}$ is encoded by an endomorphism of degree -2 . Via the map $\Omega S^{3} \rightarrow \Omega S^{2}$ induced by the Hopf fibration, we see that the endomorphism is nothing more than the square $d^{2}=0$ of the de Rham differential which is identically zero. Thus we can canonically trivialize the pullback $u_{1}^{*} \mathcal{B}_{1}$ by the identically zero endomorphism $b_{-3}=0$ of degree -3 whose coboundary is the square $d^{2}=0$.

In general, suppose by induction we have constructed the sheaf of algebras $\mathcal{B}_{n}$ over $\mathbf{C P}^{n}$ via the trivializations $d$, and the zero endomorphisms $b_{-3}=\cdots=b_{-2 n+1}=0$. Then to confirm that $\mathcal{B}_{n}$ extends to a sheaf of algebras $\mathcal{B}_{n+1}$ over $\mathbf{C} \mathbf{P}^{n+1}$, we must again trivialize the pullback $u_{n}^{*} \mathcal{B}_{n}$ over $S^{2 n+1}$. Here we consider the obvious gluing diagram

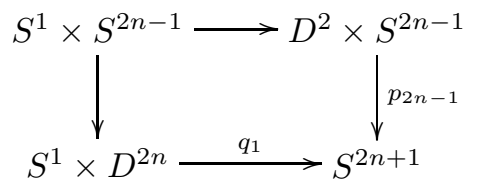

Then the pullbacks $q_{1}^{*} u_{n} \mathcal{B}_{n}$ and $p_{2 n-1}^{*} u_{n}^{*} \mathcal{B}_{n}$ are trivialized by the de Rham differential $d$ and the identically zero endomorphism $b_{2 n+1}=0$ respectively. It follows directly from standard definitions that the degree $-2 n$ endomorphism governing the pullback $u_{n}^{*} \mathcal{B}_{n}$ over $S^{2 n+1}$ is 
nothing more than the Whitehead bracket $\left[d, b_{-2 n+1}\right]$. But since $b_{-2 n+1}$ is identically zero, so is its bracket with $d$. Thus we can canonically trivialize $u_{n}^{*} \mathcal{B}_{n}$ over $S^{2 n+1}$ by taking the identically zero endomorphism $b_{-2 n-1}=0$ of degree $-2 n-1$ whose coboundary is the trivial bracket $\left[d, b_{-2 n+1}\right]=0$.

Thus to summarize, we have exhibited a canonical $S^{1}$-action on the de Rham algebra $\Omega_{X}^{-\bullet}$ whose action map is given by the standard action of the de Rham differential $d$.

Now we return to the descent algebra $\mathcal{A}_{X}$ which describes the rotation $S^{1}$-action on the algebra $\mathcal{O}_{\mathcal{L} X} \simeq \Omega_{X}^{-\bullet}$ of functions on the loop space. By Proposition 4.7, we know that the commutation relations between $\omega \in \Omega_{X}^{-\bullet}$ and the generator $\lambda \in H_{-1}\left(S^{1}, k\right)$ are given by the de Rham differential

$$
[\lambda, \omega]=d w .
$$

Our aim in what follows is to show that $\mathcal{A}_{X}$ satisfies the relations of the de Rham complex precisely. Namely, we consider $\mathcal{O}_{\mathcal{L} X} \simeq \Omega_{X}^{-\bullet}$ as an algebra with $S^{1}$-action given on the one hand by loop rotation, and on the other hand as described above by the de Rham differential. Equivalently, interpreting the $S^{1}$-action as descent data to $B S^{1}$, we consider the corresponding algebra objects over $B S^{1}$ which we denote by $\underline{\mathcal{O}}_{\mathcal{L} X}$ and $\underline{\Omega}_{X}^{-\bullet}$ respectively. Using weights, we will show that these two constructions are equivalent.

Lemma 4.8. Let $\mathcal{F}$ be a graded sheaf on $B S^{1}$ with cohomology concentrated along the diagonal weight-degree bigrading. Then any section of the restriction of $\mathcal{F}$ along the standard map $\mathbf{C P}^{1} \rightarrow$ $B S^{1}$ extends to a section of $\mathcal{F}$ over all of $B S^{1}$.

Proof. Suppose by induction we have extended to a section $\sigma_{n}$ over $\mathbf{C P}^{n}$ and seek to extend it to a section over $\mathbf{C P}^{n+1}$. Consider the standard gluing diagram

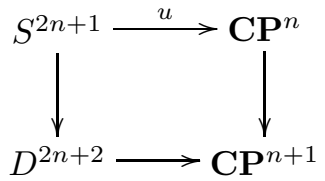

Consider the pullback $u^{*} \mathcal{F}$ and the pulled back section $u^{*} \sigma_{n}$. Then the obstruction for $\sigma_{n}$ to extend across $D^{2 n+2}$ is governed by an operator $b_{-2 n-1} \in \operatorname{Ext}^{-2 n-1}(\mathcal{F})$. In other words, $\sigma_{n}$ extends if we have $b_{-2 n-1}\left(u^{*} \sigma_{n}\right)=0$ cohomologically. Finally, observe that $b_{-2 n-1}$ is of weight grading $-n-1$, and so if $n>0$, our assumption on $\mathcal{F}$ implies that $b_{-2 n-1}$ vanishes in cohomology.

We will apply the above lemma to the sheaf of algebra morphisms

$$
\mathcal{F}=\mathcal{H o m}_{A l g / B S^{1}}\left(\underline{\mathcal{O}}_{\mathcal{L} X}, \underline{\Omega}_{X}^{-\bullet}\right) .
$$

By assumption, $X$ is smooth and underived, hence the algebra $\Omega_{X}^{-\bullet}$ is concentrated along the diagonal with cohomological degree equal to weight. The algebra equivalence $\mathcal{O}_{\mathcal{L} X} \simeq \Omega_{X}^{-\bullet}$ implies that $\mathcal{F}$ admits a canonical section over a point. The fact that $\lambda \in H_{-1}\left(S^{1}, k\right)$ acts by the de Rham differential implies that the section extends over $\mathbf{C P}^{1}$. Namely, as explained in Section 3.5.1, forgetting from $S^{1}$-actions (or objects over $B S^{1}$ ) to $\Omega S^{2}$-actions (or objects over $\mathbf{C P}^{1}$ ) corresponds to forgetting all but the action map. The lemma then implies the section extends over all of $B S^{1}$. Thus we arrive at the following conclusion.

Theorem 4.9. There is an equivalence of sheaves of $k$-algebras

$$
\mathcal{A}_{X} \simeq \Omega_{X}^{-\bullet}\langle\delta\rangle /\left(\delta^{2},[\delta, \omega]-d w\right) .
$$

In other words, $\mathcal{A}_{X}$ results from adjoining to the algebra of differential forms $\Omega_{X}^{-\bullet}$ a single element $\delta$ of degree -1 satisfying the relations of the de Rham differential. 
Remark 4.10. It is entertaining to contemplate informally the above result from a purely algebraic perspective.

A $k$-algebra with a $H_{-*}\left(S^{1}, k\right)$-action can be encoded by a degree -1 derivation $\delta_{-1}$ given by the action of $\lambda \in H_{-1}\left(S^{1}, k\right)$ together with higher coherences. The derivation $\delta_{-1}$ encodes the restriction of the algebra to $S^{2} \simeq \mathbf{C P}^{1} \subset B S^{1} \simeq \mathbf{C P}^{\infty}$. Without higher coherences, the derivation $\delta_{-1}$ would encode a $k$-algebra with a $H_{-*}\left(\Omega S^{2}, k\right)$-action, since $\Omega S^{2}$ is the free group on $S^{1}$, and hence $H_{-*}\left(\Omega S^{2}, k\right)$ is the free associative algebra on a generator of degree -1 .

The higher coherences governing a $H_{-*}\left(S^{1}, k\right)$-action impose the equations that $H_{-*}\left(S^{1}, k\right)$ is in fact commutative. So for example, the first coherence imposes the vanishing of the commutator $2 \delta_{-1}^{2}=\left[\delta_{-1}, \delta_{-1}\right]$. (Recall that $k$ is a rational algebra, so integral coefficients which arise are invertible.) This equation is realized by a degree -3 operation $\delta_{-3}$ whose coboundary is the commutator $\left[\delta_{-1}, \delta_{-1}\right]$. In fact, a $k$-algebra with a $H_{-*}\left(\Omega \mathbf{C P}^{2}, k\right)$-action is completely encoded by the operations $\delta_{-1}, \delta_{-3}$ subject to the relation that the coboundary of $\delta_{-3}$ is the commutator $\left[\delta_{-1}, \delta_{-1}\right]$.

In general, as we pass from $\mathbf{C P}^{n}$ to $\mathbf{C P}^{n+1}$, we add another operation killing previous commutators. Each of these higher operations satisfy that its $\mathbb{G}_{m}$-weight is greater than its cohomological degree. Thus for the $k$-algebra $\Omega_{X}^{-}$, which is concentrated along the diagonal of the weight-degree bigrading, all of the higher operations beyond $\delta_{-1}$ must vanish.

In differential geometry, there is a familiar relationship between quasicoherent sheaves $\mathcal{E}$ with flat connection $\nabla$ on $X$ and modules for the algebra $\mathcal{A}_{X}$. Namely, the connection $\nabla: \mathcal{E} \rightarrow$ $\mathcal{E} \otimes \Omega_{X}$ naturally extends to an $\mathcal{A}_{X}$-module structure on the pullback

$$
\pi^{*} \mathcal{E}=\mathcal{E} \otimes_{\mathcal{O}_{X}} \Omega_{X}^{-\bullet}, \quad \text { where } \pi: \mathbb{T}_{X}[-1] \rightarrow X .
$$

The action of the distinguished element $\delta \in \mathcal{A}_{X}$ is given by the formula

$$
\delta(\sigma \otimes \omega)=\nabla(\sigma) \wedge \omega+\sigma \otimes d \omega, \quad \sigma \otimes \omega \in \mathcal{E} \otimes_{\mathcal{O}_{X}} \Omega_{X}^{-\bullet} .
$$

The fact that $\nabla$ is flat leads directly to the identity $\delta^{2}=0$. To further refine this, we will consider a suitable form of Koszul duality in a later section.

4.2.2. Periodic localization. Finally, we will calculate the periodic localization of $B \mathbb{G}_{a} \rtimes \mathbb{G}_{m^{-}}$ equivariant quasicoherent sheaves on $\mathbb{T}_{X}[-1]$ (see Section 2.3 for a general discussion). We keep the hypotheses that $X$ be smooth and underived.

Since $B \mathbb{G}_{a}$ is the affinization of the circle $S^{1}$, we can identify the ring of functions on its classifying stack

$$
\mathcal{O}\left(B B \mathbb{G}_{a}\right) \simeq H^{*}\left(B S^{1}, k\right) \simeq k[u], \quad \text { with } u \text { of degree } 2 \text { and weight } 1 .
$$

Via the natural projection $\mathbb{T}_{X}[-1] /\left(B \mathbb{G}_{a} \rtimes \mathbb{G}_{m}\right) \rightarrow p t / B \mathbb{G}_{a}=B B \mathbb{G}_{a}$, we can equip the stable $\infty$-category

$$
\mathrm{QC}\left(\mathbb{T}_{X}[-1] /\left(B \mathbb{G}_{a} \rtimes \mathbb{G}_{m}\right)\right) \simeq \mathcal{A}_{X}-\bmod _{\mathbf{z}}
$$

with a module structure over $k[u]-\bmod _{\mathbf{z}}$. Recall that the periodic localization is the base change

$$
\mathcal{A}_{X}-\bmod _{p e r}=\mathcal{A}_{X}-\bmod _{\mathbf{z}} \otimes_{k[u]-\bmod \mathbf{z}} k\left[u, u^{-1}\right]-\bmod _{\mathbf{z}} \text {. }
$$

Recall as well that the periodic localization is independent of shear shifting the algebra by even degrees. To connect with familiar geometric objects, we will focus on the shear shifted algebra

$$
\mathcal{A}_{X,[-2]} \simeq \Omega_{X}^{-\bullet}\langle\delta\rangle /\left(\delta^{2},[\delta, \omega]-d w\right) .
$$

where differential forms appear in their usual nonnegative degrees, and the de Rham differential $\delta$ in degree 1. 
Let $k[t]$ be the polynomial algebra with $t$ of degree 0 and weight 1 . Then the $k[u]-\bmod _{\mathbf{Z}^{-}}$ structure on $\mathcal{A}_{X}-\bmod _{\mathbf{z}}$ translates to a $k[t]-\bmod _{\mathbf{z}}$-structure on $\mathcal{A}_{X,[-2]}$. We can calculate the periodic localization in the form

$$
\mathcal{A}_{X}-\bmod _{p e r} \simeq \mathcal{A}_{X,[-2]}-\bmod _{p e r}=\mathcal{A}_{X,[-2]}-\bmod _{\mathbf{z}} \otimes_{\mathrm{QC}\left(\mathbb{A}^{1} / \mathbb{G}_{m}\right)} \mathrm{QC}(p t),
$$

where $p t=\mathbb{G}_{m} / \mathbb{G}_{m} \subset \mathbb{A}^{1} / \mathbb{G}_{m}$ is embedded in the usual way.

We will write $\Omega_{X, d}^{\bullet}$ for the de Rham complex of $X$, thought of as a sheaf of non-formal differential graded algebras.

Theorem 4.11. There is a canonical equivalence of stable $\infty$-categories

$$
\mathrm{QC}\left(\mathbb{T}_{X}[-1]\right)_{\text {per }}^{B \mathbb{G}_{a} \rtimes \mathbb{G}_{m}} \simeq \Omega_{X, d^{-}}^{\bullet} \text { mod. }
$$

Proof. The localization functor $\mathcal{A}_{X,[-2]}-\bmod \mathbf{z} \rightarrow \mathcal{A}_{X,[-2]}-\bmod _{p e r}$ preserves colimits. Thus it suffices to identify the sheaves of $k$-algebras

$$
\Omega_{X, d}^{\bullet} \simeq \mathcal{A}_{X,[-2]} \otimes_{k[t]} k\left[t, t^{-1}\right] .
$$

This is a standard local calculation.

4.3. Holomorphic curvature. We continue with $X$ a quasi-compact smooth underived scheme with affine diagonal over $k$.

Our aim here is to describe the stable $\infty$-category of $\Omega S^{2}$-equivariant quasicoherent sheaves on $\mathcal{L} X$. Thanks to the canonical identification $\mathcal{L} X \simeq \mathbb{T}_{X}[-1]$ intertwining the natural $S^{1}$ and $B \mathbb{G}_{a}$-actions along the affinization map $S^{1} \rightarrow B \mathbb{G}_{a}$, it suffices to describe the stable $\infty$ category of $\operatorname{Aff}\left(\Omega S^{2}\right)$-equivariant quasicoherent sheaves on $\mathbb{T}_{X}[-1]$. We will keep track of the evident extra symmetries and describe the stable $\infty$-category of $\operatorname{Aff}\left(\Omega S^{2}\right) \rtimes \mathbb{G}_{m}$-equivariant quasicoherent sheaves on $\mathbb{T}_{X}[-1]$.

Following Section 3.5 and in particular Lemma 3.17, we have the sheaf of $\mathbf{Z}$-graded $k$-algebras $\tilde{\mathcal{A}}_{X}$ governing $\operatorname{Aff}\left(\Omega S^{2}\right) \rtimes \mathbb{G}_{m}$-equivariant quasicoherent sheaves on $\mathbb{T}_{X}[-1]$. Its construction follows from the adjunction given by the diagram

$$
p: \mathbb{T}_{X}[-1] / \operatorname{Aff}\left(\Omega S^{2}\right) \longrightarrow S^{2} \longrightarrow \operatorname{Spec} k=p t
$$

More specifically, over an open set, $\tilde{\mathcal{A}}_{X}$ is simply functions along the fibers of $p$. It provides us the concrete realization

$$
\mathrm{QC}\left(\mathbb{T}_{X}[-1] /\left(\operatorname{Aff}\left(\Omega S^{2}\right) \rtimes \mathbb{G}_{m}\right)\right) \simeq \tilde{\mathcal{A}}_{X}-\bmod _{\mathbf{z}} .
$$

We have the following analogue of Theorem 4.9, Consider the cohomology

$$
\mathcal{O}\left(S^{2}\right) \simeq C^{*}\left(S^{2}, k\right) \simeq H^{*}\left(S^{2}, k\right) \simeq k[u] /\left(u^{2}\right)
$$

as a graded algebra with $u$ in cohomological degree 2 and $\mathbb{G}_{m}$-weight grading 1 . Consider the $\mathbf{Z}$-graded sheaf of differential graded $k$-algebras on $X$ given by the tensor product

$$
\Omega_{X, S^{2}}^{-\bullet}=\Omega_{X}^{-\bullet} \otimes \mathcal{O}\left(S^{2}\right) \simeq \Omega_{X}^{-\bullet}[u] /\left(u^{2}\right)
$$

with differential

$$
\delta(\omega \otimes 1)=d \omega \otimes u \quad \delta(\omega \otimes u)=0
$$

Theorem 4.12. There is an equivalence of sheaves of $k$-algebras

$$
\tilde{\mathcal{A}}_{X} \simeq \Omega_{X, S^{2}}^{-\bullet}
$$

Proof. The assertion follows from the calculation of the $S^{1}$-action map in Proposition 4.7.

We will interpret the theorem in terms of sheaves with not necessarily flat connection in the section immediately following. 


\section{Koszul DuAl Description}

This section contains reformulations of results from the previous section in a Koszul dual language. In the previous section, we identified equivariant sheaves on the loop space of a derived scheme $X$ with equivariant sheaves on the odd tangent bundle $\mathbb{T}_{X}[-1]$ or modules over the de Rham algebra $\mathcal{A}_{X}$. In this section, we will give a Koszul dual description in terms of modules over the Rees algebra of differential operators, or in other words, in terms of sheaves on a noncommutative deformation of the cotangent bundle $\mathbb{T}_{X}^{*}$, inspired by $\mathrm{Ka}$ ] and BD] as well as GKM]. As a result, we can describe equivariant sheaves on loop spaces in terms of $\mathcal{D}$-modules. The discussion will rely on the yoga of gradings, weights and periodic localization described in Section 2.3. Although we provide some details, none of the arguments concerning Koszul duality are really new.

We continue to assume throughout this section that $X$ is a quasi-compact smooth underived scheme with affine diagonal. In particular, we can appeal to the well developed theory of differential operators and Koszul duality. (Presumably the results hold in greater generality once differential operators are defined appropriately.)

5.1. Graded Koszul duality. Let $\mathcal{D}_{X}$ be the sheaf of differential operators on $X$, and for $i \geq 0$, let $\mathcal{D}_{X}^{\leq i} \subset \mathcal{D}_{X}$ be the differential operators of order at most $i$. In particular, $\mathcal{D}_{X}^{\leq 0}$ is the commutative ring of functions $\mathcal{O}_{X}$, and $\mathcal{D}_{X}^{\leq 1}$ is the usual Picard Lie algebroid of vector fields $\mathbb{T}_{X}$ extended by functions $\mathcal{O}_{X}$.

As usual, by a $\mathcal{D}_{X}$-module, we will mean a quasicoherent $\mathcal{O}_{X}$-module with a compatible action of $\mathcal{D}_{X}$.

Next, recall the standard graded Rees algebra associated to $\mathcal{D}_{X}$ with its filtration by order of operator. Consider the $\mathbb{G}_{m}$-action on $\mathbb{A}^{1}=$ Spec $k[t]$ so that $t$ has weight 1 . The Rees algebra $\mathcal{R}_{X}$ is the sheaf of $\mathbf{Z}$-graded $k$-algebras

$$
\mathcal{R}_{X}=\bigoplus_{i \geq 0} t^{i} \mathcal{D}_{X}^{\leq i} \subset \mathcal{D}_{X} \otimes_{k} k[t] .
$$

Note that $\mathcal{R}_{X}$ contains $k[t]=\mathcal{O}\left(\mathbb{A}^{1}\right)$ as a central subalgebra, and the $\mathbf{Z}$-grading on $\mathcal{R}_{X}$ reflects the fact that $\mathcal{R}_{X}$ descends from $X \times \mathbb{A}^{1}$ to the quotient $X \times\left(\mathbb{A}^{1} / \mathbb{G}_{m}\right)$.

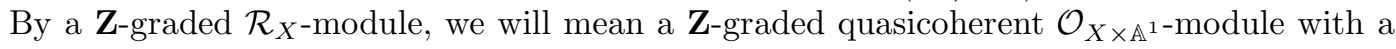
compatible action of $\mathcal{R}_{X}$.

The fiber of $\mathcal{R}_{X}$ at the origin is the commutative associated graded

$$
\mathcal{R}_{X} \otimes_{k[t]} k[t] /(t) \simeq \operatorname{gr} \mathcal{D}_{X} \simeq \mathcal{O}_{\mathbb{T}_{X}^{*}} \simeq \operatorname{Sym}^{\bullet}\left(\mathbb{T}_{X}\right)
$$

The restriction of $\mathcal{R}_{X}$ to the generic orbit $p t=\mathbb{G}_{m} / \mathbb{G}_{m} \subset \mathbb{A}^{1} / \mathbb{G}_{m}$ is the original algebra $\mathcal{D}_{X}$, or equivalently, there is a canonical isomorphism of $\mathbf{Z}$-graded algebras

$$
\mathcal{R}_{X} \otimes_{k[t]} k\left[t, t^{-1}\right] \simeq \mathcal{D}_{X} \otimes_{k} k\left[t, t^{-1}\right]
$$

Thus the periodic localization of $\mathcal{R}_{X}$-modules are simply $\mathcal{D}_{X}$-modules

$$
\mathcal{R}_{X}-\bmod _{\text {per }}=\mathcal{R}_{X}-\bmod _{\mathbf{z}} \otimes_{k[t]-\bmod \mathbf{z}} k\left[t, t^{-1}\right]-\bmod _{\mathbf{z}} \simeq \mathcal{D}_{X}-\bmod
$$

For usual technical reasons, we will work with a completed version of the Rees algebra. Consider the $\mathbb{G}_{m}$-action on the ind-scheme $\widehat{\mathbb{A}}^{1}=\operatorname{Spf} k[[t]]$ so that $t$ has weight 1 . We define the completed Rees algebra $\widehat{\mathcal{R}}_{X}$ to be the sheaf of completed $\mathbf{Z}$-graded $k$-algebras

$$
\widehat{\mathcal{R}}_{X}=\lim _{j \rightarrow \infty} \mathcal{R}_{X} / \oplus_{i \geq j} t^{i} \mathcal{D}_{X}^{\leq i} \subset \mathcal{D}_{X} \otimes_{k} k[[t]]
$$


Recall that the $\mathbb{G}_{m}$-action on $\mathbb{A}^{1}$ places $t$ in weight 1 , hence $\widehat{\mathcal{R}}_{X}$ is the completion of $\mathcal{R}_{X}$ with respect to the positive weight direction. The fiber of $\widehat{\mathcal{R}}_{X}$ at the origin is the ring of functions on the completion of $\mathbb{T}_{X}^{*}$ along the zero section

$$
\widehat{\mathcal{R}}_{X} \widehat{\otimes}_{k[[t]]} k[[t]] /(t) \simeq \lim _{j \rightarrow \infty} \operatorname{Sym}^{\bullet}\left(\mathbb{T}_{X}\right) / \oplus_{i \geq j} \operatorname{Sym}^{i}\left(\mathbb{T}_{X}\right) \simeq \mathcal{O}_{\widehat{\mathbb{T}}_{X}^{*}}
$$

Now we have the following noncommutative form of Koszul duality (see also [Ka], $\mathrm{BD}$ ).

Theorem 5.1. There is a canonical equivalence of stable $\infty$-categories

$$
\mathcal{A}_{X}-\bmod _{\mathbf{z}} \simeq \widehat{\mathcal{R}}_{X}-\operatorname{cmod}_{\mathbf{z}}
$$

Proof. It is more natural to give an equivalence

$$
\mathcal{A}_{X,[-2]}-\bmod _{\mathbf{z}} \simeq \widehat{\mathcal{R}}_{X}-\operatorname{cmod}_{\mathbf{z}}
$$

and then use Proposition 2.6 to arrive at the assertion.

We will give a proof via the $\infty$-categorical Barr-Beck theorem of [L2, 6.2.2]. Consider the adjoint functors

$$
\begin{gathered}
\ell: \mathcal{O}_{X}-\bmod \mathbf{z} \leftrightarrows \widehat{\mathcal{R}}_{X}-\operatorname{cmod}_{\mathbf{Z}}: r \\
\ell(\mathcal{F})=\mathcal{F} \quad r(\mathcal{M})=\operatorname{Hom}_{\widehat{\mathcal{R}}_{X}}\left(\mathcal{O}_{X}, \mathcal{M}\right) .
\end{gathered}
$$

where $\ell$ is simply the pushforward along the natural augmentation $\widehat{\mathcal{R}}_{X} \rightarrow \mathcal{O}_{X}$. Note that $r$ also preserves colimits since $\mathcal{O}_{X}$ is dualizable and so compact (it can be realized by a finite Koszul resolution). Furthermore, $r$ is conservative by Nakayama's lemma (as in the proof of Theorem 6.9).

Let $T=r \circ \ell$ be the resulting monad. By the Barr-Beck theorem, we have an equivalence

$$
\widehat{\mathcal{R}}_{X}-\operatorname{cmod}_{\mathbf{z}} \simeq \operatorname{Mod}_{T}\left(\mathcal{O}_{X}-\bmod _{\mathbf{z}}\right)
$$

It is a standard calculation (see for example [Ka], $\mathrm{BD}]$ ) that $T$ is given by the algebra $\mathcal{A}_{X,[-2]} \simeq$ $\operatorname{Hom}_{\widehat{\mathcal{R}}_{X}}\left(\mathcal{O}_{X}, \mathcal{O}_{X}\right)$.

A shortcoming of the equivalence of Theorem 5.1 is that involves the completed Rees algebra rather than the ordinary version. To remedy this, we can restrict to stable $\infty$-categories of suitably finite modules. Let us write $\operatorname{Perf}_{X}(\mathcal{L} X)$ for the small, stable $\infty$-subcategory of $\mathrm{QC}(\mathcal{L} X)$ of quasicoherent sheaves whose pushforward along the canonical map $\mathcal{L} X \rightarrow X$ are perfect. Note that the structure sheaf $\mathcal{O}_{\mathcal{L X}} \simeq \operatorname{Sym}_{\mathcal{O}_{X}}\left(\Omega_{X}[1]\right)$ is perfect over $\mathcal{O}_{X}$, and the $\mathcal{O}_{\mathcal{L} X}$-augmentation module $\mathcal{O}_{X}$ is obviously perfect over $\mathcal{O}_{X}$ (though not over $\mathcal{O}_{\mathcal{L} X}$ ). We write $\mathcal{R}_{X}$-perf for the stable $\infty$-category of quasicoherent sheaves on $X$ equipped with a compatible structure of perfect $\mathcal{R}_{X}$-module.

Corollary 5.2. There is a canonical equivalence of small, stable $\infty$-categories

$$
\operatorname{Perf}_{X}(\mathcal{L} X)^{B \mathbb{G}_{a} \rtimes \mathbb{G}_{m}} \simeq \mathcal{R}_{X}-\operatorname{perf}_{\mathbf{Z}}
$$

Finally, we observe that the functors realizing the equivalence of Theorem 5.1 are naturally linear over $\mathcal{O}\left(B B \mathbb{G}_{a}\right) \simeq H^{*}\left(B S^{1}, k\right)$, hence the equivalence is linear and we can pass to its periodic localization. In particular, if we take the image of the above equivalence under periodic localization, we obtain a canonical equivalence of perfect modules.

Corollary 5.3. There is a canonical equivalence of small, stable $\infty$-categories

$$
\operatorname{Perf}_{X}(\mathcal{L} X)_{\text {per }}^{B \mathbb{G}_{a} \rtimes \mathbb{G}_{m}} \simeq \mathcal{D}_{X} \text {-perf. }
$$


5.2. Twisted $\mathcal{D}$-modules. Our aim here is to interpret $\Omega S^{2}$-equivariant quasicoherent sheaves on $\mathcal{L} X$ in terms of Rees modules. The arguments follow the same pattern as in the previous section and we leave it to the reader to repeat them as necessary,

Thanks to the canonical identification $\mathcal{L} X \simeq \mathbb{T}_{X}[-1]$ intertwining the natural $S^{1}$ and $B \mathbb{G}_{a^{-}}$ actions along the affinization map $S^{1} \rightarrow B \mathbb{G}_{a}$, it suffices to describe $\operatorname{Aff}\left(\Omega S^{2}\right)$-equivariant quasicoherent sheaves on $\mathbb{T}_{X}[-1]$. We will in fact work with the evident extra symmetries and describe $\operatorname{Aff}\left(\Omega S^{2}\right) \rtimes \mathbb{G}_{m}$-equivariant quasicoherent sheaves.

Recall that for $i \geq 0$, we write $\mathcal{D}_{X}^{\leq i} \subset \mathcal{D}_{X}$ for the differential operators of order at most $i$. In particular, $\mathcal{D}_{\bar{X}}^{\leq 0}$ is the commutative ring of functions $\mathcal{O}_{X}$, and $\mathcal{D}_{\bar{X}}^{\leq 1}$ is the usual Picard Lie algebra of vector fields $\mathbb{T}_{X}$ extended by functions $\mathcal{O}_{X}$. By convention, for $i<0$, we set $\mathcal{D}_{\bar{X}}^{\leq i}=\{0\}$.

Consider the $\mathbb{G}_{m}$-action on $\mathbb{A}^{1}=\operatorname{Spec} k[t]$ so that $t$ has weight 1 . Following $[\mathrm{BB}$, we define the subprincipal Rees algebra to be the $\mathbf{Z}$-graded algebra

$$
\mathcal{R}_{X}^{s p}=\mathcal{R}_{X} \otimes_{k[t]} k[t] /\left(t^{2}\right) \simeq \bigoplus_{i \geq 0} t^{i}\left(\mathcal{D}_{\bar{X}}^{\leq i} / \mathcal{D}_{\bar{X}}^{\leq i-2}\right) .
$$

The shear shifted algebra $\mathcal{R}_{X,[-2]}^{s p}$ admits the following topological interpretation. If we think of $\mathcal{R}_{X,[-2]}$ as an algebra over $\mathcal{O}\left(B S^{1}\right)=k[u]$, with $u$ of degree 2 and weight 1 , then $\mathcal{R}_{X,[-2]}^{s p}$ is the base change to $\mathcal{O}\left(S^{2}\right)=k[u] /\left(u^{2}\right)$.

Let $\widehat{\mathcal{R}}_{X}^{s p}$ be the completion of $\mathcal{R}_{X}^{s p}$ with respect to the positive weight direction

$$
\widehat{\mathcal{R}}_{X}^{s p}=\lim _{j \rightarrow \infty} \mathcal{R}_{X}^{s p} / \bigoplus_{i \geq j} t^{i}\left(\mathcal{D}_{X}^{\leq i} / \mathcal{D}_{X}^{\leq i-2}\right)
$$

Now Theorem 4.12 and an application of Koszul duality to the natural $\Omega_{X, S^{2}}^{-\bullet}$-augmentation module $\mathcal{O}_{X} \otimes \mathcal{O}\left(S^{2}\right)$ provides the following.

Theorem 5.4. There is a canonical equivalence of stable $\infty$-categories

$$
\mathrm{QC}(\mathcal{L} X)^{\mathrm{Aff}\left(\Omega S^{2}\right) \rtimes \mathbb{G}_{m}} \simeq \widehat{\mathcal{R}}_{X}^{s p}-\operatorname{cmod}_{\mathbf{z}}
$$

Alternatively, we can consider the natural $\Omega_{X, S^{2}}^{-\bullet}$-augmentation module $\Omega_{X}^{-\bullet}$. Let $\Omega_{X}^{-\bullet}\langle d\rangle$ be the $\mathbf{Z}$-graded sheaf of differential graded $k$-algebras on $X$ obtained from the de Rham algebra by adjoining an element $d$ of degree -1 with $[d, \omega]=d \omega$, for $\omega \in \Omega_{X}^{-\bullet}$, but with no equation on powers of $d$. We write $\Omega_{X}^{-\bullet}\langle d\rangle$-cmod for the stable $\infty$-category of quasicoherent sheaves on $X$ equipped with a compatible structure of complete differential graded $\Omega_{X}^{-\bullet}\langle d\rangle$-module.

Corollary 5.5. There is a canonical equivalence of stable $\infty$-categories

$$
\mathrm{QC}(\mathcal{L} X)^{\mathrm{Aff}\left(\Omega S^{2}\right) \rtimes \mathbb{G}_{m}} \simeq \Omega_{X}^{-\bullet}\langle d\rangle-\operatorname{cmod}_{\mathbf{z}}
$$

Recall the discussed relationship between quasicoherent sheaves $\mathcal{E}$ with flat connection $\nabla$ on $X$ and modules for the algebra $\Omega_{X}^{-\bullet}[d]$. It generalizes to a similar relationship between quasicoherent sheaves $\mathcal{E}$ with not necessarily flat connection $\nabla$ and modules for the algebra $\Omega_{X}^{-\bullet}\langle d\rangle$. Namely, the connection $\nabla: \mathcal{E} \rightarrow \mathcal{E} \otimes \Omega_{X}$ naturally extends to an $\Omega_{X}^{-\bullet}\langle d\rangle$-module structure on the pullback

$$
\pi^{*} \mathcal{E}=\mathcal{E} \otimes_{\mathcal{O}_{X}} \Omega_{X}^{-\bullet}, \quad \text { where } \pi: \mathbb{T}_{X}[-1] \rightarrow X .
$$

The action of the distinguished element $d \in \Omega_{X}^{-\bullet}\langle d\rangle$ is given by the formula

$$
d(\sigma \otimes \omega)=\nabla(\sigma) \wedge \omega+\sigma \otimes d \omega, \quad \sigma \otimes \omega \in \mathcal{E} \otimes_{\mathcal{O}_{X}} \Omega_{X}^{-\bullet} .
$$

Here we do not require that $\nabla$ is flat nor correspondingly that the square of $d$ vanishes. The square of $d$ is given by the curvature $R=\nabla^{2}: \mathcal{E} \rightarrow \mathcal{E} \otimes \Omega_{X}^{\wedge 2}$. 
5.2.1. Central curvature. We specialize the above discussion to fixed central curvature.

We will write $\mathcal{K}_{X}$ for the tensor product of differential graded $k$-algebras

$$
\mathcal{K}_{X}=\Omega_{X}^{-\bullet} \otimes H_{-*}\left(\Omega S^{3}, k\right) \simeq \Omega_{X}^{-\bullet}[\kappa]
$$

where $\kappa$ has cohomological degree -2 and weight grading -2 . Since $\operatorname{Aff}\left(\Omega S^{3}\right)$ lies in the kernel of the morphism $\operatorname{Aff}\left(\Omega S^{2}\right) \rightarrow \operatorname{Aff}\left(S^{1}\right)=B \mathbb{G}_{a}$, following the same arguments as above, we can obtain a description of $\operatorname{Aff}\left(\Omega S^{3}\right) \rtimes \mathbb{G}_{m}$-equivariant sheaves on $\mathbb{T}_{X}[-1]$ in terms of complete differential graded modules

$$
\mathrm{QC}\left(\mathbb{T}_{X}[-1] /\left(\operatorname{Aff}\left(\Omega S^{3}\right) \rtimes \mathbb{G}_{m}\right) \simeq \mathcal{K}_{X}-\operatorname{cmod}_{\mathbf{Z}} .\right.
$$

Observe that the map $\Omega S^{3} \rightarrow \Omega S^{2}$ induces a map $\mathcal{K}_{X} \rightarrow \Omega_{X}^{-\bullet}\langle d\rangle$ such that $\kappa \mapsto d^{2}$. Thanks to Theorems 4.9 and 4.12 and the previous Koszul duality reformulations, we see that a $B \mathbb{G}_{a} \rtimes \mathbb{G}_{m^{-}}$ equivariant sheaf on $\mathbb{T}_{X}[-1]$ is nothing more than an $\operatorname{Aff}\left(\Omega S^{2}\right) \rtimes \mathbb{G}_{m}$-equivariant sheaf on which $\operatorname{Aff}\left(\Omega S^{3}\right)$ acts trivially. More precisely, if we write $k_{0}$ for the natural augmentation $k[\kappa]$-module on which $\kappa$ acts trivially, then we can understand $\Omega_{X}^{-\bullet}[d]$-modules via the naive identification of differential graded $k$-algebras

$$
\Omega_{X}^{-\bullet}[d] \simeq \Omega_{X}^{-\bullet}\langle d\rangle \otimes_{k[\kappa]} k_{0}
$$

In other words, a differential graded $\Omega_{X}^{-\bullet}[d]$-module is a differential graded $\Omega_{X}^{-\bullet}\langle d\rangle$-module whose restriction to a differential graded $\mathcal{K}_{X}$-module factors through the natural projection

$$
\mathcal{K}_{X}=\Omega_{X}^{-\bullet} \otimes H_{-*}\left(\Omega S^{3}, k\right) \longrightarrow \Omega_{X}^{-\bullet} .
$$

In general, given an $\Omega_{X}^{-\bullet}\langle d\rangle$-module, we could ask that its restriction to a $\mathcal{K}_{X}$-module factors through an alternative algebra projection $\mathcal{K}_{X} \rightarrow \Omega_{X}^{-\bullet}$. Given a morphism

$$
\chi: H_{-*}\left(\Omega S^{3}, k\right) \longrightarrow \Gamma\left(X, \Omega_{X}^{-\bullet}\right),
$$

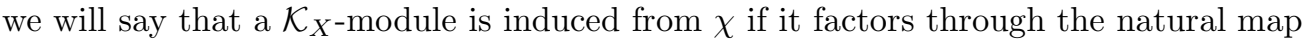

$$
\text { id } \otimes \chi: \mathcal{K}_{X}=\Omega_{X}^{-\bullet} \otimes H_{-*}\left(\Omega S^{3}, k\right) \longrightarrow \Omega_{X}^{-\bullet}
$$

induced by restriction and multiplication. Observe that $\chi$ is completely determined by the function $\chi(\kappa) \in \Omega_{X}^{-2}$.

Given an $\Omega_{X}^{-\bullet}\langle d\rangle$-module, we will say that it has curvature $\chi$ if its restriction to a $\mathcal{K}_{X}$-module is induced from $\chi$. Such modules are nothing more than differential graded modules for the sheaf of differential graded $k$-algebras

$$
\mathcal{A}_{X}^{\chi} \simeq \Omega_{X}^{-\bullet}\langle d\rangle \otimes_{k[\kappa]} k_{\chi}
$$

where $k_{\chi}$ is the $H_{-*}\left(\Omega S^{3}, k\right)$-module given by $\chi$. Of course, when $\chi$ is trivial, we recover the original differential graded algebra $\mathcal{A}_{X}^{0}=\mathcal{A}_{X} \simeq \Omega_{X}^{-\bullet}[d]$.

Observe that for an arbitrary $\chi$ to arise as such a restriction, its value $\chi(\kappa)$ must be a de Rham closed element of $\Omega_{X}^{-2}$ since $\chi(\kappa)=\tilde{\delta}^{2}$ commutes with $\tilde{\delta}$. Furthermore, two such morphisms $\chi_{1}$ and $\chi_{2}$ lead to equivalent algebras $\mathcal{A}_{X}^{\chi_{1}} \simeq \mathcal{A}_{X}^{\chi_{2}}$ whenever the difference $\chi_{2}(\kappa)-\chi_{1}(\kappa)$ is the de Rham boundary of some $\alpha$. In this case, we can define an equivalence $\varphi_{\alpha}: \mathcal{A}_{X}^{\chi_{1}} \rightarrow \mathcal{A}_{X}^{\chi_{2}}$ to be the identity on $\Omega_{X}^{-\bullet}$ extended by $\varphi_{\alpha}(\tilde{\delta})=\tilde{\delta}+\alpha$. Thus possible central characters are classified by the holomorphic part of the second de Rham cohomology of $X$.

Now consider a quasicoherent sheaf $\mathcal{E}$ with connection $\nabla$ on $X$. Assume that the curvature $R=\nabla^{2}: \mathcal{E} \rightarrow \mathcal{E} \otimes \Omega_{X}^{\wedge}$ is central in the sense that it factors as a tensor product $R=\operatorname{id}_{\mathcal{E}} \otimes \omega$, for some $\omega \in \Omega_{X}^{\wedge 2}$. Then the $\Omega_{X}^{-\bullet}\langle d\rangle$-module structure on the pullback

$$
\pi^{*} \mathcal{E}=\mathcal{E} \otimes_{\mathcal{O}_{X}} \Omega_{X}^{-\bullet}, \quad \text { where } \pi: \mathbb{T}_{X}[-1] \rightarrow X,
$$


descends to an $\mathcal{A}_{X}^{\chi}$-module structure with $\chi$ defined by $\chi(\kappa)=\omega$.

To summarize, sheaves with connection on $X$ naturally give $\operatorname{Aff}\left(\Omega S^{2}\right)$-equivariant sheaves on $\mathbb{T}_{X}[-1]$. When the connection is flat, they descend to $B \mathbb{G}_{a}$-equivariant sheaves. When the connection is central, they can be understood as $\operatorname{Aff}\left(\Omega S^{2}\right)$-equivariant sheaves on which $\operatorname{Aff}\left(\Omega S^{3}\right)$ acts by a character.

\section{LOOPS IN GEOMETRIC STACKS}

In this section, we turn to loops in derived stacks and generalize the results of previous sections. We continue to work over a fixed $\mathbf{Q}$-algebra $k$. We will only consider geometric derived stacks, by which we connote Artin derived stacks with affine diagonal HAG2. Such stacks have two related advantages: first, they admit smooth affine covers by affine derived schemes (in other words, they can be realized as the colimits of simplicial affine derived schemes with smooth face maps), enabling us to reduce questions to the affine case; and second, they have well behaved infinitesimal theory as captured by their cotangent complexes. We will show that all of our results on loop spaces of derived schemes extend to formal loop spaces of arbitrary geometric stacks.

6.1. Loops, unipotent loops, and formal loops. For $X$ a derived scheme, we found equivalences

$$
\mathbb{T}_{X}[-1] \stackrel{\sim}{\longrightarrow} \operatorname{Map}\left(B \mathbb{G}_{a}, X\right) \stackrel{\sim}{\longrightarrow} \operatorname{Map}\left(S^{1}, X\right)=\mathcal{L} X
$$

identifying the zero section $X \rightarrow \mathbb{T}_{X}[-1]$ with the constant loops $X \rightarrow \mathcal{L} X$. Furthermore, $\mathbb{T}_{X}[-1]$ is complete along its zero section, and equivalently $\mathcal{L} X$ is complete along constant loops.

For a general derived stack, these identifications break down. We therefore make the following definition to distinguish between the possible constructions.

Definition 6.1. The unipotent loop space of a derived stack $X$ is the derived mapping stack

$$
\mathcal{L}^{u} X=\operatorname{Map}_{D S t}\left(B \mathbb{G}_{a}, X\right) .
$$

The formal loop space of $X$ is the formal completion of $\mathcal{L} X$ along the constant loops

$$
\widehat{\mathcal{L}} X=\widehat{\mathcal{L} X}_{X} .
$$

The formal odd tangent bundle of $X$ is the formal completion of $\mathbb{T}_{X}[-1]$ along its zero section

$$
\left.\widehat{\mathbb{T}}_{X}[-1]=\widehat{\mathbb{T}_{X}[-1}\right]_{X}=\operatorname{Spf}_{\mathcal{O}_{X}} \lim _{n \rightarrow \infty}\left(\operatorname{Sym}^{\bullet}\left(\Omega_{X}[1]\right) / \operatorname{Sym}^{>n}\left(\Omega_{X}[1]\right)\right) .
$$

Remark 6.2. By the formal spectrum $\operatorname{Spf}$ above we connote the corresponding ind-derived scheme over $X$, i.e., the direct limit functor.

Recall that if we realize $S^{1}$ by two 0 -simplices connected by two 1 -simplices, we obtain the identification

$$
\mathcal{L} X \simeq X \times_{X \times X} X .
$$

This guarantees that for $X$ an Artin derived stack, the loop space $\mathcal{L} X$ is again Artin. Moreover, when $X$ has affine diagonal, the natural projection $\mathcal{L} X \rightarrow X$ is affine since it is a basechange of the diagonal of $X$. 
6.1.1. Relation to inertia stacks. It is illuminating to note that the derived loop space of a stack is an infinitesimal thickening of the classical inertia stack. First recall that to any derived stack $Z$, there is an underived stack $t(Z)$ obtained by restricting the functor of points of $Z$ to discrete rings. One refers to $t(Z)$ as the truncation of $Z$.

Definition 6.3. For an underived stack $\underline{X}$, the inertia stack $I \underline{X}$ is the underived loop space

$$
I \underline{X}=\operatorname{Map}^{\prime}\left(S^{1}, \underline{X}\right) \simeq \underline{X} \times^{\prime} \underline{X} \times \underline{X} \underline{X} .
$$

Here both the mapping functor Map' and fiber product $x^{\prime}$ are calculated in the $\infty$-category of stacks over discrete $k$-algebras.

Thus for $S$ a discrete $k$-algebra, the $S$-points $I \underline{X}(S)$ of the inertia stack are pairs consisting of an $S$-point $x \in \underline{X}(S)$, and an automorphism $\gamma \in \operatorname{Aut}_{\underline{X}(S)}(x)$ of the point (or in other words, a loop based at $x$ ). Composition of loops makes $\underline{I} X$ a group stack over $\underline{X}$, and when $\underline{X}$ has affine diagonal, $I \underline{X}$ is an affine group scheme over $\underline{X}$. Likewise one can consider the unipotent inertia stack $I^{u} \underline{X}$. which classifies algebraic one-parameter subgroups of the inertia stack.

We observe that the truncation $t(\mathcal{L} X)$ of the loop space $\mathcal{L} X$ can be described concretely as the inertia stack $I \underline{X}$ of the truncation $\underline{X}$ of $X$. This is an immediate consequence of the fact that truncation of derived stacks preserves limits (it is right adjoint to inclusion of stacks).

above

6.2. Descent for differential forms. In this section, we explain a descent pattern for differential forms, or in other words, functions on odd tangent bundles.

We begin with some notation and standard constructions. First, for a geometric stack $Z$, the quasicoherent sheaf $\Omega_{Z}^{k} \in \mathrm{QC}(Z)$ will denote the $k$ th symmetric power of the shifted cotangent complex $\Omega_{Z}^{1}=\Omega_{Z}[1]$.

For $f: Z \rightarrow W$ a representable map of derived stacks, and any integer $k \geq 0$, we define the quasi-coherent sheaf $\Omega_{Z / W}^{k} \in \mathrm{QC}(Z)$ of relative $k$-forms to be the cone

$$
f^{*} \Omega_{W}^{k} \stackrel{f^{*}}{\longrightarrow} \Omega_{Z}^{k} \longrightarrow \operatorname{Cone}\left(f^{*}\right)=\Omega_{Z / W}^{k} .
$$

Of course, when $k=0$, we have $\Omega_{Z / W}^{0} \simeq 0$, and when $k=1$, we recover the usual sheaf of relative 1-forms. Informally speaking, we can think of $\Omega_{Z / W}^{k}$ as the $k$-forms on $Z$ which vanish when contracted with vector fields along the fibers of $f$.

For a diagram of representable maps

$$
Z \stackrel{f}{\longrightarrow} W \longrightarrow V
$$

and for any integer $k \geq 0$, we have a natural triangle

$$
f^{*} \Omega_{W / V}^{k} \longrightarrow \Omega_{Z / V}^{k} \longrightarrow \Omega_{Z / W}^{k}
$$

Moreover, when $f$ is smooth, then locally the sequence admits a splitting

$$
f^{*} \Omega_{W / V}^{k} \stackrel{s}{\stackrel{s}{f^{*}}} \Omega_{Z / V}^{k} \longrightarrow \Omega_{Z / W}^{k}, \quad s \circ f^{*} \simeq \mathrm{id} .
$$

(This is a consequence of the definition of smoothness as local projectivity of the relative tangent complex, see [HAG2].)

Now we will address the descent pattern for differential forms. Fix a geometric stack $X$. By a cover $u: U \rightarrow X$, we will always mean a faithfully flat map, i.e., a cover in the flat topology on 
derived rings as defined in [L3, 5]. By [L3, Theorem 6.1], quasicoherent sheaves satisfy descent for flat covers, so that if $u: U \rightarrow X$ is a cover, then pullback provides an equivalence

$$
\mathrm{QC}(X) \stackrel{\sim}{\longrightarrow} \lim \mathrm{QC}\left(U_{\bullet}\right)
$$

where $U_{\bullet} \rightarrow X$ is the corresponding augmented Cech simplicial derived stack. We will also need the smooth topology on derived rings, which is the refinement of the flat topology in which covering maps are required to be smooth (this forms a Grothendieck topology thanks to the criterion of [L3, Proposition 5.1]). By a smooth affine cover, we mean a cover $u: U \rightarrow X$ for which $U$ is affine and $u$ is smooth. Note that in this case, $U$ • will be a simplicial affine derived scheme with smooth face maps.

Some notation: let $u_{\bullet}: U_{\bullet} \rightarrow X$ be the unique augmentation map, so that in particular $u_{0}: U_{0}=U \rightarrow X$ is the original cover $u$.

Proposition 6.4. For a geometric stack $X$ and smooth maps $U \rightarrow X$, the assignment of the complete graded algebra of differential forms

$$
U \longmapsto \widehat{\operatorname{Sym}} \bullet \Omega_{X}[1]=\lim _{n \rightarrow \infty}\left(\operatorname{Sym}^{\bullet}\left(\Omega_{X}[1]\right) / \operatorname{Sym}^{>n}\left(\Omega_{X}[1]\right)\right)
$$

forms a graded sheaf on the smooth site of $X$. In particular, for all $k \geq 0$, the assignment of shifted $k$-forms

$$
U \longmapsto \Omega_{U}^{k}=\operatorname{Sym}^{k}\left(\Omega_{U}[1]\right)
$$

forms a sheaf on the smooth site of $X$.

Proof. Fix a smooth affine cover $u: U \rightarrow X$ with corresponding augmented Cech simplicial affine derived scheme $U_{\bullet} \rightarrow X$. It suffices to show that pullback induces an equivalence

$$
\widehat{\operatorname{Sym}} \Omega_{X}[1] \stackrel{\sim}{\longrightarrow} \operatorname{Tot}\left[u_{\bullet *}\left(\widehat{\operatorname{Sym}} \Omega_{U}[1]\right)\right]
$$

where we write Tot for the limit of a cosimplicial object.

Let us first fix $k \geq 0$ and consider the assertion for $k$-forms alone. In other words, we seek to confirm that pullback induces an equivalence

$$
\Omega_{X}^{k} \stackrel{\sim}{\longrightarrow} \operatorname{Tot}\left[u_{\bullet *}\left(\Omega_{U_{\bullet}}^{k}\right)\right]
$$

Note that for $k=0$, this is simply descent for functions.

Since cones can be interpreted as limits, and limits always commute with limits, it suffices to establish the equivalence

$$
0 \simeq \Omega_{X / X}^{k}[1] \stackrel{\sim}{\longrightarrow} \operatorname{Tot}\left[u_{\bullet *}\left(\Omega_{U_{\bullet} / X}^{k}[1]\right)\right], \quad \text { for any } k>0 .
$$

Let us pull back the augmented cosimplicial object $0 \rightarrow u_{\bullet *}\left(\Omega_{U_{\bullet} / X}^{k}[1]\right)$ along the initial augmentation map $u_{0}: U_{0} \rightarrow X$. Using base change and the splitting of cotangent sequences for smooth maps, it is a diagram chase to confirm that the pullback $0 \rightarrow u_{0}^{*} u_{\bullet *}\left(\Omega_{U_{\bullet} / X}^{k}[1]\right)$ is a split cosimplicial object, and hence a limit diagram. Since $u_{0}$ is a smooth, in particular flat, cover (and hence $u_{0}^{*}$ is conservative and preserves Tot), we conclude that the above totalization is indeed trivial, and thus $k$-forms satisfy the asserted descent.

Now it remains to consider the full completed algebra of forms. We have seen that for each $k \geq 0$, we have the asserted descent for $k$-forms. Thus it suffices to check that the totalization is complete with respect to $k$. But by construction, each $U_{n}$ is an affine derived scheme, and hence the algebra of differential forms on $U_{n}$ is complete with respect to $k$. This immediately establishes the assertion and concludes the proof. 
Before continuing to a similar statement for sheaves, we record an interesting independent consequence of the above proposition. Recall that for a quasi-compact derived scheme $X$ with affine diagonal, we have established a functorial equivalence between Hochschild chains and differential forms

$$
H C\left(\mathcal{O}_{X}\right) \simeq \operatorname{Sym}^{\bullet}\left(\Omega_{X}[1]\right) .
$$

Recall as well that the algebra of differential forms on $X$ is connective, in particular complete: the natural map between differential forms and their completion is an equivalence

$$
\operatorname{Sym}^{\bullet}\left(\Omega_{X}[-1]\right) \stackrel{\sim}{\longrightarrow} \widehat{\operatorname{Sym}} \bullet\left(\Omega_{X}[-1]\right) .
$$

Corollary 6.5. For a quasi-compact derived scheme $X$ with affine diagonal, the assignment of Hochschild chains

$$
U \longmapsto H C\left(\mathcal{O}_{U}\right)=\mathcal{O}_{U} \otimes_{\mathcal{O}_{U} \otimes \mathcal{O}_{U}} \mathcal{O}_{U}
$$

forms a sheaf on the smooth site of $X$.

In the next section, we will explain the relationship between the formal loop space and formal odd tangent bundle in a global setting.

The main aim of this section is the following descent for quasicoherent sheaves.

Theorem 6.6. For a geometric stack $X$ and smooth maps $U \rightarrow X$, the assignment of $\mathbb{G}_{m^{-}}$ equivariant quasicoherent sheaves

$$
U \longmapsto \mathrm{QC}\left(\widehat{\mathbb{T}}_{U}[-1]\right)^{\mathbb{G}_{m}}
$$

forms a sheaf of stable $\infty$-categories with $B \mathbb{G}_{a}$-action on the smooth site of $X$. In particular, the assignment of $B \mathbb{G}_{a} \rtimes \mathbb{G}_{m}$-equivariant sheaves also forms a sheaf.

Proof. All of our arguments will be manifestly $B \mathbb{G}_{a}$-equivariant. Fix a smooth affine cover $u: U \rightarrow X$ with corresponding augmented Cech simplicial affine derived scheme $U \bullet \bullet X$. It suffices to show that pullback induces an equivalence

$$
\mathrm{QC}\left(\widehat{\mathbb{T}}_{X}[-1]\right)^{\mathbb{G}_{m}} \stackrel{\sim}{\longrightarrow} \operatorname{Tot}\left[\mathrm{QC}\left(\widehat{\mathbb{T}}_{U \cdot}[-1]\right)^{\mathbb{G}_{m}}\right] .
$$

For $k \geq 0$, let $\mathbf{S}_{X}^{\leq k}=\operatorname{Sym}^{\bullet} \Omega_{X}[1] / \operatorname{Sym}^{>k} \Omega_{X}[1]$ be the natural graded quotient algebra. Observe that the left hand side of the above asserted equivalence can be calculated by the limit of modules

$$
\mathrm{QC}\left(\widehat{\mathbb{T}}_{X}[-1]\right)^{\mathbb{G}_{m}} \stackrel{\sim}{\longrightarrow} \lim _{k}\left(\mathbf{S}_{X}^{\leq k}-\bmod _{\mathbf{z}}\right) .
$$

Similarly, the right hand side is the totalization of the limits of graded modules

$$
\mathrm{QC}\left(\widehat{\mathbb{T}}_{U}[-1]\right)^{\mathbb{G}_{m}} \stackrel{\sim}{\longrightarrow} \lim _{k}\left(\mathbf{S}_{U_{\bullet}}^{\leq k}-\bmod _{\mathbf{z}}\right) .
$$

Now by the previous proposition, we have equivalences

$$
\mathbf{S}_{X}^{\leq k}-\bmod _{\mathbf{Z}} \stackrel{\sim}{\longrightarrow} \operatorname{Tot}\left[\mathbf{S}_{U}^{\leq k}-\bmod _{\mathbf{z}}\right]
$$

Since limits commute with limits, we obtain the expression

$$
\mathrm{QC}\left(\widehat{\mathbb{T}}_{X}[-1]\right)^{\mathbb{G}_{m}} \stackrel{\sim}{\longrightarrow} \operatorname{Tot}\left[\lim _{k}\left(\mathbf{S}_{U_{\bullet}}^{\leq k} \bmod _{\mathbf{z}}\right)\right]
$$

But we also have the standard identity

$$
\mathrm{QC}\left(\widehat{\mathbb{T}}_{U}[-1]\right)^{\mathbb{G}_{m}} \simeq \widehat{\operatorname{Sym}}_{U_{\bullet}}^{\bullet}-\operatorname{cmod}_{\mathbf{z}} \simeq \lim _{k}\left(\mathbf{S}_{U}^{\leq k} \bmod _{\mathbf{z}}\right) .
$$

Assembling the above equivalences completes the proof of the theorem. 
6.3. Formal loops and odd tangents. In this section, we will compare the formal loop space and formal odd tangent bundle of a geometric stack. To begin, we will compare the loop space and odd tangent bundle infinitesimally.

6.3.1. Infinitesimal identification. Recall that we have a canonical projection and section

$$
\pi: \mathcal{L} X \longleftrightarrow X: u
$$

where $\pi$ is evaluation at the unit $e \in S^{1}$, and $u$ is the inclusion of constant loops.

By the cotangent complex to $\pi: \mathcal{L} X \rightarrow X$ along the section $u: X \rightarrow \mathcal{L} X$, we mean the pullback $u^{*} \Omega_{\mathcal{L} X} \in \mathrm{QC}(X)$.

Lemma 6.7. Let $X$ be a geometric stack. The cotangent complex to $\pi: \mathcal{L} X \rightarrow X$ along the section $u: X \rightarrow \mathcal{L} X$ of constant loops is functorially equivalent to the direct sum $\Omega_{X} \oplus \Omega_{X}[1]$, and the relative cotangent complex of $\pi$ along $u$ is functorially equivalent to the shifted cotangent complex $\Omega_{X}[1]$.

Proof. By definition, the loop space $\mathcal{L} X$ is the intersection

$$
\mathcal{L} X=X \times_{X \times X} X
$$

of the diagonal $\Delta: X \rightarrow X \times X$ with itself. Therefore there is a natural Mayer-Vietoris distinguished triangle

$$
\pi^{*} \Delta^{*} \Omega_{X \times X} \longrightarrow \pi^{*} \Omega_{X} \oplus \pi^{*} \Omega_{X} \longrightarrow \Omega_{\mathcal{L} X}
$$

of sheaves on $\mathcal{L} X$. Our convention is that the first map is induced by restricting a one-form on $X \times X$ to the diagonal $X$, then mapping it to the corresponding anti-diagonal one-form in the direct sum.

Pulling back along the unit section $u$, and applying the identity $\pi \circ u=\operatorname{id}_{X}$, gives a distinguished triangle

$$
\Omega_{X} \oplus \Omega_{X} \simeq \Delta^{*} \Omega_{X \times X} \longrightarrow \Omega_{X} \oplus \Omega_{X} \longrightarrow u^{*} \Omega_{\mathcal{L} X}
$$

of sheaves on $X$. Under our conventions, the first map is nothing more than the map of oneforms

$$
\left(\omega_{1}, \omega_{2}\right) \mapsto\left(\omega_{1}+\omega_{2},-\left(\omega_{1}+\omega_{2}\right)\right) .
$$

Thus we conclude that $u^{*} \Omega_{\mathcal{L} X} \simeq \Omega_{X} \oplus \Omega_{X}[1]$, and the relative part is the summand $\Omega_{X}[1]$.

Remark 6.8. For a perhaps more conceptual alternative proof, one can identify the relative cotangent complex as the stabilization functor from affine schemes over $X$ to quasicoherent sheaves on $X$. This implies that it intertwines the based loops functor $\Omega$ with the shift functor [1]. Hence one can identify the relative cotangent complex to the free loop space of $X$ with the shifted cotangent complex $\Omega_{X}[1]$.

6.3.2. The derived exponential map. Now we arrive at the main construction of this section.

Let $X$ be a geometric stack. Fix a smooth affine cover $U \rightarrow X$ with corresponding augmented Cech simplicial affine derived scheme $U_{\bullet} \rightarrow X$. Taking formal loops, we obtain a corresponding augmented simplicial affine derived scheme $\widehat{\mathcal{L}} U \bullet \rightarrow \widehat{\mathcal{L}} X$. While it is not true that $\widehat{\mathcal{L}} X$ is the geometric realization of $\widehat{\mathcal{L}} U_{\bullet}$, we will prove that $\mathcal{O}_{\widehat{\mathcal{L}} X}$ is the totalization of the corresponding cosimplicial complete algebra $\mathcal{O}_{\widehat{\mathcal{L}} U}$. To achieve this, we will compare with formal odd tangent bundles where we have seen such descent holds.

Working relative to $X$, we have a morphism of complete $\mathcal{O}_{X}$-algebras

$$
\mathcal{O}_{\widehat{\mathcal{L}} X} \longrightarrow \operatorname{Tot}\left[\mathcal{O}_{\widehat{\mathcal{L}} U_{\bullet}}\right] \simeq \operatorname{Tot}\left[\mathcal{O}_{\widehat{\mathbb{T}}_{U}}\right] \simeq \mathcal{O}_{\widehat{\mathbb{T}}_{X}[-1]} .
$$


Here the first equivalence is the identification of formal loop spaces and formal odd tangent bundles for affine schemes; the second equivalence is the assertion of Proposition 6.4. Passing to formal spectra relative to $X$, we obtain a morphism of derived stacks

$$
\exp : \widehat{\mathbb{T}}_{X}[-1] \longrightarrow \widehat{\mathcal{L}} X \text {. }
$$

Since all of the above constructions are equivariant, exp intertwines the translation $B \mathbb{G}_{a}$-action on $\widehat{\mathbb{T}}_{X}[-1]$ with the loop rotation $S^{1}$-action on $\widehat{\mathcal{L}} X$.

Let us write $\widehat{\mathcal{L}}^{u} X$ for the formal completion of unipotent loops $\mathcal{L}^{u} X$ along constant loops. Since all loops into affines are unipotent, we find by construction that exp factors through the formal completion of unipotent loops

$$
\exp : \widehat{\mathbb{T}}_{X}[-1] \longrightarrow \widehat{\mathcal{L}}^{u} X \longrightarrow \widehat{\mathcal{L}} X .
$$

Observe as above that as a map to the formal unipotent loop space, exp is $\mathbb{G}_{m}$-equivariant.

Recall that the canonical $\mathbb{G}_{m}$-action on the affinization $\operatorname{Aff}\left(S^{1}\right) \simeq B \mathbb{G}_{a}$ induces one on the unipotent loop space $\mathcal{L}^{u} X=\operatorname{Map}\left(B \mathbb{G}_{a}, X\right)$. We also have a canonical $\mathbb{G}_{m}$-action on the odd tangent bundle $\mathbb{T}_{X}[-1]$ which descends to the formal odd tangent bundle $\widehat{\mathbb{T}}_{X}[-1]$.

Theorem 6.9. For $X$ a geometric stack, the exponential map

$$
\exp : \widehat{\mathbb{T}}_{X}[-1] \longrightarrow \widehat{\mathcal{L}} X \text {. }
$$

is an equivalence.

Proof. Let us first deduce the theorem from the claim that the relative cotangent complex of $\exp : \widehat{\mathbb{T}}_{X}[-1] \rightarrow \widehat{\mathcal{L}} X$ vanishes. By filtering by powers of the augmentation ideals, we may write the complete $\mathcal{O}_{X}$-algebras $\mathcal{O}_{\widehat{\mathcal{L}} X}$ and $\mathcal{O}_{\widehat{\mathbb{T}}_{X}[-1]}$ as inverse limits of a sequence of square-zero extensions. The morphism exp automatically preserves this filtration. At each step we apply the result of [L2, 7.4.2] (see also [HAG2, 1.4.2]) asserting that the cotangent complex controls square zero extensions. (More specifically, the obstruction to lifting a homomorphism to a square-zero extension and the space of lifts are both described by shifted homomorphisms out of the relative cotangent complex, see [L2, 7.4.2.3].) Thus we deduce inductively that exp is an equivalence.

We will now establish the vanishing of the relative cotangent complex of the exponential map.

First, we claim that the restriction of the relative cotangent complex of exp along the zero section $z: X \rightarrow \widehat{\mathbb{T}}_{X}[-1]$ vanishes. To see this, note that Lemma 6.7 is functorial and so by Proposition 6.4 compatible with descent. Thus the identification of Lemma 6.7 agrees with the linearization of exp.

Next, we claim an easy version of Nakayama's lemma holds: if $\mathcal{M} \in \mathrm{QC}\left(\widehat{\mathbb{T}}_{X}[1]\right)$ satisfies $z^{*} \mathcal{M} \simeq 0$, then in fact $\mathcal{M} \simeq 0$. We include the proof for the reader's convenience. For each $k \geq 0$, consider the morphism

$$
z_{k}: \mathbb{T}_{\bar{X}}^{\leq k}[1]=\operatorname{Spec}_{\mathcal{O}_{X}}\left(\operatorname{Sym}^{\bullet}\left(\Omega_{X}[1]\right) / \operatorname{Sym}^{>k}\left(\Omega_{X}[1]\right)\right) \longrightarrow \widehat{\mathbb{T}}_{X}[1] .
$$

So in particular, for $k=0$, we have $\mathbb{T}_{\bar{X}}^{\leq 0}[1]=X$ and $z_{0}=z$. To prove the claim, it suffices to show that $z_{k}^{*} \mathcal{M} \simeq 0$, for all $k \geq 0$.

We proceed by induction. For $k=0$, we have seen $z_{0}^{*} \mathcal{M} \simeq 0$. Now for any $k \geq 0$, inside of $\mathrm{QC}\left(\widehat{\mathbb{T}}_{X}[1]\right)$, we have a distinguished triangle of modules

$$
z_{*} \operatorname{Sym}^{k+1}\left(\Omega_{X}[1]\right) \longrightarrow \mathcal{O}_{\mathbb{T}_{\bar{X}}^{\leq k+1}[1]}^{\longrightarrow} \mathcal{O}_{\mathbb{T}_{\bar{X}}^{\leq k}}[1]
$$


By the projection formula, we have the vanishing

$$
z_{*} \operatorname{Sym}^{k+1}\left(\Omega_{X}[1]\right) \otimes_{\mathcal{O}_{\widehat{\mathbb{T}}_{X}[1]}} \mathcal{M} \simeq z_{*}\left(\operatorname{Sym}^{k+1}\left(\Omega_{X}[1]\right) \otimes_{\mathcal{O}_{X}} z^{*} \mathcal{M}\right) \simeq 0
$$

and by induction, we have the vanishing

$$
z_{k}^{*} \mathcal{M}=\mathcal{O}_{\mathbb{T}_{X}^{\leq k}[1]} \otimes_{\mathcal{O}_{\mathbb{T}_{X}[1]}} \mathcal{M} \simeq 0
$$

Thus we also have the vanishing

$$
z_{k+1}^{*} \mathcal{M}=\mathcal{O}_{\mathbb{T}_{X}^{\leq k+1}[1]} \otimes_{\mathcal{O}_{\widehat{\mathbb{T}}_{X}[1]}} \mathcal{M} \simeq 0 .
$$

This completes the proof of the vanishing of relative cotangents, and hence of the theorem.

Corollary 6.10. For $X$ a geometric stack, formal loops are unipotent: the canonical map $\widehat{\mathcal{L}} X \rightarrow \mathcal{L} X$ factors through the canonical map $\mathcal{L}^{u} X \rightarrow \mathcal{L} X$. Moreover, the composite map

$$
\widehat{\mathbb{T}}_{X}[-1] \stackrel{\exp }{\longrightarrow} \widehat{\mathcal{L}} X \longrightarrow \mathcal{L}^{u} X
$$

is canonically $B \mathbb{G}_{a} \rtimes \mathbb{G}_{m}$-equivariant.

Remark 6.11 (Unipotent loops embed in odd tangents). Consider the deformation to the normal cone $\mathbb{T}_{X}[-1]$ of the loop space $\mathcal{L} X$ along the constant loops $X$. The $\mathbb{G}_{m}$-action on the unipotent loop space $\mathcal{L}^{u} X$ trivializes the restriction of this deformation to unipotent loops. In this way, we obtain a canonical embedding

$$
\mathcal{L}^{u} X \longrightarrow \mathbb{T}_{X}[-1]
$$

Taking formal completions along constant loops, and using Theorem 6.9 to identify the formal loop space $\widehat{\mathcal{L}} X$ with the formal unipotent loop space $\widehat{\mathcal{L}}^{u} X$, we obtain from the above morphism the inverse to exp.

6.4. Equivariant sheaves on formal loop spaces. Our locality results for formal odd tangent bundles together with their identifications with formal loop spaces now allow us to immediately transport all of our local results to formal loop spaces of smooth geometric underived stacks.

Combining Theorems 6.6 and 6.9, we obtain the following corollary. It can be viewed as a linear version of locality for formal loops.

Corollary 6.12. For a geometric stack $X$ and smooth maps $U \rightarrow X$, the assignment of $S^{1}$ equivariant quasicoherent sheaves

$$
U \longmapsto \mathrm{QC}(\widehat{\mathcal{L}} U)^{S^{1}}
$$

forms a sheaf on the smooth site of $X$.

Recall that for $X$ a derived scheme, we write $\Omega_{X}^{-\bullet}[d]$ for its extended de Rham algebra, and $\Omega_{X, d}^{-\bullet}$ for its de Rham complex. Since both form sheaves of $k$-algebras in the smooth topology, it makes sense to consider them for a geometric stack. Finally, note as well that the identification $\widehat{\mathcal{L}} X \simeq \widehat{\mathbb{T}}_{X}[-1]$ for a geometric stack $X$ allows us to consider $B \mathbb{G}_{a} \rtimes \mathbb{G}_{m}$-equivariant sheaves.

Theorem 6.13. For $X$ a smooth underived geometric stack, there are canonical equivalences of stable $\infty$-categories

$$
\begin{gathered}
\mathrm{QC}(\widehat{\mathcal{L}} X)^{B \mathbb{G}_{a} \rtimes \mathbb{G}_{m}} \simeq \Omega_{X}^{\bullet}[d]-\bmod _{\mathbf{z}} \\
\mathrm{QC}(\widehat{\mathcal{L}} X)_{\text {per }}^{B \mathbb{G}_{a} \rtimes \mathbb{G}_{m}} \simeq \Omega_{X, d^{\bullet}}^{\bullet} \text { mod }
\end{gathered}
$$

Similarly, we also have generalizations to geometric stacks of Koszul dual descriptions and further variations described for derived schemes. We leave the statements to the reader. 


\section{REFERENCES}

[BB] A. Beilinson and J. Bernstein, A proof of Jantzen conjectures. I. M. Gelfand Seminar, 1-50, Adv. Soviet Math., 16, Part 1, Amer. Math. Soc., Providence, RI, 1993.

[BD] A. Beilinson and V. Drinfeld, Quantization of Hitchin Hamiltonians and Hecke Eigensheaves. Preprint, available at math.uchicago.edu/ $\sim$ arinkin.

[BGS] A. Beilinson, V. Ginzburg and W. Soergel, Koszul duality patterns in representation theory. Jour. Amer. Math. Soc. 9 (1996), no. 2, 473-527.

[BFN] D. Ben-Zvi, J. Francis and D. Nadler, Integral transforms and Drinfeld centers in derived algebraic geometry. Jour. Amer. Math. Soc. 23 (2010), 909-966.

[BN1] D. Ben-Zvi and D. Nadler, Loop Spaces and Langlands Parameters. arXiv:0706.0322

[BN2] D. Ben-Zvi and D. Nadler, Loop Spaces and Representations. arXiv:1004.512.

[Ber] J. Bergner, A survey of $(\infty, 1)$-categories. arXiv:math/0610239

[BGT] A. Blumberg, D. Gepner, G. Tabuada, A universal characterization of higher algebraic $K$-theory, arXiv:1001.2282

[Co] A. Connes, Cohomologie cyclique et foncteurs Ext ${ }^{n}$. C. R. Acad. Sci. Paris Sr. I Math. 296 (1983), no. 23, 953-958.

[D] V. Drinfeld, DG quotients of DG categories. arXiv:math.KT/0210114. J. Algebra 272 (2004), no. 2, 643-691.

[DHK] W. Dwyer, M. Hopkins and D. Kan, The homotopy theory of cyclic sets. Trans. Amer. Math. Soc. 291 (1985), no. 1, 281-289.

[DK] W. Dwyer and D. Kan, Three homotopy theories for cyclic modules. Proceedings of the Northwestern conference on cohomology of groups (Evanston, Ill., 1985). J. Pure Appl. Algebra 44 (1987), no. 1-3, $165-175$.

[GKM] M. Goresky, R. Kottwitz and R. MacPherson, Equivariant cohomology, Koszul duality, and the localization theorem. Invent. Math. 131 (1998), no. 1, 25-83.

[HKR] G. Hochschild, B. Kostant, and A. Rosenberg, Differential forms on regular affine algebras. Trans. Amer. Math. Soc. 1021962 383-408.

[J] J.D.S. Jones, Cyclic homology and equivariant homology. Invent. Math. 87 (1987), no. 2, 403-423.

[Jo] A. Joyal, Quasi-categories and Kan complexes. J. Pure Appl. Algebra 175 (2002) 207-222.

[Ka] M. Kapranov, On DG-modules over the de Rham complex and the vanishing cycles functor. Algebraic geometry (Chicago, IL, 1989), 57-86, Lect. Notes in Math. 1479, Springer, Berlin, 1991.

[Kas] C. Kassel, Cyclic homology, comodules, and mixed complexes. J. Algebra 107 (1987), no. 1, $195-216$.

[Ke] B. Keller, On differential graded categories. arXiv:math.AG/0601185 International Congress of Mathematicians. Vol. II, 151-190, Eur. Math. Soc., Zürich, 2006.

[Lo] J.-L. Loday, Cyclic homology. Appendix E by María O. Ronco. Second edition. Chapter 13 by the author in collaboration with Teimuraz Pirashvili. Grundlehren der Mathematischen Wissenschaften [Fundamental Principles of Mathematical Sciences], 301. Springer-Verlag, Berlin, 1998.

[L1] J. Lurie, Higher topos theory. arXiv:math.CT/0608040 Annals of Mathematics Studies, 170. Princeton University Press, Princeton, NJ, 2009.

[L2] J. Lurie, Higher Algebra. Available at http://www.math.harvard.edu/ ${ }^{2}$ lurie/

[L3] J. Lurie, Derived Algebraic Geometry 7: Spectral Schemes. Available at http://www.math.harvard.edu/ ${ }^{\sim}$ lurie/

[L4] J. Lurie, Derived Algebraic Geometry 8: Quasi-coherent sheaves and Tannaka duality theorems. Available at http://www.math.harvard.edu/ lurie/

[P] L. Positselski, Nonhomogeneous quadratic duality and curvature. (Russian) Funktsional. Anal. i Prilozhen. 27 (1993), no. 3, 57-66, 96; translation in Funct. Anal. Appl. 27 (1993), no. 3, 197-204

[PP] A. Polishchuk and L. Positselski, Quadratic algebras. University Lecture Series, 37. American Mathematical Society, Providence, RI, 2005.

[S] C. Simpson, The Hodge filtration on nonabelian cohomology. Algebraic geometry—Santa Cruz 1995, 217-281, Proc. Sympos. Pure Math., 62, Part 2, Amer. Math. Soc., Providence, RI, 1997.

[ST] C. Simpson and C. Teleman, de Rham's theorem for $\infty$-stacks. Available at www.dpmms.cam.ac.uk/ ${ }^{\sim}$ teleman/math/simpson.pdf.

[St] J. Stasheff, $H$-spaces from a homotopy point of view. Lecture Notes in Mathematics, Vol. 161. SpringerVerlag, Berlin-New York 1970

[To1] B. Toën, Affine stacks (Champs affines). arXiv:math.AG/0012219 Champs affines. Selecta Math. (N.S.) 12 (2006), no. 1, 39-135. 
[To2] B. Toën, Higher and Derived Stacks: a global overview. e-print arXiv:math.AG/0604504 Algebraic geometry-Seattle 2005. Part 1, 435-487, Proc. Sympos. Pure Math., 80, Part 1, Amer. Math. Soc., 2009.

[HAG1] B. Toën and G. Vezzosi, Homotopical Algebraic Geometry I: Topos theory. Adv. Math. arXiv:math.AG/0404373

[HAG2] B. Toën and G. Vezzosi, Homotopical Algebraic Geometry II: geometric stacks and applications. Memoirs of the AMS. arXiv:math.AG/0404373

[ToVe3] B. Toën and G. Vezzosi, A note on Chern character, loop spaces and derived algebraic geometry. Preprint arXiv:0804.1274

[ToVe4] B. Toën and G. Vezzosi, Infinies-categories monoidales rigides, traces et caracteres de Chern. Preprint arXiv:0903.3292

[ToVe5] B. Toën and G. Vezzosi, $S^{1}$-Equivariant simplicial algebras and de Rham theory. Preprint arXiv:0904.3256

[W] C. Weibel, The Hodge filtration and cyclic homology. $K$-Theory 12 (1997), no. 2, 145-164.

[WG] C. Weibel and S. Geller, Étale descent for Hochschild and cyclic homology. Comment. Math. Helv. 66 (1991), no. 3, 368-388.

Department of Mathematics, University of Texas, Austin, TX 78712-0257

E-mail address: benzvi@math.utexas.edu

Department of Mathematics, Northwestern University, Evanston, IL 60208-2370

E-mail address: nadler@math.northwestern.edu 